\title{
Multi-gap Resistive Plate Chambers as a Time-of-Flight System for the PHENIX Experiment
}

\author{
Julia Velkovska \\ Department of Physics and Astronomy \\ Vanderbilt University
}

\section{Closeout report on Grant DE-FG02-04ER41333}

Department of Energy Outstanding Junior Investigator Award

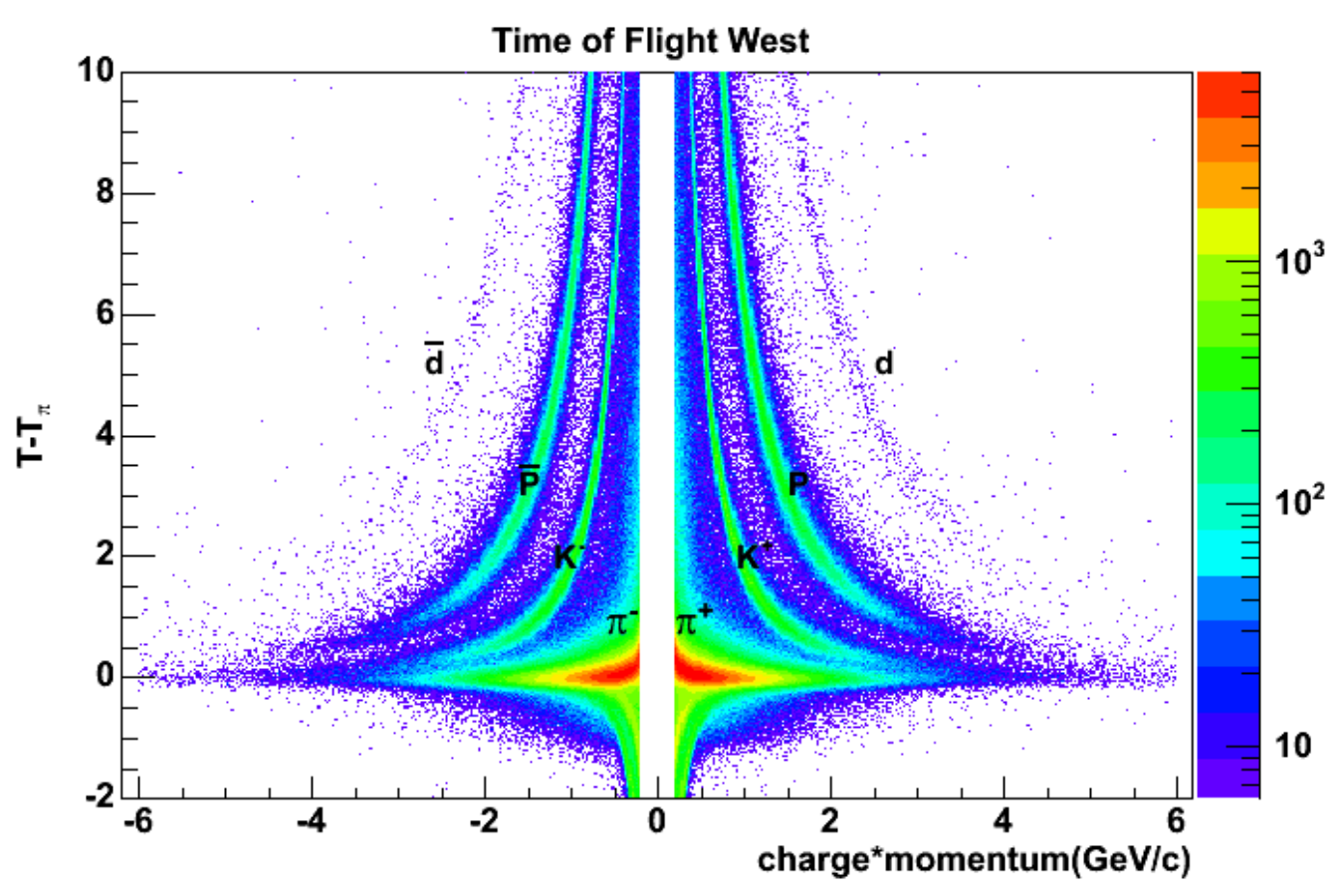




\section{Project Overview}

One of the early and striking experimental discoveries at RHIC is the large baryon and anti-baryon enhancement over the expectation from parton fragmentation. This phenomenon is still not completely understood, although there are indications that the baryon anomaly is due to quark recombination in the dense medium. It is also possible that the fragmentation process itself is modified by the medium in a way which favors baryon production. In particular, differences in formation time for baryons and mesons could lead to different suppression in the medium. Exotic mechanisms for baryon transport through gluon junction have also been proposed, although they are very difficult to test experimentally. Particle identification (PID) in a broad momentum range is necessary to test the various theoretical predictions.

Supported by this OJI grant and additional DOE funds, the PI of this grant, Prof. Julia Velkovska, designed and built a high resolution time-of-flight system for the PHENIX experiment at RHIC. The project was based on multi-gap resistive plate chambers, which proved both cost-effective and reliable. The detector was installed in the PHENIX West arm spectrometer during the summer of 2006 and has been successfully operated for the last 7 years. The TOF.West detector performs extremely well, exceeding the design goals for timing resolution. It is expected that the detector will continue to be in operation until the PHENIX detector will transition to SPHENIX in several years from now.

\section{Sicentific accomplishments}

So far, the project has yielded 2 Ph.D. theses (Dr. Hugo Valle, Dr. Ronald Belmont), one M.S. thesis (Brian Love) completed at Vanderbilt University and several thesis at other universities that participate in the PHENIX collaboration. There are many PHENIX physics results that used the TOF.West detector to perform particle identification. In the next section, the full text of two recent publications that resulted from this project is included, in lieu of a scientific report. The full text of the associated theses can be found on the PHENIX

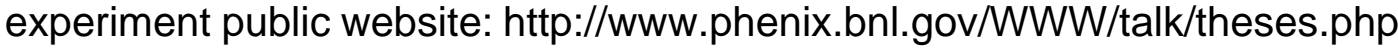
and direct hyperlinks are included below.

1. Ronald Belmont, "Measurements of identified hadrons in $A u+A u$ and $d+A u$ collisions at $\sqrt{ } \mathrm{s}_{\mathrm{NN}}=200 \mathrm{GeV}$ ", Ph.D. 2012

2. Hugo Valle, "Source dynamics from Deuteron and Anti-deuteron Production in $\sqrt{ }_{\mathrm{S}_{\mathrm{NN}}}=200 \mathrm{GeV}$ Au+Au Collisions", Ph.D. 2008

3. Brian Love, "The Design, Implementation and Performance of the PHENIX Time-of-Flight West Detector", M.S. 2009 
published in Phys. Rev. C 85, 064914 (2012)

\section{Deviation from quark number scaling of the anisotropy parameter $v_{2}$ of pions, kaons, and protons in $\mathrm{Au}+\mathrm{Au}$ collisions at $\sqrt{s_{N N}}=200 \mathrm{GeV}$}

A. Adare, ${ }^{11}$ S. Afanasiev,${ }^{27}$ C. Aidala, ${ }^{39}$ N.N. Ajitanand, ${ }^{56}$ Y. Akiba, ${ }^{50,51}$ H. Al-Bataineh, ${ }^{45}$ J. Alexander, ${ }^{56}$ K. Aoki,${ }^{32,}{ }^{50}$ Y. Aramaki, ${ }^{10}$ E.T. Atomssa, ${ }^{33}$ R. Averbeck,,${ }^{57}$ T.C. Awes, ${ }^{46}$ B. Azmoun, ${ }^{5}$ V. Babintsev,${ }^{22}$ M. Bai, ${ }^{4}$ G. Baksay, ${ }^{18}$ L. Baksay, ${ }^{18}$ K.N. Barish, ${ }^{6}$ B. Bassalleck,,${ }^{44}$ A.T. Basye,${ }^{1}$ S. Bathe,${ }^{6}$ V. Baublis,${ }^{49}$ C. Baumann, ${ }^{40}$ A. Bazilevsky, ${ }^{5}$ S. Belikov, ${ }^{5}$, R. Belmont,${ }^{61}$ R. Bennett,${ }^{57}$ A. Berdnikov, ${ }^{53}$ Y. Berdnikov, ${ }^{53}$ A.A. Bickley, ${ }^{11}$ J.S. Bok, ${ }^{65}$ K. Boyle, ${ }^{57}$ M.L. Brooks,${ }^{35}$ H. Buesching, ${ }^{5}$ V. Bumazhnov, ${ }^{22}$ G. Bunce, ${ }^{5,51}$ S. Butsyk, ${ }^{35}$ C.M. Camacho, ${ }^{35}$ S. Campbell,,${ }^{57}$ C.-H. Chen, ${ }^{57}$ C.Y. Chi, ${ }^{12}$ M. Chiu, ${ }^{5}$ I.J. Choi, ${ }^{65}$ R.K. Choudhury ${ }^{3}$ P. Christiansen, ${ }^{37}$ T. Chujo, ${ }^{60}$ P. Chung, ${ }^{56}$ O. Chvala, ${ }^{6}$ V. Cianciolo, ${ }^{46}$ Z. Citron, ${ }^{57}$ B.A. Cole, ${ }^{12}$ M. Connors,${ }^{57}$ P. Constantin, ${ }^{35}$ M. Csanád, ${ }^{16}$ T. Csörgő, ${ }^{64}$ T. Dahms, ${ }^{57}$ S. Dairaku, ${ }^{32,50}$ I. Danchev, ${ }^{61}$ K. Das, ${ }^{19}$ A. Datta, ${ }^{39}$ G. David,${ }^{5}$ A. Denisov, ${ }^{22}$ A. Deshpande, ${ }^{51,57}$ E.J. Desmond,${ }^{5}$ O. Dietzsch, ${ }^{54}$ A. Dion, ${ }^{57}$ M. Donadelli, ${ }^{54}$ O. Drapier, ${ }^{33}$ A. Drees,${ }^{57}$ K.A. Drees,${ }^{4}$ J.M. Durham,${ }^{57}$ A. Durum,${ }^{22}$ D. Dutta,${ }^{3}$ S. Edwards,${ }^{19}$ Y.V. Efremenko, ${ }^{46}$ F. Ellinghaus, ${ }^{11}$ T. Engelmore,${ }^{12}$ A. Enokizono, ${ }^{34}$ H. En'yo,${ }^{50,51}$ S. Esumi,${ }^{60}$ B. Fadem, ${ }^{41}$ D.E. Fields, ${ }^{44}$ M. Finger, ${ }^{7}$ M. Finger, Jr.,${ }^{7}$ F. Fleuret,${ }^{33}$ S.L. Fokin, ${ }^{31}$ Z. Fraenkel, ${ }^{63}$, 团 J.E. Frantz,${ }^{57}$ A. Franz,${ }^{5}$ A.D. Frawley ${ }^{19}$ K. Fujiwara,${ }^{50}$ Y. Fukao, ${ }^{50}$ T. Fusayasu, ${ }^{43}$ I. Garishvili, ${ }^{58}$ A. Glenn, ${ }^{11}$ H. Gong, ${ }^{57}$ M. Gonin, ${ }^{33}$ Y. Goto, ${ }^{50,51}$ R. Granier de Cassagnac, ${ }^{33}$ N. Grau,${ }^{12}$ S.V. Greene, ${ }^{61}$ M. Grosse Perdekamp, ${ }^{23,}{ }^{51}$ T. Gunji, ${ }^{10}$ H.-Å. Gustafsson,, 37 , 田 J.S. Haggerty,${ }^{5}$ K.I. Hahn, ${ }^{17}$ H. Hamagaki, ${ }^{10}$ J. Hamblen, ${ }^{58}$ R. Han,${ }^{48}$ J. Hanks, ${ }^{12}$ E.P. Hartouni,${ }^{34}$ E. Haslum, ${ }^{37}$ R. Hayano, ${ }^{10}$ X. He ${ }^{20}$ M. Heffner, ${ }^{34}$ T.K. Hemmick, ${ }^{57}$ T. Hester, ${ }^{6}$ J.C. Hill,${ }^{26}$ M. Hohlmann, ${ }^{18}$ W. Holzmann, ${ }^{12}$ K. Homma, ${ }^{21}$ B. Hong,${ }^{30}$ T. Horaguchi, ${ }^{21}$ D. Hornback, ${ }^{58}$ S. Huang, ${ }^{61}$ T. Ichihara,${ }^{50,51}$ R. Ichimiya, ${ }^{50}$ J. Ide, ${ }^{41}$ Y. Ikeda,${ }^{60} \mathrm{~K}$. Imai, ${ }^{32,}{ }^{50} \mathrm{M}$. Inaba,${ }^{60} \mathrm{D}$. Isenhower, ${ }^{1} \mathrm{M}$. Ishihara, ${ }^{50} \mathrm{~T}$. Isobe,${ }^{10,}{ }^{50} \mathrm{M}$. Issah,${ }^{61} \mathrm{~A}$. Isupov, ${ }^{27}$

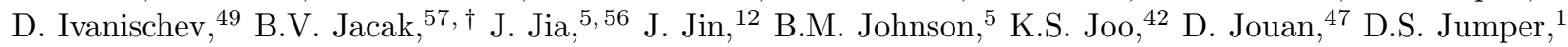
F. Kajihara, ${ }^{10}$ S. Kametani, ${ }^{50}$ N. Kamihara, ${ }^{51}$ J. Kamin,,${ }^{57}$ J.H. Kang,${ }^{65}$ J. Kapustinsky ${ }^{35}$ K. Karatsu, ${ }^{32,50}$ D. Kawall, ${ }^{39,51}$ M. Kawashima, ${ }^{50,52}$ A.V. Kazantsev, ${ }^{31}$ T. Kempel, ${ }^{26}$ A. Khanzadeev, ${ }^{49}$ K.M. Kijima, ${ }^{21}$ B.I. Kim, ${ }^{30}$ D.H. Kim, ${ }^{42}$ D.J. Kim, ${ }^{28}$ E. Kim,${ }^{55}$ E.J. Kim, ${ }^{8}$ S.H. Kim ${ }^{65}$ Y.J. Kim,${ }^{23}$ E. Kinney, ${ }^{11}$ K. Kiriluk, ${ }^{11}$ Á. Kiss, ${ }^{16}$ E. Kistenev ${ }^{5}$ L. Kochenda, ${ }^{49}$ B. Komkov ${ }^{49}$ M. Konno, ${ }^{60}$ J. Koster ${ }^{23}$ D. Kotchetkov, ${ }^{44}$ A. Kozlov ${ }^{63}$ A. Král, ${ }^{13}$

A. Kravitz, ${ }^{12}$ G.J. Kunde, ${ }^{35}$ K. Kurita, ${ }^{50,52}$ M. Kurosawa, ${ }^{50}$ Y. Kwon, ${ }^{65}$ G.S. Kyle, ${ }^{45}$ R. Lacey,${ }^{56}$ Y.S. Lai, ${ }^{12}$ J.G. Lajoie, ${ }^{26}$ A. Lebedev, ${ }^{26}$ D.M. Lee, ${ }^{35}$ J. Lee,${ }^{17}$ K. Lee ${ }^{55}$ K.B. Lee ${ }^{30}$ K.S. Lee,${ }^{30}$ M.J. Leitch, ${ }^{35}$ M.A.L. Leite, ${ }^{54}$

E. Leitner, ${ }^{61}$ B. Lenzi, ${ }^{54}$ X. Li,${ }^{9}$ P. Liebing, ${ }^{51}$ L.A. Linden Levy, ${ }^{11}$ T. Liška,${ }^{13}$ A. Litvinenko, ${ }^{27}$ H. Liu, ${ }^{35,45}$ M.X. Liu ${ }^{35}$ B. Love ${ }^{61}$ R. Luechtenborg, ${ }^{40}$ D. Lynch, ${ }^{5}$ C.F. Maguire ${ }^{61}$ Y.I. Makdisi, ${ }^{4}$ A. Malakhov ${ }^{27}$ M.D. Malik,${ }^{44}$ V.I. Manko, ${ }^{31}$ E. Mannel, ${ }^{12}$ Y. Mao,${ }^{48,50}$ H. Masui, ${ }^{60}$ F. Matathias, ${ }^{12}$ M. McCumber,${ }^{57}$ P.L. McGaughey, ${ }^{35}$ N. Means, ${ }^{57}$ B. Meredith, ${ }^{23}$ Y. Miake, ${ }^{60}$ A.C. Mignerey, ${ }^{38}$ P. Mikeš,${ }^{7}{ }^{25}$ K. Miki,${ }^{50,60}$ A. Milov ${ }^{5}$ M. Mishra, ${ }^{2}$ J.T. Mitchell,${ }^{5}$ A.K. Mohanty ${ }^{3}$ Y. Morino, ${ }^{10}$ A. Morreale, ${ }^{6}$ D.P. Morrison, ${ }^{5}$ T.V. Moukhanova,${ }^{31}$ J. Murata $,{ }^{50,}, 52$ S. Nagamiya, ${ }^{29}$ J.L. Nagle, ${ }^{11}$ M. Naglis, ${ }^{63}$ M.I. Nagy, ${ }^{16}$ I. Nakagawa, ${ }^{50,51}$ Y. Nakamiya, ${ }^{21}$ T. Nakamura, ${ }^{21,}{ }^{29}$ K. Nakano, ${ }^{50,59}$ J. Newby, ${ }^{34}$ M. Nguyen, ${ }^{57}$ R. Nouicer, ${ }^{5}$ A.S. Nyanin, ${ }^{31}$ E. O'Brien, ${ }^{5}$ S.X. Oda, ${ }^{10}$ C.A. Ogilvie ${ }^{26}$ M. Oka, ${ }^{60}$ K. Okada,${ }^{51}$ Y. Onuki,${ }^{50}$ A. Oskarsson,,${ }^{37}$ M. Ouchida,${ }^{21,}{ }^{50}$ K. Ozawa, ${ }^{10}$ R. Pak, ${ }^{5}$ V. Pantuev, ${ }^{24,57}$

V. Papavassiliou, ${ }^{45}$ I.H. Park, ${ }^{17}$ J. Park,${ }^{55}$ S.K. Park,${ }^{30}$ W.J. Park,${ }^{30}$ S.F. Pate, ${ }^{45}$ H. Pei, ${ }^{26}$ J.-C. Peng, ${ }^{23}$ H. Pereira, ${ }^{14}$ V. Peresedov ${ }^{27}$ D.Yu. Peressounko, ${ }^{31}$ C. Pinkenburg, ${ }^{5}$ R.P. Pisani, ${ }^{5}$ M. Proissl,${ }^{57}$ M.L. Purschke, ${ }^{5}$ A.K. Purwar, ${ }^{35} \mathrm{H}$. Qu ${ }^{20}$ J. Rak, ${ }^{28}$ A. Rakotozafindrabe, ${ }^{33}$ I. Ravinovich, ${ }^{63}$ K.F. Read, ${ }^{46,}{ }^{58} \mathrm{~K}$. Reygers, ${ }^{40}$ V. Riabov, ${ }^{49}$ Y. Riabov, ${ }^{49}$ E. Richardson, ${ }^{38}$ D. Roach ${ }^{61}$ G. Roche ${ }^{36}$ S.D. Rolnick ${ }^{6}$ M. Rosati ${ }^{26}$ C.A. Rosen, ${ }^{11}$ S.S.E. Rosendahl,${ }^{37}$ P. Rosnet,${ }^{36}$ P. Rukoyatkin, ${ }^{27}$ P. Ružička, ${ }^{25}$ B. Sahlmueller ${ }^{40}$ N. Saito, ${ }^{29}$ T. Sakaguchi, ${ }^{5}$ K. Sakashita, ${ }^{50,59}$ V. Samsonov ${ }^{49}$ S. Sano, ${ }^{10,62}$ T. Sato,${ }^{60}$ S. Sawada, ${ }^{29}$ K. Sedgwick, ${ }^{6}$ J. Seele, ${ }^{11}$ R. Seidl,${ }^{23}$

A.Yu. Semenov, ${ }^{26}$ R. Seto, ${ }^{6}$ D. Sharma,${ }^{63}$ I. Shein, ${ }^{22}$ T.-A. Shibata, ${ }^{50,}{ }^{59}$ K. Shigaki, ${ }^{21}$ M. Shimomura, ${ }^{60}$ K. Shoji,${ }^{32,}{ }^{50}$ P. Shukla,${ }^{3}$ A. Sickles, ${ }^{5}$ C.L. Silva,${ }^{54}$ D. Silvermyr, ${ }^{46}$ C. Silvestre, ${ }^{14}$ K.S. Sim,${ }^{30}$ B.K. Singh, ${ }^{2}$ C.P. Singh, ${ }^{2}$ V. Singh, ${ }^{2}$ M. Slunečka, ${ }^{7}$ R.A. Soltz, ${ }^{34}$ W.E. Sondheim, ${ }^{35}$ S.P. Sorensen, ${ }^{58}$ I.V. Sourikova, ${ }^{5}$

N.A. Sparks, ${ }^{1}$ P.W. Stankus, ${ }^{46}$ E. Stenlund, ${ }^{37}$ S.P. Stoll,${ }^{5}$ T. Sugitate,${ }^{21}$ A. Sukhanov, ${ }^{5}$ J. Sziklai,${ }^{64}$ E.M. Takagui, ${ }^{54}$ A. Taketani, ${ }^{50,51}$ R. Tanabe ${ }^{60}$ Y. Tanaka,${ }^{43}$ K. Tanida,${ }^{32,50,51}$ M.J. Tannenbaum, ${ }^{5}$ S. Tarafdar,${ }^{2}$

A. Taranenko, ${ }^{56}$ P. Tarján, ${ }^{15}$ H. Themann, ${ }^{57}$ T.L. Thomas, ${ }^{44}$ M. Togawa,${ }^{32,50}$ A. Toia, ${ }^{57}$ L. Tomášek, ${ }^{25}$

H. Torii, ${ }^{21}$ R.S. Towell, ${ }^{1}$ I. Tserruya,${ }^{63}$ Y. Tsuchimoto, ${ }^{21}$ C. Vale,${ }^{5}{ }^{26}$ H. Valle,${ }^{61}$ H.W. van Hecke,${ }^{35}$

E. Vazquez-Zambrano, ${ }^{12}$ A. Veicht,${ }^{23}$ J. Velkovska,${ }^{61}$ R. Vértesi,${ }^{15,64}$ A.A. Vinogradov,${ }^{31}$ M. Virius,${ }^{13}$ V. Vrba ${ }^{25}$ E. Vznuzdaev ${ }^{49}$ X.R. Wang ${ }^{45}$ D. Watanabe, ${ }^{21}$ K. Watanabe,${ }^{60}$ Y. Watanabe,${ }^{50,51}$ F. Wei, ${ }^{26}$ R. Wei ${ }^{56}$ J. Wessels, ${ }^{40}$ S.N. White, ${ }^{5}$ D. Winter,${ }^{12}$ J.P. Wood,${ }^{1}$ C.L. Woody,${ }^{5}$ R.M. Wright,${ }^{1}$ M. Wysocki, ${ }^{11}$ W. Xie, ${ }^{51}$ Y.L. Yamaguchi, ${ }^{10}$ K. Yamaura,${ }^{21}$ R. Yang, ${ }^{23}$ A. Yanovich, ${ }^{22}$ J. Ying, ${ }^{20}$ S. Yokkaichi, ${ }^{50,}{ }^{51}$ Z. You, ${ }^{48}$ 
G.R. Young, ${ }^{46}$ I. Younus, ${ }^{44}$ I.E. Yushmanov, ${ }^{31}$ W.A. Zajc ${ }^{12}$ C. Zhang,${ }^{46}$ S. Zhou, ${ }^{9}$ and L. Zolin ${ }^{27}$

(PHENIX Collaboration)

${ }^{1}$ Abilene Christian University, Abilene, Texas 79699, USA

${ }^{2}$ Department of Physics, Banaras Hindu University, Varanasi 221005, India

${ }^{3}$ Bhabha Atomic Research Centre, Bombay 400 085, India

${ }^{4}$ Collider-Accelerator Department, Brookhaven National Laboratory, Upton, New York 11973-5000, USA

${ }^{5}$ Physics Department, Brookhaven National Laboratory, Upton, New York 11973-5000, USA

${ }^{6}$ University of California - Riverside, Riverside, California 92521, USA

${ }^{7}$ Charles University, Ovocný trh 5, Praha 1, 116 36, Prague, Czech Republic

${ }^{8}$ Chonbuk National University, Jeonju, 561-756, Korea

${ }^{9}$ Science and Technology on Nuclear Data Laboratory, China Institute of Atomic Energy, Beijing 102413, P. R. China

${ }^{10}$ Center for Nuclear Study, Graduate School of Science, University of Tokyo, 7-3-1 Hongo, Bunkyo, Tokyo 113-0033, Japan

${ }^{11}$ University of Colorado, Boulder, Colorado 80309, USA

${ }^{12}$ Columbia University, New York, New York 10027 and Nevis Laboratories, Irvington, New York 10533, USA

${ }^{13}$ Czech Technical University, Zikova 4, 16636 Prague 6, Czech Republic

${ }^{14}$ Dapnia, CEA Saclay, F-91191, Gif-sur-Yvette, France

${ }^{15}$ Debrecen University, H-4010 Debrecen, Egyetem tér 1, Hungary

${ }^{16}$ ELTE, Eötvös Loránd University, H - 1117 Budapest, Pázmány P. s. 1/A, Hungary

${ }^{17}$ Ewha Womans University, Seoul 120-750, Korea

${ }^{18}$ Florida Institute of Technology, Melbourne, Florida 32901, USA

${ }^{19}$ Florida State University, Tallahassee, Florida 32306, USA

${ }^{20}$ Georgia State University, Atlanta, Georgia 30303, USA

${ }^{21}$ Hiroshima University, Kagamiyama, Higashi-Hiroshima 739-8526, Japan

${ }^{22}$ IHEP Protvino, State Research Center of Russian Federation, Institute for High Energy Physics, Protvino, 142281, Russia

${ }^{23}$ University of Illinois at Urbana-Champaign, Urbana, Illinois 61801, USA

${ }^{24}$ Institute for Nuclear Research of the Russian Academy of Sciences, prospekt 60-letiya Oktyabrya 7a, Moscow 117312, Russia

${ }^{25}$ Institute of Physics, Academy of Sciences of the Czech Republic, Na Slovance 2, 182 21 Prague 8, Czech Republic

${ }^{26}$ Iowa State University, Ames, Iowa 50011, USA

${ }^{27}$ Joint Institute for Nuclear Research, 141980 Dubna, Moscow Region, Russia

${ }^{28}$ Helsinki Institute of Physics and University of Jyväskylä, P.O.Box 35, FI-40014 Jyväskylä, Finland

${ }^{29}$ KEK, High Energy Accelerator Research Organization, Tsukuba, Ibaraki 305-0801, Japan

${ }^{30}$ Korea University, Seoul, 136-701, Korea

${ }^{31}$ Russian Research Center "Kurchatov Institute", Moscow, 123098 Russia

${ }^{32}$ Kyoto University, Kyoto 606-8502, Japan

${ }^{33}$ Laboratoire Leprince-Ringuet, Ecole Polytechnique, CNRS-IN2P3, Route de Saclay, F-91128, Palaiseau, France

${ }^{34}$ Lawrence Livermore National Laboratory, Livermore, California 94550, USA

${ }^{35}$ Los Alamos National Laboratory, Los Alamos, New Mexico 87545, USA

${ }^{36}$ LPC, Université Blaise Pascal, CNRS-IN2P3, Clermont-Fd, 63177 Aubiere Cedex, France

${ }^{37}$ Department of Physics, Lund University, Box 118, SE-221 00 Lund, Sweden

${ }^{38}$ University of Maryland, College Park, Maryland 20742, USA

${ }^{39}$ Department of Physics, University of Massachusetts, Amherst, Massachusetts 01003-9337, USA

${ }^{40}$ Institut fur Kernphysik, University of Muenster, D-48149 Muenster, Germany

${ }^{41}$ Muhlenberg College, Allentown, Pennsylvania 18104-5586, USA

${ }_{42}^{2}$ Myongji University, Yongin, Kyonggido 449-728, Korea

${ }^{43}$ Nagasaki Institute of Applied Science, Nagasaki-shi, Nagasaki 851-0193, Japan

${ }_{44}$ University of New Mexico, Albuquerque, New Mexico 87131, USA

${ }^{45}$ New Mexico State University, Las Cruces, New Mexico 88003, USA

${ }^{46}$ Oak Ridge National Laboratory, Oak Ridge, Tennessee 37831, USA

${ }^{47}$ IPN-Orsay, Universite Paris Sud, CNRS-IN2P3, BP1, F-91406, Orsay, France ${ }^{48}$ Peking University, Beijing 100871, P. R. China

${ }^{49}$ PNPI, Petersburg Nuclear Physics Institute, Gatchina, Leningrad region, 188300, Russia

${ }^{50}$ RIKEN Nishina Center for Accelerator-Based Science, Wako, Saitama 351-0198, Japan

${ }^{51}$ RIKEN BNL Research Center, Brookhaven National Laboratory, Upton, New York 11973-5000, USA

${ }^{52}$ Physics Department, Rikkyo University, 3-34-1 Nishi-Ikebukuro, Toshima, Tokyo 171-8501, Japan

${ }^{53}$ Saint Petersburg State Polytechnic University, St. Petersburg, 195251 Russia

${ }^{54}$ Universidade de São Paulo, Instituto de Fúsica, Caixa Postal 66318, São Paulo CEP05315-970, Brazil

${ }^{55}$ Seoul National University, Seoul, Korea

${ }^{56}$ Chemistry Department, Stony Brook University, SUNY, Stony Brook, New York 11794-3400, USA

${ }^{57}$ Department of Physics and Astronomy, Stony Brook University, SUNY, Stony Brook, New York 11794-3400, USA

${ }^{58}$ University of Tennessee, Knoxville, Tennessee 37996, USA

${ }^{59}$ Department of Physics, Tokyo Institute of Technology, Oh-okayama, Meguro, Tokyo 152-8551, Japan

${ }^{60}$ Institute of Physics, University of Tsukuba, Tsukuba, Ibaraki 305, Japan

${ }^{61}$ Vanderbilt University, Nashville, Tennessee 37235, USA 


\author{
${ }^{62}$ Waseda University, Advanced Research Institute for Science and \\ Engineering, 17 Kikui-cho, Shinjuku-ku, Tokyo 162-0044, Japan \\ ${ }^{63}$ Weizmann Institute, Rehovot 76100, Israel \\ ${ }^{64}$ Institute for Particle and Nuclear Physics, Wigner Research Centre for Physics, Hungarian \\ Academy of Sciences (Wigner RCP, RMKI) H-1525 Budapest 114, POBox 49, Budapest, Hungary \\ ${ }^{65}$ Yonsei University, IPAP, Seoul 120-749, Korea
}

(Dated: March 14, 2012)

\begin{abstract}
Measurements of the anisotropy parameter $v_{2}$ of identified hadrons (pions, kaons, and protons) as a function of centrality, transverse momentum $p_{T}$, and transverse kinetic energy $\mathrm{KE}_{T}$ at midrapidity $(|\eta|<0.35)$ in $\mathrm{Au}+\mathrm{Au}$ collisions at $\sqrt{s_{N N}}=200 \mathrm{GeV}$ are presented. Pions and protons are identified up to $p_{T}=6 \mathrm{GeV} / c$, and kaons up to $p_{T}=4 \mathrm{GeV} / c$, by combining information from time-of-flight and aerogel Cerenkov detectors in the PHENIX Experiment. The scaling of $v_{2}$ with the number of valence quarks $\left(n_{q}\right)$ has been studied in different centrality bins as a function of transverse momentum and transverse kinetic energy. A deviation from previously observed quark-number scaling is observed at large values of $\mathrm{KE}_{T} / n_{q}$ in noncentral $\mathrm{Au}+\mathrm{Au}$ collisions $(20-60 \%)$, but this scaling remains valid in central collisions $(0-10 \%)$.
\end{abstract}

PACS numbers: 25.75.Dw, 25.75.Ld

\section{INTRODUCTION}

Measurements of the anisotropy parameter $v_{2}$ (the second coefficient in the Fourier expansion of the hadron yields with respect to the reaction plane) have played a pivotal role in the discovery of the strongly coupled quark-gluon plasma (sQGP) at RHIC [1-4]. At low $p_{T}$ $(\leq 2 \mathrm{GeV} / c)$ the agreement between ideal hydrodynamics calculations and the data have led to the conclusion that a near-perfect fluid is created in heavy-ion collisions at RHIC [5, 6]. Recent theoretical efforts aiming to quantify the ratio of the shear viscosity to the entropy density $\eta / s$ (see for example reviews in 7-9]) have confirmed that in the sQGP fluid this ratio is close to a conjectured quantum limit [10]. The high $p_{T}(\geq 6 \mathrm{GeV} / c)$ azimuthal anisotropies [11 13] have been attributed to the path-length dependence of energy loss in the medium and are used to constrain the theoretical descriptions of jet energy loss [14, 15]. At intermediate $p_{T}(2-6 \mathrm{GeV} / c)$, which is the focus of this paper, the identified hadron anisotropies have shown strong evidence for quark-like degrees of freedom and significant collectivity at the parton level. This is supported by the observation of scaling with the number of valence quarks in the hadron $\left(n_{q}\right.$ scaling) 16 21.

The scaling with number of valence quarks $\left(n_{q}\right)$ was seen as a confirmation of quark recombination as a novel particle-production mechanism that competes with fragmentation in the intermediate- $p_{T}$ range. Recombination models $22-26]$ were developed to account for the unusually large baryon-to-meson ratios (relative to $p+p$ collisions) and nuclear-modification factors [17, 27, 28], as well as the large elliptic flow at intermediate $p_{T}$, with pronounced differences between baryons and mesons.

\footnotetext{
${ }^{*}$ Deceased

${ }^{\dagger}$ PHENIX Spokesperson: jacak@skipper.physics.sunysb.edu
}

In the models, the $n_{q}$ scaling, which is manifested as $v_{2}^{\text {hadron }}\left(p_{T}\right) \approx n_{q} v_{2}\left(p_{T} / n_{q}\right)$, is an approximate scaling that comes from the addition of the valence-quark momenta at hadronization, with the assumption that the collective flow develops at the partonic level.

There are several theoretical considerations that suggest that the $n_{q}$ scaling should be violated in certain conditions. For example, the inclusion of higher Fock states describing the contribution of sea quarks and gluons have been shown to affect the $n_{q}$ scaling 29]. Similarly, models that consider recombination between "thermal" partons (soft partons thermalized in the medium) and "shower" partons (partons fragmented from jets) predict centrality-dependent deviations from $n_{q}$ scaling that are particle-species dependent [30]. Understanding the limits of the recombination domain is important in relation to viscous hydrodynamics and the extraction of the shear viscosity over entropy density $(\eta / s)$ from the data [31 33], as well as for developing a unified approach in describing jet energy loss and high $p_{T} v_{2}$ [34 36]. Searches for deviations from $n_{q}$ scaling are also important for the low-energy scan program at RHIC as they have been considered as a signature of the transition between sQGP formation and a hadronic system. Recent considerations of baryon transport may complicate this picture [37, which further reinforces the need for a detailed understanding of this scaling at $\sqrt{s_{N N}}=200 \mathrm{GeV}$.

The $n_{q}$ scaling has been tested in certain centralities and $p_{T}$ regions with identified particles [16 21]. However, the precision of experimental data on identified hadron $v_{2}$ is in many cases limited in statistics and $p_{T}$ reach, especially for baryon measurements at $\mathrm{KE}_{T} / n_{q}>1 \mathrm{GeV}$ $\left(\mathrm{KE}_{T}=\sqrt{p_{T}^{2}+m_{0}^{2}}-m_{0}\right)$ where the $n_{q}$ scaling may start to break. Therefore, the detailed $p_{T}$ limits and centrality dependence of the $n_{q}$ scaling have not been tested.

This paper reports on high-statistics measurements of the second order Fourier coefficient $v_{2}$ for identified pions $\left(\pi^{+}+\pi^{-}\right)$, kaons $\left(K^{+}+K^{-}\right)$, and protons $(p+\bar{p})$, which extend to relatively high $p_{T}$ (up to $6 \mathrm{GeV} / c$ for 
pions and protons and $4 \mathrm{GeV} / c$ for kaons). The data for different centrality events $(0-10 \%, 10-20 \%, 20-40 \%, 40-$ $60 \%$, and combinations thereof) are analyzed separately and the $n_{q}$ scaling is examined as a function of centrality. Comparisons with published measurements of $K_{S}^{0}$ and $\Lambda$ from STAR collaboration [21] are shown in the centralities $0-10 \%$ and $10-40 \%$. The experimental details are presented in Section [II the analysis methods are in Section III, the results and discussion are in Section IV. and Section $\nabla$ summarizes our findings.

\section{EXPERIMENTAL SETUP}

The PHENIX experiment is designed for the study of nuclear matter in extreme conditions through a variety of experimental observables. It comprises a tracking system optimized for the high-multiplicity environment of ultrarelativistic heavy ion collisions, a set of particle identification (PID) detectors, and a set of detectors aimed at determining the global properties of the collisions.

Figure 1 shows a schematic diagram of the PHENIX detector. The upper part is a beam-axis view of the two central spectrometer arms (West and East), covering the pseudorapidity region of $|\eta|<0.35$. Below that is a side view showing the two forward-rapidity muon arms (South and North) and the global detectors. A detailed description of the complete set of detectors can be found elsewhere [38].

The physics analysis presented here employed the tracking system [drift chamber (DC) and three layers of multiwire proportional chambers (PC1, PC2, and PC3)], the West arm time-of-flight detector ( $\mathrm{TOF} w)$, the aerogel Čerenkov counter (ACC), the ring imaging Cerenkov counter (RICH), the beam-beam counters (BBC), the reaction-plane detector (RxNP), and the muon piston calorimeter (MPC). Below, we give a brief description of each of these detector sub-systems and their role in the present analysis.

\section{A. Global Detectors}

The $\mathrm{BBC}$ are located at $\pm 144 \mathrm{~cm}$ from the nominal interaction point along the beam line in the pseudorapidity region $3.0<|\eta|<3.9$. Each BBC comprises 64 Cerenkov telescopes, arranged radially around the beam line. The BBCs provide the main collision trigger for the experiment and are used in the determination of the collision vertex position along the beam axis ( $z$-vertex) and the centrality of the collisions. They also provide the start time for the time-of-flight measurement with timing resolution of $\sigma_{\mathrm{BBC}}=37 \mathrm{ps}$.

The RxNP [39] was installed in PHENIX before the 2007 data-taking period. It is located at $\pm 38 \mathrm{~cm}$ from the nominal interaction point and has full azimuthal coverage. Each RxNP comprises two rings of plastic scintillator paddles, with each paddle subtending $\Delta \phi=\pi / 6$.

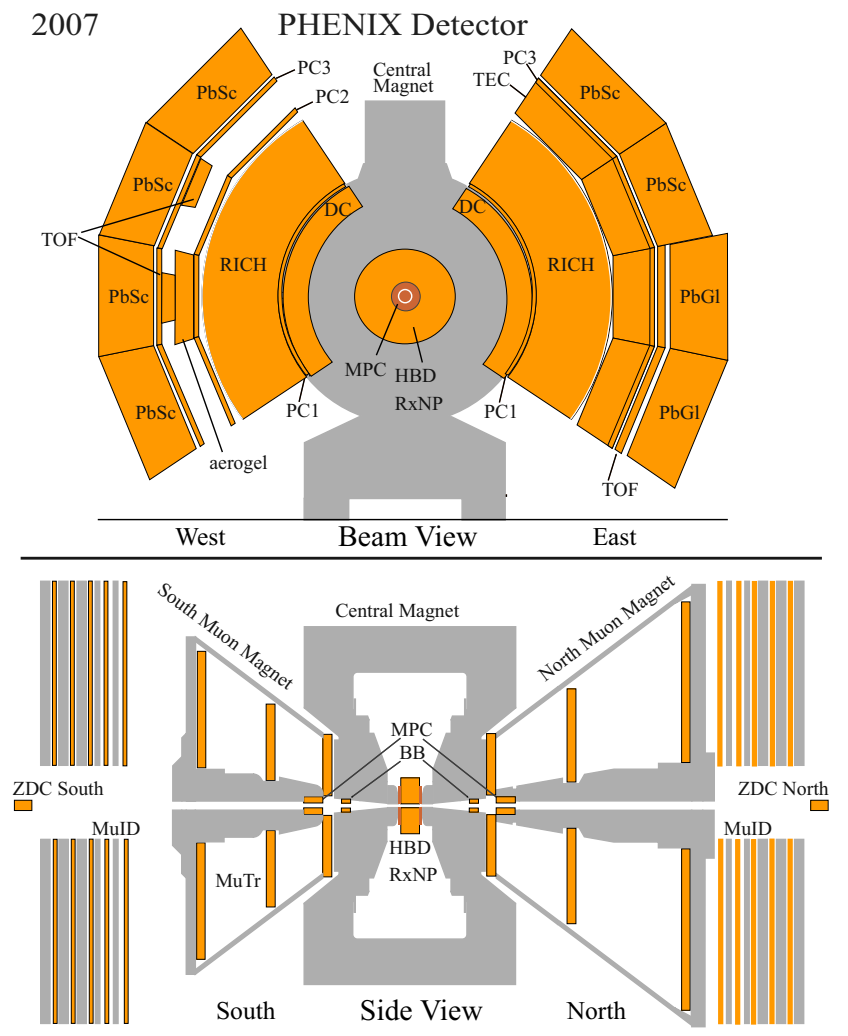

FIG. 1: (color online) The PHENIX detector configuration for RHIC 2007 data-taking period. The beam-beam counters $(\mathrm{BBC})$ are labeled as BB.

The inner and outer segments cover the pseudorapidity ranges $1.0<|\eta|<1.5$ and $1.5<|\eta|<2.8$, respectively. The RxNP is the main detector used in the event-plane determination for this analysis. The event-plane resolution $(\operatorname{Res}(\Psi))$ [40], which is used as a correction to the $v_{2}$ measurement, is defined as

$$
\operatorname{Res}(\Psi)=\left\langle\cos \left(2\left(\Psi-\Psi_{R P}\right)\right\rangle .\right.
$$

Here the bracket \langle\rangle indicates an average over all events, $\Psi_{R P}$ is true reaction plane (which is defined by the directions of interaction parameter and beam), and $\Psi$ is the event plane (which is measured by the detector event by event). A larger value of $\operatorname{Res}(\Psi)$ corresponds to a better measurement of the event-plane. In a given event, the event-plane resolution depends on the charged-particle multiplicity and the size of the azimuthal anisotropy signal; thus the resolution is centrality dependent. A resolution of up to $73 \%$ is achieved for midcentral events.

The MPC are electromagnetic calorimeters situated at $\pm 223 \mathrm{~cm}$ from the nominal interaction point inside the cylindrical openings at the front of the muon magnet pistons [41] and have $2 \pi$ azimuthal acceptance. The pseudorapidity coverage is about $3.0<\eta<3.8$ for the north side and $-3.7<\eta<-3.1$ for the south side. The MPCs are comprised of 220 modules in the north piston hole and 192 in the south with $\mathrm{PbWO}_{4}$ crystals and Avalanche Photodiode readouts, and can detect both charged and 
neutral particles. In this analysis, the MPCs were used for event-plane determination. Although the event-plane resolution (up to $50 \%$ in midcentral collisions) is lower than that achieved with the RxNP, the MPCs provide an important systematic check on the RxNP measurement due to their larger pseudorapidity separation from the central spectrometer and therefore smaller nonflow effects on the $v_{2}$ measurement.

\section{B. Tracking and Particle Identification Detectors}

The charged particle momentum is reconstructed in the tracking system comprised of the DC located outside of an axially-symmetric magnetic field at a radial distance between $2.0 \mathrm{~m}$ and $2.4 \mathrm{~m}$ followed by the PC1 with pixel-pad readout. The pattern recognition in the DC is based on a combinatorial Hough transform in the track bend plane. A track model based on a field-integral lookup table determines the charged particle momentum, the path length to the TOFw and a projection of the track to the outer detectors. The momentum resolution in this data set was estimated to be $\delta p / p \approx 1.3 \% \oplus 1.2 \% \times p$ $(\mathrm{GeV} / c)$, where the first term represents multiple scattering up to the DC and the second term is due to the DC spatial resolution. The momentum resolution is worse than that of previous data set is due to the weaker magnet configuration.

The tracks are matched to hits registered in the second and third layers of the pad chambers, PC2 and PC3, which are located at radial distances of $4.19 \mathrm{~m}$ and $4.98 \mathrm{~m}$ from the interaction point. Thus, the contribution of tracks originating from decays and $\gamma$-conversions is reduced.

To improve the track purity further, we employ the RICH, which is a threshold gas Cerenkov detector located in the radial region $2.5 \mathrm{~m}<r<4.1 \mathrm{~m}$. The Cerenkov radiator gas $\left(\mathrm{CO}_{2}\right)$ at atmospheric pressure has an index of refraction $n=1.000410\left(\gamma_{t h}=35\right)$, which corresponds to a momentum threshold of $20 \mathrm{MeV} / c$ for an electron and $4.65 \mathrm{GeV} / c$ for a pion. The RICH provides a veto for the electrons and positrons, which are predominantly pairs resulting from $\gamma$-conversions and Dalitz decays.

The primary PID used in this analysis is the TOFw, which is located at a radial distance of $4.81 \mathrm{~m}$ from the interaction point and covers the pseudorapidity range $|\eta|<0.35$ and $\delta \phi=22^{\circ}$ in azimuth. The TOFw was built using multigap resistive plate chamber technology (MRPC) [42] and installed in PHENIX before the 2007 data taking period. The MRPCs have six gas gaps formed by layered glass plates with thickness of $550 \mu \mathrm{m}$, separated by $230 \mu$ m-thick monofilament fishing line. The MRPCs are positioned in a gas volume and operated with a gas mixture of $95 \% \mathrm{R} 134 \mathrm{a}$ and $5 \%$ isobutane $\left(\mathrm{C}_{4} \mathrm{H}_{10}\right)$, and bias voltage of $14 \mathrm{kV}$. The TOFw system is composed of 128 MRPC modules each of which has four signal strips of size $37 \times 2.8 \mathrm{~cm}^{2}$ and separation of $0.3 \mathrm{~cm}$. The readout [43] is double-sided, which allows for hit positioning along the direction of the strip to be determined using the timing difference between the signals with resolution of the order $1 \mathrm{~cm}$. The other two hit coordinates are determined using the global position of the strips within PHENIX. The average time measured on both sides of the strips provides the stop time for the time-of-flight measurement. The timing resolution of the BBC-TOFw system was determined by selecting charged tracks (see Section IIIA) with momentum in the range $1.1 \mathrm{GeV} / c<p<1.5 \mathrm{GeV} / c$ and examining the timing difference between the measured flight-time and the time which is expected under the assumption that the particles are pions. The resulting time distribution is shown in Fig. 2. Since the pions dominate the total yield in this momentum region, a narrow peak centered around $t-t_{\text {expected }} \approx 0$ is observed. The other two broad peaks in Fig. 2 correspond to kaons and protons. A Gaussian distribution is fit to the pion peak and yields a resolution of $\sigma_{B B C-T O F w}=84 \pm 1$ ps for the BBC-TOFw system.

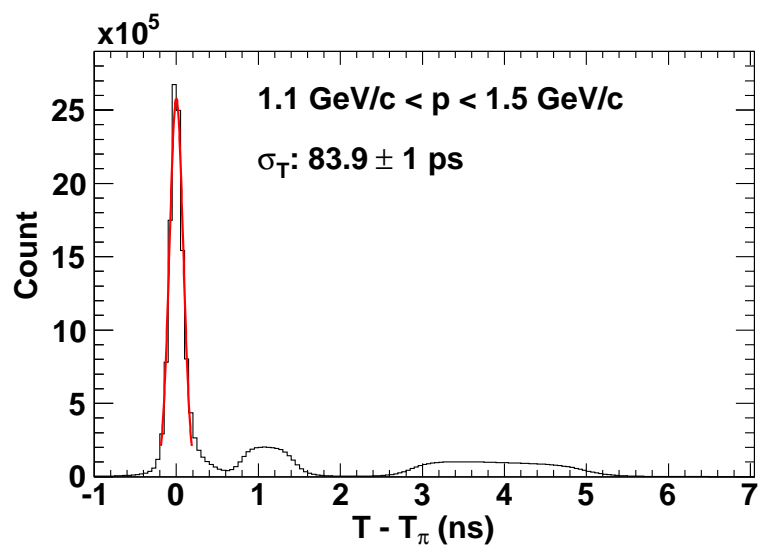

FIG. 2: (color online) Timing difference $T-T_{\pi}$, the difference between the measured time in the TOFw and the time calculated assuming each candidate track is a pion.

The excellent timing resolution allows for $4 \sigma$ separation in mass-squared reaching up to $p_{T}=2.5 \mathrm{GeV} / c$ for $\pi / K$, and up to $p_{T}=4 \mathrm{GeV} / c$ for $K / p$. The PID is further extended in $p_{T}$ by use of asymmetric cuts around the centroids of the mass-squared distributions.

TABLE I: The main characteristic parameters of TOFw and $\mathrm{ACC}$

\begin{tabular}{ccc}
\hline \hline & TOFw & ACC \\
\hline$\Delta \eta$ & $(-0.35,0.35)$ & $(-0.35,0.35)$ \\
$\Delta \phi(\mathrm{rad})$ & $(-0.061,0.110)$ & $(-0.108,0.156)$ \\
& $(0.503,0.674)$ & \\
Radial distance $(\mathrm{cm})$ & 481.36 & 449.4 \\
number of cells & 512 & 160 \\
cell size $\left(\mathrm{cm}^{2}\right)$ & $37 \times 2.8$ & $11.95 \times 23.10$ \\
\hline \hline
\end{tabular}

The ACC is used in conjunction with the TOFw to 
aid the PID at high $p_{T}$. It is situated in the West spectrometer arm in front of the TOFw detector. The ACC is a Čerenkov radiation detector with a relatively high index of refraction $\left(n=1.0113, \gamma_{t h}=8.5\right)$, which means that light is produced at relatively low momenta. The threshold for radiation is $1.0 \mathrm{GeV} / c$ for pions, $3.0 \mathrm{GeV} / c$ for kaons, and $6.0 \mathrm{GeV} / c$ for protons. The combined ACC-TOFw information allows for $\pi / K$ separation up to $p_{T}=4 \mathrm{GeV} / c$, and $K / p$ separation up to $p_{T}=6 \mathrm{GeV} / c$. The main characteristic parameters of TOFw and ACC can be found in the Table I

\section{ANALYSIS METHOD}

\section{A. Event and Track Selection}

The results reported here are obtained from an analysis of $4.8 \times 10^{9}$ minimum bias events obtained during the 2007 running period. The minimum-bias trigger is defined by a coincidence between North and South BBC signals and an energy threshold of one neutron in both the North and South zero-degree calorimeters [38]. The collision vertex $z$ is constrained to $|z|<30 \mathrm{~cm}$ of the origin of the coordinate system.

Charged tracks are selected based on the track quality information from the tracking system (DC-PC1). The tracks are then projected to the outer detectors and confirmed by requiring that the closest hit to the track projection is within certain spatial windows in $\phi$ and $z$. The distributions for the distance between the closest hit and projection in the azimuthal and $z$ directions are fitted with a double Gaussian function, one Gaussian function is for the signal distribution and the other for the background. For $p_{T}<3 \mathrm{GeV} / c$, hits are required to match the TOFw and the PC3 to within $2 \sigma$ from the signal's Gaussian distribution in $\phi$ and $z$. For $p_{T} \geq 3 \mathrm{GeV} / c$, hits are required to match the $\mathrm{PC} 2$ and the PC3 to within $3 \sigma$ and the TOFw to within $2 \sigma$ in $\phi$ and $z$. Background from $\gamma$-conversions is further reduced by applying a RICH veto. For the pions, this veto only works for $p_{T}<5 \mathrm{GeV} / c$ since pions with $p_{T}$ higher than that will fire the RICH. To evaluate the residual background, remaining after these selections, the backgroundto-signal (B/S) ratios from the double Gaussian function fitting within the samples selected for the analysis are examined. For $p_{T}<3 \mathrm{GeV} / c$ the background comprises less than $1 \%$ of the selected tracks. At higher $p_{T}$ the background increases, reaching $\mathrm{B} / \mathrm{S} \approx 7 \%$ for $5.5 \mathrm{GeV} / c<p_{T}<6.0 \mathrm{GeV} / c$ in the $0-20 \%$ centrality bin.

\section{B. Particle Identification}

The particles are identified by their mass, based on measurements of the momentum, the time-of-flight to the TOFw detector, and the path-length along the trajectory. PID selections are performed by applying momentum-dependent cuts in mass-squared. The masssquared distributions are fit with a 3-Gaussian function corresponding to pions, kaons, and protons. The corresponding widths and centroids are extracted from the data as a function of transverse momentum. In the calibration process, we ensure that the centroids of these distributions do not move as a function of $p_{T}$ and that the widths vary as expected from the known momentum and timing resolution of the detector. We then select a sample from each particle species aiming for at least $90 \%$ purity in PID. The high purity of the sample will allow us to measure the $v_{2}$ of selected particles accurately and minimize the uncertainty resulting from PID contamination. At lower transverse momenta $\left(p_{T}<2.5 \mathrm{GeV} / c\right)$, the $2 \sigma$ bands centered around each particle's $m^{2}$ do not overlap, thus symmetric cuts, $m_{0}^{2}-2 \sigma<m^{2}<m_{0}^{2}+2 \sigma$, allow for PID with high purity. In the range $2.5 \mathrm{GeV} / c<p_{T}<3 \mathrm{GeV} / c$, the $\pi / K$ separation is achieved by excluding the particles that lie within $2 \sigma$ of the centroid of the mass-squared distribution of another particle. This procedure is demonstrated in Fig. 3 where the PID selections for $\pi, K$, and $p$ are shown with the hatched areas in the plot. The Gaussian fits to the individual $\mathrm{m}^{2}$ peaks (dashed-line curves) and the combined fit to the entire $m^{2}$ distribution (solid line) are also shown.

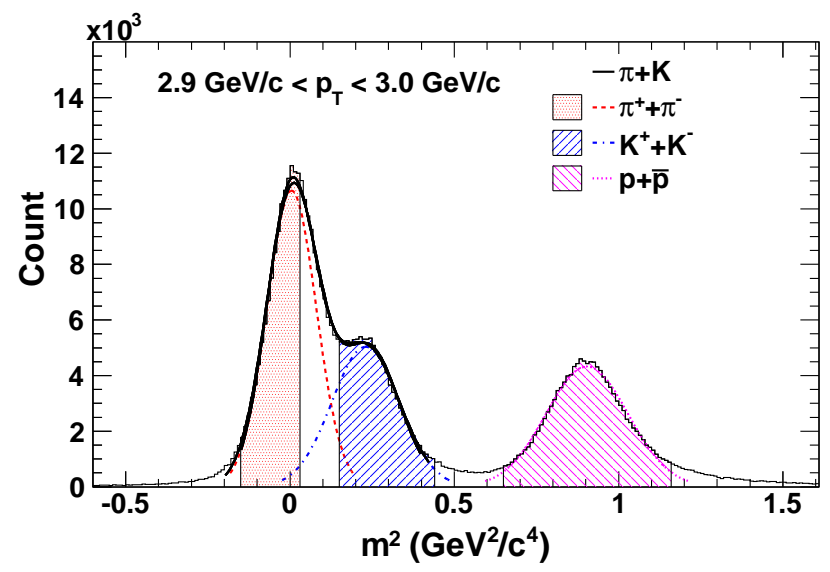

FIG. 3: (color online) The mass-squared distribution measured by TOFw in the $p_{T}$ region $2.9 \mathrm{GeV} / c<$ $p_{T}<3.0 \mathrm{GeV} / c$. The hatched areas show the pion, kaon, and proton selections, from left to right. The dashed lines show Gaussian fits to the individual $\mathrm{m}^{2}$ peaks, while the solid line represents a combined fit to the $m^{2}$ distribution including the pions and kaons.

At higher transverse momentum $3 \mathrm{GeV} / c<$ $p_{T}<6 \mathrm{GeV} / c$, the lower $m^{2}$ range of the pion distribution remains relatively unaffected by contamination from kaons and protons. Therefore, a pion sample with purity better than $90 \%$ can be selected based on information from the TOFw alone, by applying the $m^{2}$ cuts indicated in panels (a) and (c) of Fig. [4, and listed in Table II 


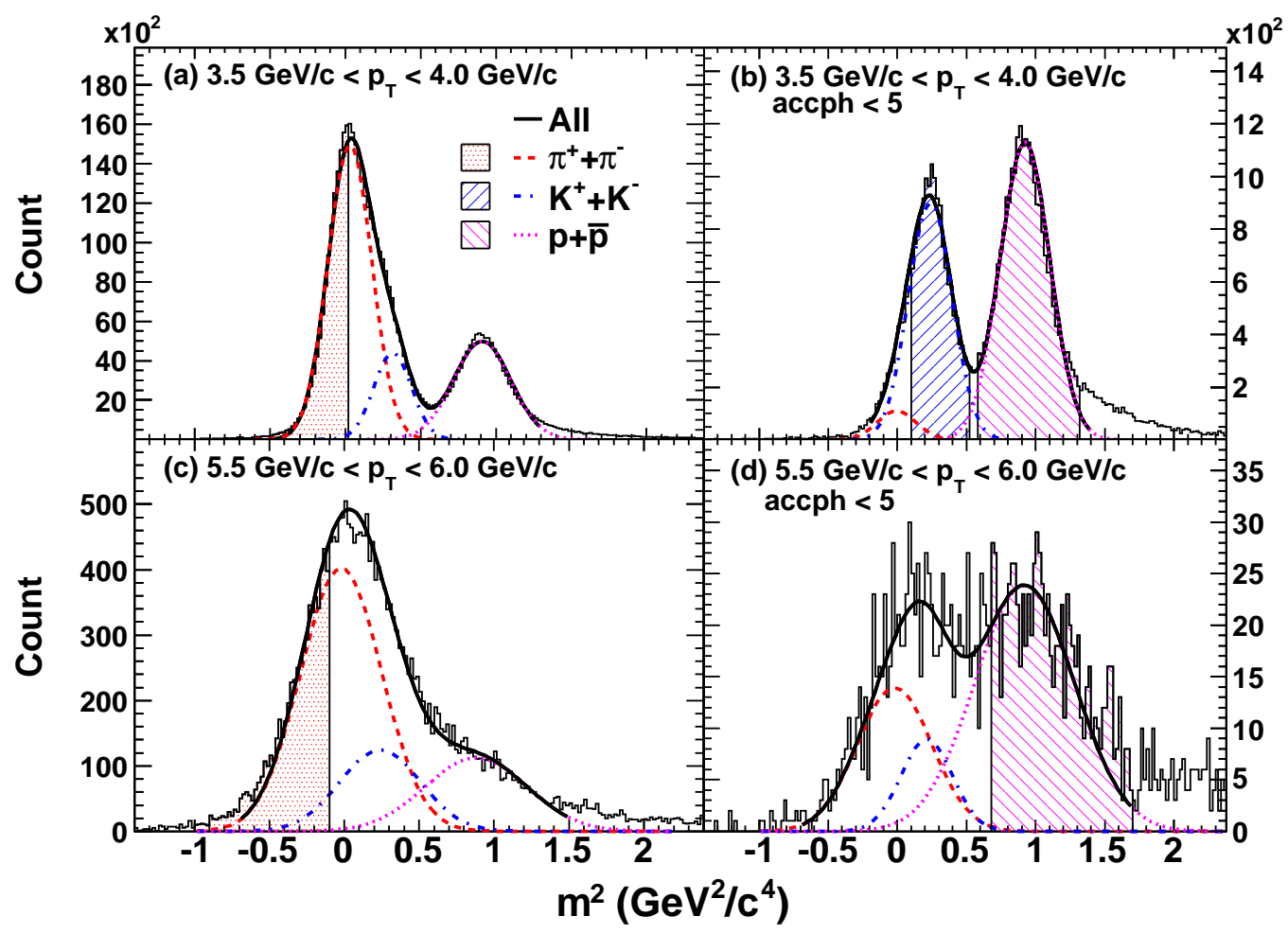

FIG. 4: (color online) The mass-squared distribution in the TOFw without (left panels) and with (right panels) the ACC photon yield (accph) cuts for different $p_{T}$ regions. The hatched areas show the $m^{2}$ cuts used for pion, kaon, and proton selections. The distribution is fit with a 3 -Gaussian function (solid line). The individual Gaussian distributions corresponding to $\pi, K$, and $p$ and are as dashed lines.

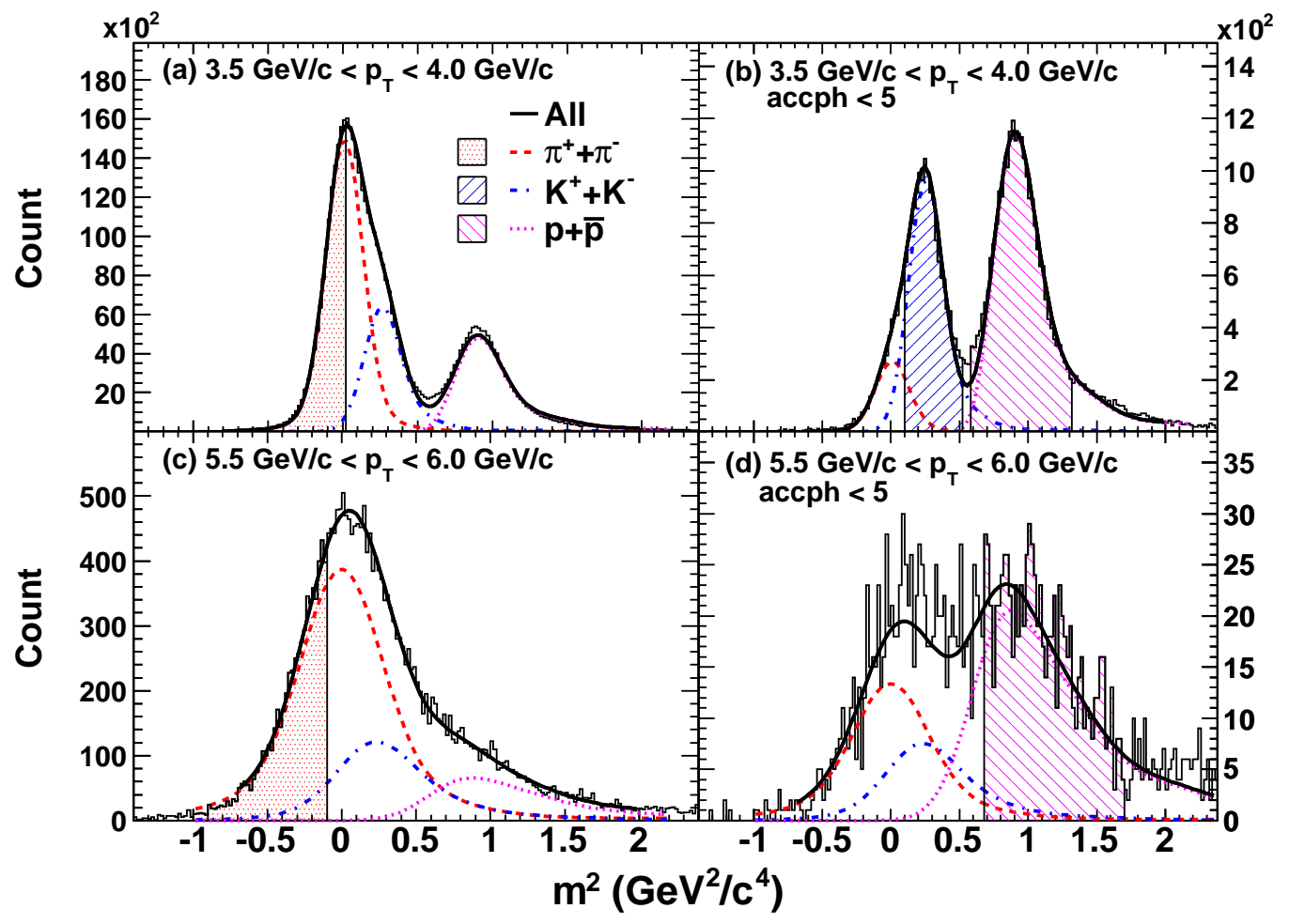

FIG. 5: (color online) The mass-squared distribution in the TOFw without (left panels) and with (right panels) the ACC photon yield (accph) cuts for different $p_{T}$ regions. The distribution is fit with the sum of three empirical $m^{2}$ distribution functions that are propagated from sampling a Landau shape momentum distribution as described in the text (solid line). The individual distributions corresponding to $\pi, K$, and $p$ are shown with dashed lines. 
For kaon and proton identification at $p_{T}>3 \mathrm{GeV} / c$, the ACC is used in conjunction with the TOFw detector, as shown in Fig. 4 panels (b) and (d). The turnon momenta of the ACC for pions, kaons, and protons are $1.0 \mathrm{GeV} / c, 3.0 \mathrm{GeV} / c$, and $6.0 \mathrm{GeV} / c$, respectively. This turn-on is gradual, with the number of photons registered per photomultiplier tube (PMT) growing up to 15 for pions, and 10 (kaons and protons) as the hadrons exceed their respective threshold momentum by $\approx 1 \mathrm{GeV} / c$. With this information, the photon yield from the ACC can be used as a rejection veto based on whether it is "on" $($ accph $\geq 5)$ or "off" $($ accph $<5)$. Due to the occupancy effects in the ACC as well as the spatial resolution of track projection to the ACC, the pions cannot be rejected completely. The effect of this veto cut is demonstrated in Fig. 4. panels (b) and (d). The pion rejection by the ACC in combination with asymmetric $m^{2}$ cuts, which are indicated here and listed in Table [I] allow for kaon and proton PID up to $p_{T}$ of 4 and $6 \mathrm{GeV} / c$, respectively.

We use the Gaussian fits to the mass-squared distributions to estimate the PID purity in the selected $m^{2}$ regions. This is straightforward at $p_{T}<3 \mathrm{GeV} / c$, where the peaks associated with each particle are well defined. At higher $p_{T}$ the uncertainties are larger, since the pion and kaon peaks merge and the individual yields are not well constrained. We have checked that for $p_{T}>4 \mathrm{GeV} / c$, after efficiency corrections, the $K / \pi$ ratio obtained from our fits is consistent with the measurements of the $K / \pi\left(K_{S}^{0} / \pi\right)$ ratio by the STAR experiment within the statistic and systematic uncertainties. At $p_{T}=5.22 \mathrm{GeV} / c$, the $K / \pi$ ratio is reported as $0.326 \pm 0.013$ (stat) \pm 0.134 (syst) and the $K_{S}^{0} / \pi$ ratio is reported as $0.435 \pm 0.022$ (stat) \pm 0.072 (syst) in $p+p$ collisions at $\sqrt{s_{N N}}=200 \mathrm{GeV}$; the ratios in $p+p$ and $\mathrm{Au}+\mathrm{Au}$ collisions are similar [44 46]. In our study, the kaon contamination in the pion sample is relatively insensitive to the kaon yield. For example, if we artificially increase the kaon yield by $30 \%$, the contamination in the pion sample increases from $7 \%$ to $9 \%$.

The $m^{2}$ distributions are not strictly Gaussian shape, but have tails extending to the higher mass region. This effect is not noticeable at low $p_{T}$ but comes into prominence at intermediate and high $p_{T}$. Hadrons coming from resonance decays may survive the tracking cuts but will have misreconstructed momentum and contribute to this high mass tail. The total momentum distribution of hadrons, including those from resonance decays, is much closer to a Landau distribution than a Gaussian distribution. To get an estimate of the possible PID contamination in this case, we have fit the $m^{2}$ distribution with an empirical function that was determined by sampling a momentum distribution with a Landau shape instead of a Gaussian. This empirical $\mathrm{m}^{2}$ distribution is found to give a much better fit than a simple 3-Gaussian function and it gives a good description of the high mass tails. Finally, we reevaluate the PID contamination with this empirical function. An example of these fits is shown in
Fig. 5. The tail of $m^{2}$ distribution is well described by the empirical pion, kaon, and proton $m^{2}$ functions which are presented with different dashed lines. In this case, at high $p_{T}$ the contamination from kaons and pions in the proton sample increases to $9 \%$ from $1 \%$ in the case of the Gaussian fits.

The PID purity for each particle species estimated in different $p_{T}$ ranges is listed in Table [I. These estimates reflect the values obtained for the $0-20 \%$ central $\mathrm{Au}+\mathrm{Au}$ collisions and are meant to provide lower limits for the measurements presented here. The purity in more peripheral collisions was found to be slightly better.

\section{Measurement of $v_{2}$}

The measurement of the anisotropy parameter $v_{2}$ aims to determine the event-by-event particle azimuthal correlation with the reaction plane of the collision. The true reaction plane, which is defined as the plane formed by the impact parameter $b$ and the beam direction, is not known experimentally. In addition, there exist other sources of correlations in azimuth, such as the correlations from resonance decays, jets, and quantum effects. These correlations, which are not related to the reaction plane, are called nonflow correlations. The goal is to determine the second coefficient in the Fourier expansion $v_{2}$ of the particle azimuthal distribution with respect to the reaction plane with minimal effects from nonflow correlations. To estimate the reaction plane angle $\Psi_{R P}$, we employ the event-plane method [40], in which the second harmonic azimuthal anisotropy signal determines the event-plane angle $\Psi$ based on hits registered in one of the event-plane detectors: RxNP or MPC. For an ideal detector, the measured distribution of event-plane angles should be isotropic. However, the actual measurement is usually affected by finite acceptance and nonuniform efficiencies. We apply a standard event-plane flattening technique [16, 19, 40, 47] to remove the residual nonuniformities in the distribution of event-plane angles. The accuracy with which the event-plane angle can be determined depends on the strength of the $v_{2}$ signal and the multiplicity of the events in each centrality class. It is maximal for midcentral events, where both of these quantities are relatively large. The $v_{2}\left(p_{T}\right)$ measurement is performed by correlating the particle azimuthal angle $\varphi$ with the second harmonic event-plane angle $\Psi$, and correcting the observed signal for the event-plane resolution as follows:

$$
v_{2}=\frac{\langle\cos (2(\varphi-\Psi))\rangle}{\operatorname{Res}(\Psi)}
$$

Here the brackets \langle\rangle indicate an average over all particles in all events.

Since the true reaction plane angle is not directly measurable, the resolution correction is estimated using subevent techniques [40]. There are several different options 
TABLE II: The particle identification cuts in TOFw and ACC with PID purity in Au+Au collisions for the centralities 0-20\% and $20-60 \%$.

\begin{tabular}{|c|c|c|c|c|c|}
\hline \multirow[t]{2}{*}{ Particle } & \multirow{2}{*}{$\begin{array}{c}p_{T} \text { range } \\
(\mathrm{GeV} / c)\end{array}$} & \multirow{2}{*}{$\begin{array}{l}\text { TOFw Cut } \\
\left(\mathrm{GeV} / c^{2}\right)^{2}\end{array}$} & \multirow[t]{2}{*}{$\mathrm{ACC}$ Cut } & \multicolumn{2}{|c|}{ Purity } \\
\hline & & & & $0-20 \%$ & $20-60 \%$ \\
\hline \multirow[t]{3}{*}{ pion } & $<3$ & $m_{\pi}^{2} \pm 2 \sigma_{m_{\pi}^{2}}$ & None & $99 \%$ & $99 \%$ \\
\hline & {$[3.0,5.0)$} & {$[-1.0,0.0]$} & None & $95 \%$ & $96 \%$ \\
\hline & {$[5.0,6.0)$} & {$[-1.0,-0.1]$} & None & $91 \%$ & $92 \%$ \\
\hline \multirow{2}{*}{ kaon } & {$[3.0,3.5)$} & {$[0.1,0.5]$} & accph $<5.0$ & $94 \%$ & $95 \%$ \\
\hline & {$[3.5,4.0)$} & {$[0.1,0.5]$} & accph $<5.0$ & $91 \%$ & $92 \%$ \\
\hline \multirow[t]{2}{*}{ proton } & $<3$ & $m_{p}^{2} \pm 2 \sigma_{m_{p}^{2}}$ & None & $99 \%$ & $99 \%$ \\
\hline & {$[3.0,4.0)$} & {$[0.6,1.3]$} & accph $<5.0$ & $97 \%$ & $98 \%$ \\
\hline
\end{tabular}

in using the sub-event techniques. The present analysis uses the two sub-event and the three sub-event methods. These methods are compared to evaluate the systematic uncertainties associated with the event-plane resolution corrections.

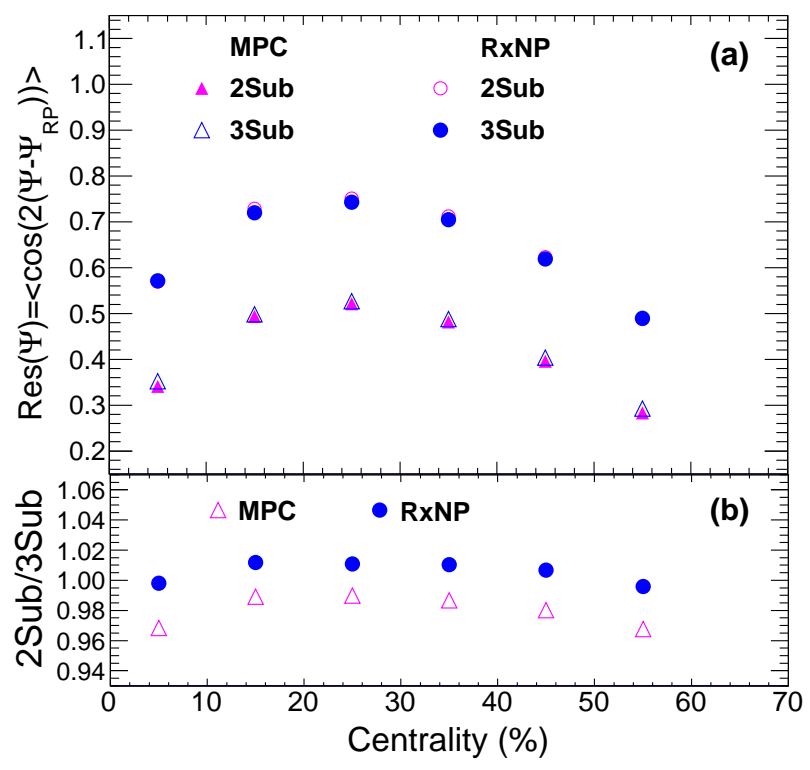

FIG. 6: (color online) Panel (a) shows the event-plane resolution as a function of centrality for the RxNP and the MPC detectors. Panel (b) shows the ratio of the event-plane resolution obtained from two sub-events and three sub-events as a function of centrality.

The RxNP and the MPC detectors each have two subdetectors, North and South, which are positioned symmetrically around the origin of the nominal collision point with equal acceptance in pseudorapidity. Thus, they pro- vide a natural two sub-event division. The correlation between the event-plane angles determined from the North and South sub-detectors, $\Psi_{N}$ and $\Psi_{S}$, allows for the estimate of the resolutions corrections as follows:

$$
\operatorname{Res}\left(\Psi_{N}\right)=\operatorname{Res}\left(\Psi_{S}\right)=\sqrt{\left\langle\cos 2\left(\Psi_{S}-\Psi_{N}\right)\right\rangle}
$$

The resolution correction can also be expressed analytically [40] as:

$$
\begin{gathered}
\operatorname{Res}(\Psi)=\left\langle\cos 2\left(\Psi-\Psi_{R P}\right)\right\rangle= \\
\frac{\sqrt{\pi}}{2} \chi e^{-\frac{\chi^{2}}{2}}\left[I_{0}\left(\frac{\chi^{2}}{2}\right)+I_{1}\left(\frac{\chi^{2}}{2}\right)\right]
\end{gathered}
$$

where $I_{0}$ and $I_{1}$ are modified Bessel functions. The parameter $\chi=v_{2} \sqrt{2 M}$, where $M$ is the number of particles used to determine the event-plane, describes the dispersion of the flow vector. With the use of Equation 3 and Equation 4, we obtain the sub-event parameters $\chi_{S}$ and $\chi_{N}$. Subsequently, to optimize the event-plane resolution, the two sub-events are combined, and the full event parameter is taken as $\chi=\sqrt{2} \chi_{S}=\sqrt{2} \chi_{N}$. This procedure relies on the two sub-events being equal in multiplicity and registering the same size $v_{2}$ signal, which may not be the case experimentally. To avoid this uncertainty, we also use a three sub-events technique to determine the event-plane resolution with Equation [5] [40]. To determine the event-plane resolution of RxNP detector (sub-event A), we employ information from the North and South portions of the MPC detector (sub-events B and $\mathrm{C}$ ). In turn, to estimate the resolution of the MPC detector, the North and South portions of the RxNP detector are used to provide sub-events B and C.

$$
\operatorname{Res}\left(\Psi_{A}\right)=\left\langle\cos 2\left(\Psi_{A}-\Psi_{R P}\right)\right\rangle=
$$




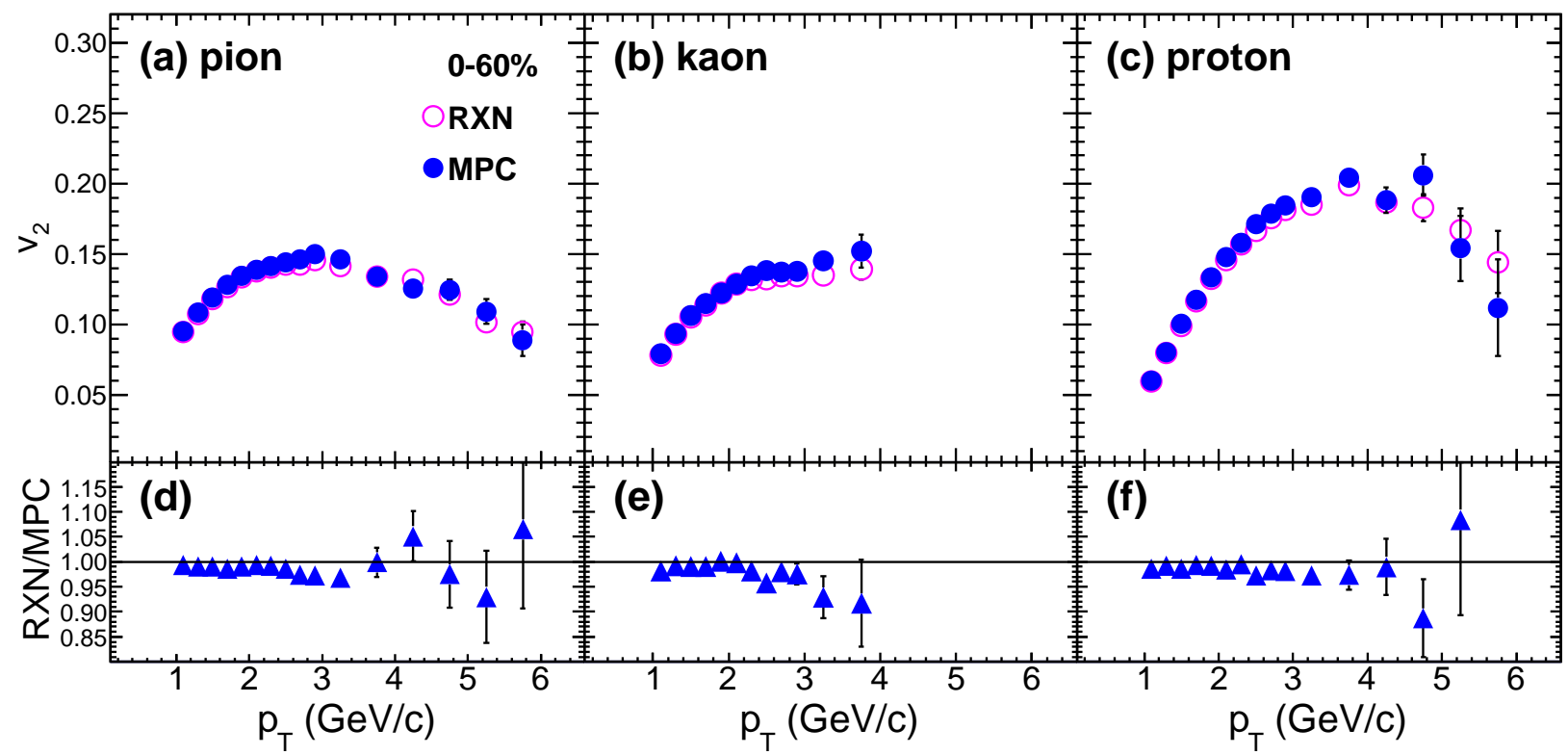

FIG. 7: (color online) The upper panels show the azimuthal anisotropy $v_{2}\left(p_{T}\right)$ of pions (a), kaons (b), and protons (c) in the $0-60 \%$ centrality class measured with respect to event-planes determined by the MPC (closed symbols) or the RxNP (open symbols) detectors. The event-plane resolution is estimated by the three sub-events method. The ratio of $v_{2}(\mathrm{RxNP})$ to $v_{2}$ (MPC) is shown in the lower panels as a function of $p_{T}$ for pions (d), kaons (e), and protons (f).

$$
\sqrt{\frac{\left\langle\cos 2\left(\Psi_{A}-\Psi_{B}\right)\right\rangle\left\langle\cos 2\left(\Psi_{A}-\Psi_{C}\right)\right\rangle}{\left\langle\cos 2\left(\Psi_{B}-\Psi_{C}\right)\right\rangle}}
$$

The event-plane resolution for the RxNP (circles) and the MPC (triangles) detectors obtained with the above procedures are shown as a function of the event centrality in Fig. 6(a). The results show the expected trend, with maximal resolution for the 20-30\% centrality class where both the event multiplicity and the $v_{2}$ signal are large, and a decrease for the more central events (due to lower $v_{2}$ strength), and more peripheral events (due to smaller multiplicity). Figure 6(b) shows the ratio of the results obtained with the two sub-event and the three sub-event techniques. The results for the RxNP detector (closed symbols) agree to within $2 \%$. A larger difference (up to $4 \%$ ) is observed for the MPC detector (open symbols) which is mainly due to the asymmetric pseudorapidity coverage of the MPC. The event-plane resolution from three sub-events method is used to correct the $v_{2}$ measurement.

From Fig. 6 it is evident that the RxNP detector has better resolution for the event-plane angle, as well as smaller systematic uncertainty in the event-plane determination than the MPC detector. Therefore, it is desirable to use the RxNP for the $v_{2}$ measurement. One possible disadvantage of the RxNP over the MPC detector is the smaller pseudorapidity separation from the central spectrometer $(|\eta|<0.35)$, which makes the $v_{2}$ measurement more susceptible to nonflow correlations caused by jets. Since the results presented here aim to study the high $p_{T}$ azimuthal anisotropies of identified charged hadrons and the limits of $n_{q}$ scaling, it is particularly important to minimize such effects. To evaluate the nonflow contributions we examine the $v_{2}\left(p_{T}\right)$ distributions for pions, kaons, and protons measured using the MPC and the RxNP detectors independently. For the $p_{T}<6 \mathrm{GeV} / c$, a previous study indicated that nonflow effects are small for the event-plane measured by the BBC detectors, which have a pseudorapidity coverage similar to that of the MPC detectors [48]. Figure 7 shows the results in the $0-60 \%$ centrality range for each particle species (upper panels), and the ratio of the results obtained with the two event-plane detectors (lower panels). Non-flow correlations are expected to enhance the measured $v_{2}$ signal for the detector which is more affected, especially in the higher $p_{T}$ range. We do not find any evidence for a significant increase in nonflow contributions in the measurement based on the RxNP detector.

Based on these considerations, the results presented in Section IV are based on the reaction plane measured solely by the RxNP, taking advantage of its better eventplane resolution in comparison to the MPC.

\section{Systematic Uncertainties in $v_{2}$}

The systematic uncertainties in the $v_{2}$ measurement obtained with the RxNP detector can be broadly characterized according to the following categories: 1) eventplane resolution corrections; 2) event-plane measured 
TABLE III: Systematic uncertainties given in percent on the $v_{2}$ measurements.

\begin{tabular}{|c|c|c|c|c|}
\hline Error Sources & Species & $0-20 \%$ & $20-60 \%$ & Туре \\
\hline Event-plane detectors & & $3 \%$ in $p_{T} 1-3 \mathrm{GeV} / c$ & $3 \%$ in $p_{T} 1-5 \mathrm{GeV} / c$ & $\mathrm{~B}$ \\
\hline \multirow[t]{3}{*}{ Background } & pion & $1 \%$ in $p_{T} 1-4 \mathrm{GeV} / c$ & $1 \%$ in $p_{T} 1-4 \mathrm{GeV} / c$ & $\mathrm{~A}$ \\
\hline & & $4 \%$ in $p_{T} 4-6 \mathrm{GeV} / c$ & $3 \%$ in $p_{T} 4-6 \mathrm{GeV} / c$ & \\
\hline & & $5 \%$ in $p_{T} 4-6 \mathrm{GeV} / c$ & $3 \%$ in $p_{T} 4-6 \mathrm{GeV} / c$ & \\
\hline \multirow[t]{2}{*}{ PID } & pion & \multicolumn{2}{|c|}{$\begin{array}{l}\text { negligible in } p_{T} 1-3 \mathrm{GeV} / c \\
2 \% \text { in } p_{T} 3-6 \mathrm{GeV} / c\end{array}$} & $\mathrm{~A}$ \\
\hline & kaon & \multicolumn{2}{|c|}{$\begin{array}{l}\text { negligible in } p_{T} 1-3 \mathrm{GeV} / c \\
\qquad 2 \% \text { in } p_{T} 3-4 \mathrm{GeV} / c\end{array}$} & A \\
\hline Acceptance & & $8 \%$ & $3 \%$ & $\mathrm{C}$ \\
\hline and run-by-run & & & & \\
\hline
\end{tabular}

from different detectors; 3) $v_{2}$ from background tracks; 4) PID purity; and 5) acceptance and run-by-run dependencies.

The uncertainties stemming from the event-plane resolution corrections are independent of particle species and $p_{T}$. They are found to be around $2 \%$ for all centralities by studying the event-plane resolution difference for the RxNP with the two and three sub-event methods.

The uncertainties from event-planes measured with different detectors (RxNP, MPC) are found to be independent of the particle species, by comparing the results from RxNP and MPC. In the 0-20\% centrality class, we assign a $3 \%$ systematic uncertainty for $p_{T}<3 \mathrm{GeV} / c$ and a $5 \%$ systematic uncertainty for $p_{T}>3 \mathrm{GeV} / c$. In the $20-60 \%$ centrality class, we assign a $3 \%$ systematic uncertainty for $p_{T}<5 \mathrm{GeV} / c$ and a $5 \%$ systematic uncertainty for $p_{T}>5 \mathrm{GeV} / c$.

Background tracks that are not removed by the tracking and PID selections outlined in Sections III A and III B may influence the measured $v_{2}$ if they carry a signal which is different from the particle of interest. The background tracks may come from decays, $\gamma$-conversions, or false track reconstruction. The backgrounds are centrality dependent, and may also have $p_{T}$ and hadron species dependence. A sample of background-dominated tracks was selected based on the normalized distance between the hits registered in the TOFw detector and the track projections. Specifically, a $4 \sigma-10 \sigma$ window in the $z$ direction was utilized. The azimuthal anisotropy of the background was then measured following the procedure used for the signal. For $p_{T} \approx 3 \mathrm{GeV} / c$, the $v_{2}$ of the background is similar to that of the pion, but it decreases at higher $p_{T}$ down to about $60 \%$ of the $v_{2}$ of the pion (or $30 \%$ of the $v_{2}$ of the proton) for $p_{T} \approx 6 \mathrm{GeV} / c$ in the 0
$20 \%$ centrality class. For pions, the resulting systematic uncertainties in $v_{2}$ are of the order $1 \%$ for $p_{T}<4 \mathrm{GeV} / c$ and reach up to $4 \%(3 \%)$ for $p_{T} \approx 6 \mathrm{GeV} / c$ for centrality 0-20\% (20-60\%). For protons, the resulting systematic uncertainties in $v_{2}$ are of the order $1 \%$ for $p_{T}<4 \mathrm{GeV} / c$ and reach up to $5 \%(3 \%)$ for $p_{T} \approx 6 \mathrm{GeV} / c$ for centrality 0-20\% (20-60\%).

The systematic uncertainties in $v_{2}$ resulting from hadron misidentification are based on the PID purity estimates listed in Table II and the size of $v_{2}$ of each species. For example, at $p_{T}=6.0 \mathrm{GeV} / c$ for $0-20 \%$ centrality, the protons purity is around $91 \%$ and the $v_{2}$ of pions and kaons are around $50 \%$ of that of the proton. We assign a $5 \%$ systematic uncertainty attributable to this effect. For $p_{T}<3 \mathrm{GeV} / c$, the uncertainties in $v_{2}$ due to PID contamination are negligible for all particle species. At higher $p_{T}$ the uncertainties in $v_{2}$ remain below $\approx 2 \%$ for kaons and pions; for protons with $p_{T}>4 \mathrm{GeV} / c$ these uncertainties reach up to $\approx 5 \%(3 \%)$ for centrality $0-20 \%$ (20-60\%).

Additional systematic checks were performed using different subsets of the detector, and data obtained with different magnetic field configurations. Differences of order $8 \%(3 \%)$ were found for the $0-20 \%$ (20-60\%) centrality, which have weak $p_{T}$ and particle species dependence.

Table III lists the summary of all these systematic uncertainties which are categorized by the types:

(A) point-to-point error uncorrelated between $p_{T}$ bins,

(B) $p_{T}$ correlated, all points move in the same direction but not by the same factor,

(C) an overall normalization error in which all points move by the same factor independent of $p_{T}$. 

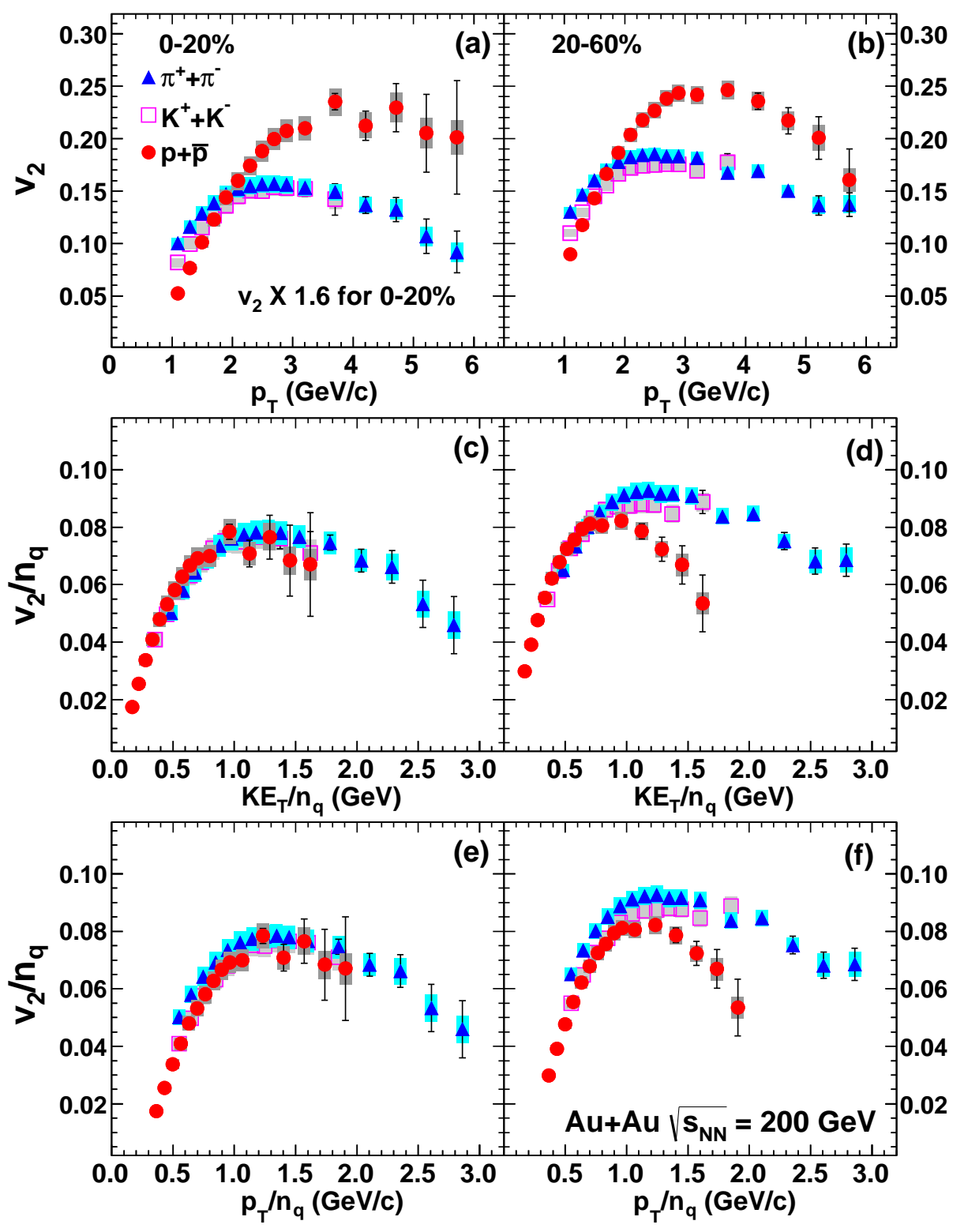

FIG. 8: (color online) Identified hadron $v_{2}$ in central (0-20\% centrality, left panels) and midcentral (20-60\%, right panels) $\mathrm{Au}+\mathrm{Au}$ collisions at $\sqrt{s_{N N}}=200 \mathrm{GeV}$. Panels (a) and (b) show $v_{2}$ as a function of transverse momentum $p_{T}$. Panels (c) and (d) show the quark-number-scaled $v_{2}\left(v_{2} / n_{q}\right)$ as a function of the kinetic energy per quark, $\mathrm{KE}_{T} / n_{q}$. Panels (e) and (f) show $v_{2} / n_{q}$ as a function of transverse momentum per quark, $p_{T} / n_{q}$. The $v_{2}$ of all species for centrality $0-20 \%$ has been scaled up by a factor of 1.6 for better comparison with results of $20-60 \%$ centrality. The error bars (shaded boxes) represent the statistical (systematic) uncertainties. The systematic uncertainties shown are type A and B only.

\section{RESULTS AND DISCUSSION}

The results for $v_{2}$ of identified pions, kaons, and protons are presented in Fig. 8 the results in central collisions (0-20\%) are presented in panels (a), (c), and (e) and the results in noncentral collisions $(20-60 \%)$ are presented in panels (b), (d), and (f). The symbols representing the different particle species are closed triangles for pions, open squares for kaons, and closed circles for protons. In order to better compare between two centralities, the $v_{2}$ of all species in the $0-20 \%$ centrality has been scaled up by a factor of 1.6. The error bars (shaded boxes) represent the statistical (systematic) uncertainties. The systematic uncertainties shown are type $\mathrm{A}$ and B only. Not shown are the type C systematic uncertainties, which are from the event-plane resolution, geometrical acceptance, and run-by-run dependence are around $8.5 \%(3.5 \%)$ for $0-20 \%(20-60 \%)$ centrality for all species 
at all values of $p_{T}$.

Panels (a) and (b) of Fig. 8 show $v_{2}\left(p_{T}\right)$. For both centrality selections, the $v_{2}$ values of pions and kaons are very similar in intermediate $p_{T}$ range $(2-4 \mathrm{GeV} / c)$, where the measured $v_{2}$ is maximal and is relatively independent of transverse momentum. Above $p_{T} \approx 4 \mathrm{GeV} / c$ the pion $v_{2}$ gradually decreases to a value which is comparable to the signal measured at $p_{T} \approx 1 \mathrm{GeV} / c$. In contrast, the proton $v_{2}\left(p_{T}\right)$ has a shape which is centrality dependent. In central collisions $(0-20 \%)$ the proton $v_{2}$ rises up to $p_{T} \approx 3.5 \mathrm{GeV} / c$ and then saturates at a value higher than the $v_{2}$ of pions. For noncentral collisions, the behavior is different: a decrease is observed in the proton $v_{2}$ for $p_{T}>4 \mathrm{GeV} / c$ leading to near equal $v_{2}$ signals for pions and protons at $p_{T} \approx 6 \mathrm{GeV} / c$.

The use of the $\mathrm{KE}_{T}$ variable was introduced in Reference [18], which is found to better represent the number of quark scaling behavior than $p_{T}$ at lower $p_{T}$. In panels (c) and (d) of Fig. 8 the $v_{2}$ signals have been scaled by the number of constituent quarks $n_{q}$ in the hadrons and are shown as a function of the transverse kinetic energy per quark $\mathrm{KE}_{T} / n_{q}$. A very different behavior is observed in central (Fig. 8(c)) and in noncentral (Fig. 8(d)) collisions. In the measured $p_{T}$ range, a universal behavior is seen in the central collisions within the statistical and systematic uncertainties, but not in the noncentral collisions, where the $v_{2} / n_{q}$ of protons falls below that of the mesons for $\mathrm{KE}_{T} / n_{q} \geq 1 \mathrm{GeV}$. This is the range where the proton $v_{2}\left(p_{T}\right)$ begins falling in noncentral collisions but remains relatively constant in central collisions.

On the other hand, it is widely accepted that the relevant scaling variable for quark-recombination is the transverse momentum per quark, since it is the momentum and not the energy that is additive in the recombination models. Therefore, to examine the $n_{q}$ scaling in the recombination regime we show the quark-numberscaled $v_{2}$ as a function of $p_{T} / n_{q}$ in panels (e) and (f) of Fig. 8, For central collisions (Fig. 8(e)), the universal behavior appears to remains valid within the statistical and systematic uncertainties. Since the changes in $v_{2}$ are relatively small at higher $p_{T}$, shifting the $x$-axis from $\mathrm{KE}_{T} / n_{q}$ to $p_{T} / n_{q}$ does not change the shape of the curves significantly. For noncentral collisions (Fig. 8(f)), the proton data are systematically below the pion data at all $p_{T} / n_{q}$, although they are at the edge of the systematic uncertainties for $p_{T} / n_{q} \leq 1.3 \mathrm{GeV} / c$, which corresponds to $\mathrm{KE}_{T} / n_{q} \leq 1 \mathrm{GeV} / c$. We note that despite this systematic offset, the $n_{q}$ scaling makes the shape of the pion and proton curves very similar below the breaking point. Above that point, quark recombination is clearly violated.

Some model calculations [30] have shown that the breaking of $n_{q}$ scaling occurs at the transition between purely thermal and thermal+shower recombination. In the $50-60 \%$ centrality class this can happen for values of $\mathrm{KE}_{T}$ as low as $\mathrm{KE}_{T} / n_{q} \approx 0.5 \mathrm{GeV}$, while in the 0-5\% centrality class this occurs at values as high as $\mathrm{KE}_{T} / n_{q} \approx 1.5 \mathrm{GeV}$. Similar features have been observed in the data presented in this paper. On the other hand, for pions and protons, the nuclear modification factors $\left(R_{\mathrm{AA}}\right)$, which are used to quantify the amount of partonic energy loss in the medium, have been found to be consistent with each other for $p_{T}>5 \mathrm{GeV} / c$ [46, 49 51]. This indicates that a simple interplay between recombination and jet energy loss is not enough to explain the $v_{2}$ and $R_{\mathrm{AA}}$ of pions and protons in $\mathrm{Au}+\mathrm{Au}$ collisions in this $p_{T}$ region. Additional considerations may include the nonAbelian nature of jet energy loss [52], the quark versus gluon fragmentation production of pions and protons [53 55], and jet chemistry effects such as enhanced parton splitting [56] and jet conversion [57]. Detailed model calculations that take all of these effects into account are not yet available, and it is an open question whether doing so is enough for an adequate interpretation of the $p_{T} v_{2}$ and $R_{\mathrm{AA}}$ of pions and protons.

To further investigate the centrality dependence of the $n_{q}$ scaling breaking, results with finer centrality bins are shown in Fig. 9. The quark number scaled $v_{2}\left(v_{2} / n_{q}\right)$ of pions, kaons, and protons are shown as a function of the kinetic energy per quark $\mathrm{KE}_{T} / n_{q}$ in $0-10 \%$ (panel (a)), 10-20\% (panel (b)), 20-40\% (panel (c)), and 40-60\% centrality (panel $(\mathrm{d})$ ). The error bars (shaded boxes) represent the statistical (systematic) uncertainties. The systematic uncertainties shown are type A and B only. Not shown are the type $\mathrm{C}$ systematic uncertainties, which are from the event-plane resolution, geometrical acceptance, and run-by-run dependence are around 10.5\% (3.5\%) for 0-10\% (40-60\%). These results with finer centrality bins show that the breaking of $n_{q}$ scaling has a clear centrality dependence.

We also compare our results with the existing $v_{2}$ results for $K_{S}^{0}$ and $\Lambda$ as measured by the STAR collaboration using the event-plane method [21] in the $0-10 \%$ and $10-40 \%$ centrality classes, which are shown in panel (a) and panel (b) of Fig. 10, respectively. Since the event-plane and particles are measured in the same rapidity gap by the STAR detector in their event-plane method, the $v_{2}$ values from STAR measurements are expected to be systematically larger than those measured by PHENIX [21, 47] due to nonflow effects. In the $0-10 \%$ centrality class, the $v_{2}$ of pions and protons in this study are systematically lower than the $v_{2}$ of $K_{S}^{0}$ and $\Lambda$ by $17 \%$ independent of $p_{T}$, but they are within the systematic uncertainties. The $n_{q}$ scaling appears to hold in this centrality class for each particle species. In the 10-40\% centrality class, the $v_{2}$ of pions and protons are consistent with that of $K_{S}^{0}$ and $\Lambda$ in the overlapping $\mathrm{KE}_{T}$ region. While the presence of the scaling breaking is not clear in the $K_{S}^{0}$ and $\Lambda$ results, the improved precision and extended $\mathrm{KE}_{T}$ reach of the present study unambiguously demonstrates the breaking of $n_{q}$ scaling in this centrality class. 


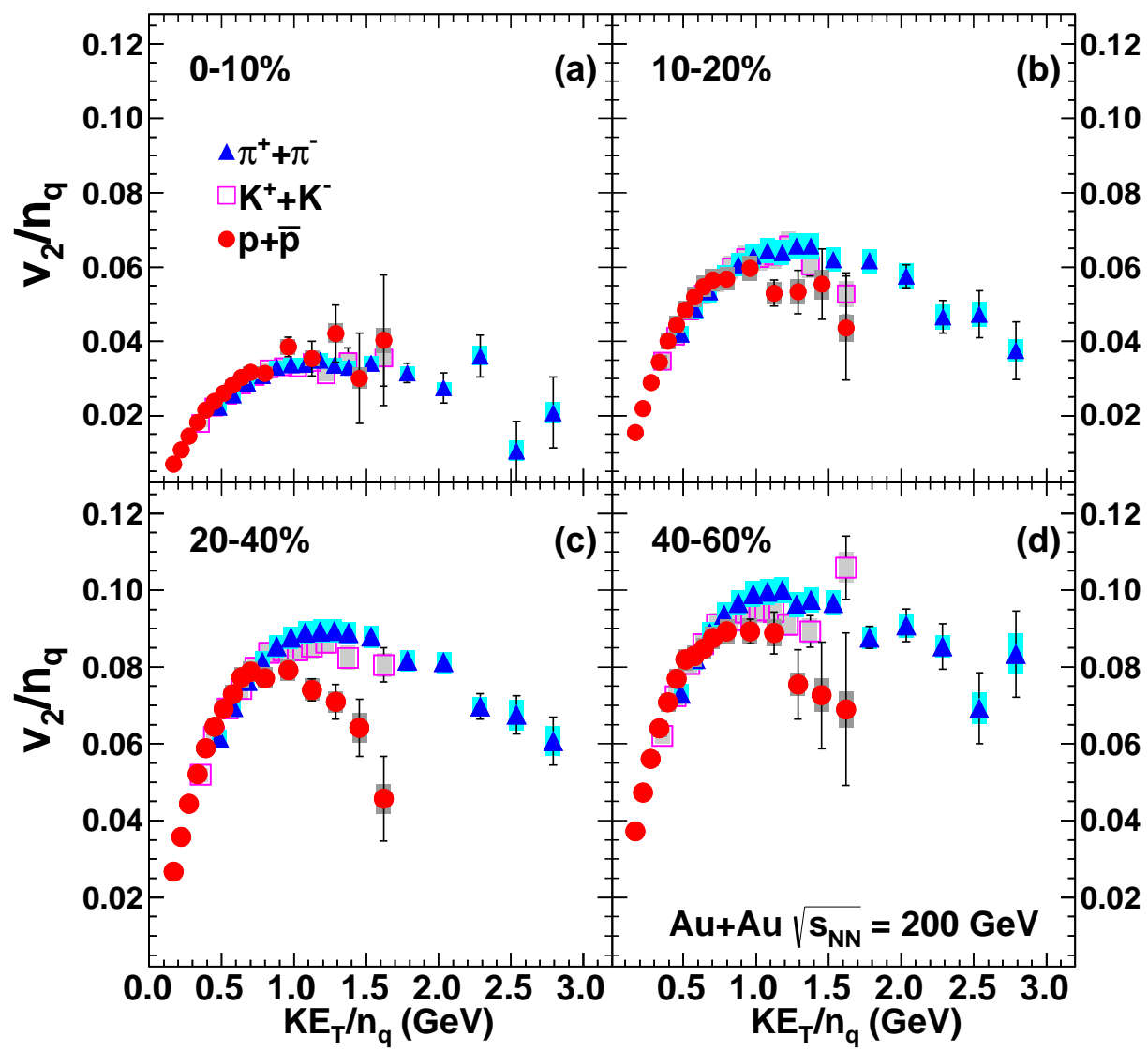

FIG. 9: (color online) The quark-number-scaled $v_{2}\left(v_{2} / n_{q}\right)$ of identified hadrons are shown as a function of the kinetic energy per quark, $\mathrm{KE}_{T} / n_{q}$ in 0-10\% centrality (panel (a)), 10-20\% (panel (b)), 20-40\% (panel (c)), and 40-60\% centrality (panel (d)) in $\mathrm{Au}+\mathrm{Au}$ collisions at $\sqrt{s_{N N}}=200 \mathrm{GeV}$. The error bars (shaded boxes) represent the statistical (systematic) uncertainties. The systematic uncertainties shown are type A and B only.

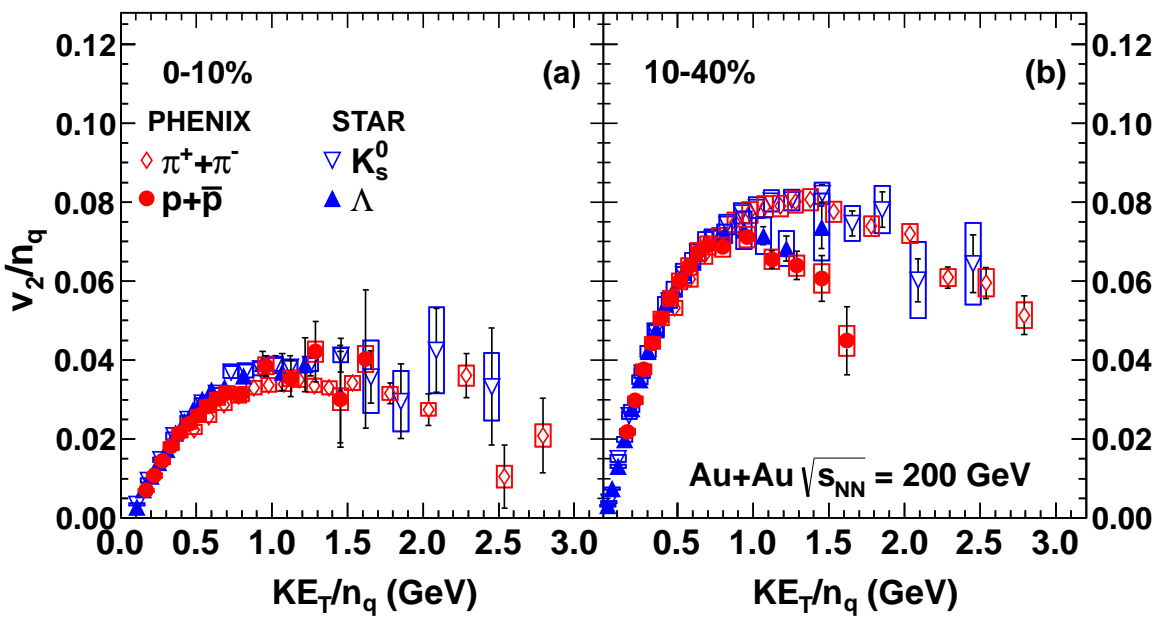

FIG. 10: (color online) The quark-number-scaled $v_{2}\left(v_{2} / n_{q}\right)$ of identified hadrons are shown as a function of the kinetic energy per quark, $\mathrm{KE}_{T} / n_{q}$ in 0-10\% centrality (panel (a)) and 10-40\% centrality (panel (b)) in Au+Au collisions at $\sqrt{s_{N N}}=200 \mathrm{GeV}$. The $v_{2}$ of $\Lambda$ and $K_{S}^{0}$ are measured by STAR collaboration [21]. The error bars (open boxes) represent the statistical (systematic) uncertainties. The systematic uncertainties shown on the results from this study are type A and B only. 


\section{SUMMARY}

We have presented a high-statistics study of baryon and meson azimuthal anisotropy $v_{2}$ measured up to $p_{T}$ of $6 \mathrm{GeV} / c$ as a function of centrality in $\sqrt{s_{N N}}=200 \mathrm{GeV}$ $\mathrm{Au}+\mathrm{Au}$ collisions. The $n_{q}$ scaling is found to exhibit strong dependence on the collision centrality. Significant deviations from $n_{q}$ scaling are found in noncentral collisions, starting from the 10-20\% centrality class, as $\mathrm{KE}_{T} / n_{q}>0.7 \mathrm{GeV}$. These results indicate that parton fragmentation and the associated energy loss may play an important role in generating the azimuthal anisotropy of particle emission. Conversely, in central collisions, such as the $0-10 \%$ centrality class, the universal $n_{q}$ scaling appears to hold to $\mathrm{KE}_{T} / n_{q}=1.5 \mathrm{GeV}$, supporting parton recombination as the dominant mode of particle production at intermediate transverse momentum in central $\mathrm{Au}+\mathrm{Au}$ collisions at top RHIC energy.

\section{ACKNOWLEDGMENTS}

We thank the staff of the Collider-Accelerator and Physics Departments at Brookhaven National Laboratory and the staff of the other PHENIX participating institutions for their vital contributions. We acknowledge support from the Office of Nuclear Physics in the Office of Science of the Department of Energy, the National Science Foundation, Abilene Christian University
Research Council, Research Foundation of SUNY, and Dean of the College of Arts and Sciences, Vanderbilt University (U.S.A), Ministry of Education, Culture, Sports, Science, and Technology and the Japan Society for the Promotion of Science (Japan), Conselho Nacional de Desenvolvimento Científico e Tecnológico and Fundação de Amparo à Pesquisa do Estado de São Paulo (Brazil), Natural Science Foundation of China (P. R. China), Ministry of Education, Youth and Sports (Czech Republic), Centre National de la Recherche Scientifique, Commissariat à l'Énergie Atomique, and Institut National de Physique Nucléaire et de Physique des Particules (France), Ministry of Industry, Science and Tekhnologies, Bundesministerium für Bildung und Forschung, Deutscher Akademischer Austausch Dienst, and Alexander von Humboldt Stiftung (Germany), Hungarian National Science Fund, OTKA (Hungary), Department of Atomic Energy and Department of Science and Technology (India), Israel Science Foundation (Israel), National Research Foundation and WCU program of the Ministry Education Science and Technology (Korea), Ministry of Education and Science, Russian Academy of Sciences, Federal Agency of Atomic Energy (Russia), VR and the Wallenberg Foundation (Sweden), the U.S. Civilian Research and Development Foundation for the Independent States of the Former Soviet Union, the US-Hungarian Fulbright Foundation for Educational Exchange, and the US-Israel Binational Science Foundation.
[1] K. Adcox et al. (PHENIX Collaboration), Nucl. Phys. A 757, 184 (2005).

[2] J. Adams et al. (STAR Collaboration), Nucl. Phys. A 757, 102 (2005).

[3] B. B. Back et al. (PHOBOS Collaboration), Nucl. Phys. A 757, 28 (2005).

[4] I. Arsene et al. (BRAHMS Collaboration), Nucl. Phys. A 757, 1 (2005).

[5] E. V. Shuryak, Nucl. Phys. A 750, 64 (2005).

[6] M. Gyulassy and L. McLerran, Nucl. Phys. A 750, 30 (2005).

[7] U. W. Heinz, arXiv:0901.4355 (2009).

[8] P. Romatschke, Int. J. Mod. Phys. E 19, 1 (2010).

[9] D. A. Teaney, arXiv:0905.2433 (2009).

[10] P. K. Kovtun, D. T. Son, and A. O. Starinets, Phys. Rev. Lett. 94, 111601 (2005).

[11] S. S. Adler et al. (PHENIX Collaboration), Phys. Rev. C 76, 034904 (2007).

[12] S. Afanasiev et al. (PHENIX Collaboration), Phys. Rev. C 80, 054907 (2009).

[13] A. Adare et al. (PHENIX Collaboration), Phys. Rev. Lett. 105, 142301 (2010).

[14] S. A. Bass, C. Gale, A. Majumder, C. Nonaka, G.-Y. Qin, et al., Phys. Rev. C 79, 024901 (2009).

[15] X. N. Wang, Phys. Rev. C 63, 054902 (2001).

[16] S. S. Adler et al. (PHENIX Collaboration), Phys. Rev. Lett. 91, 182301 (2003).
[17] J. Adams et al. (STAR Collaboration), Phys. Rev. Lett. 92, 052302 (2004).

[18] A. Adare et al. (PHENIX Collaboration), Phys. Rev. Lett. 98, 162301 (2007).

[19] S. Afanasiev et al. (PHENIX Collaboration), Phys. Rev. Lett. 99, 052301 (2007).

[20] B. Abelev et al. (the STAR Collaboration), Phys. Rev. C 75, 054906 (2007).

[21] B. Abelev et al. (STAR Collaboration), Phys. Rev. C 77, 054901 (2008).

[22] R. C. Hwa and C. B. Yang, Phys. Rev. C 67, 034902 (2003).

[23] R. J. Fries, B. Muller, C. Nonaka, and S. A. Bass, Phys. Rev. Lett. 90, 202303 (2003).

[24] R. J. Fries, B. Muller, C. Nonaka, and S. A. Bass, Phys. Rev. C 68, 044902 (2003).

[25] V. Greco, C. M. Ko, and P. Levai, Phys. Rev. Lett. 90, 202302 (2003).

[26] D. Molnar and S. A. Voloshin, Phys. Rev. Lett. 91, 092301 (2003).

[27] S. S. Adler et al. (PHENIX Collaboration), Phys. Rev. Lett. 91, 172301 (2003).

[28] S. S. Adler et al. (PHENIX Collaboration), Phys. Rev. C 69, 034909 (2004).

[29] B. Muller, R. J. Fries, and S. A. Bass, Phys. Lett. B 618, 77 (2005).

[30] C. B. Chiu, R. C. Hwa, and C. B. Yang, Phys. Rev. C 
78, 044903 (2008).

[31] G. Ferini, M. Colonna, M. Di Toro, and V. Greco, Phys. Lett. B 670, 325 (2009).

[32] K. Dusling, G. D. Moore, and D. Teaney, Phys. Rev. C 81, 034907 (2010).

[33] R. A. Lacey, A. Taranenko, R. Wei, N. Ajitanand, J. Alexander, et al., Phys. Rev. C 82, 034910 (2010).

[34] O. Fochler, Z. Xu, and C. Greiner, Phys. Rev. Lett. 102, 202301 (2009).

[35] R. A. Lacey, N. Ajitanand, J. Alexander, X. Gong, J. Jia, et al., Phys. Rev. C 80, 051901 (2009).

[36] T. Hirano and Y. Nara, Phys. Rev. C 69, 034908 (2004).

[37] J. C. Dunlop, M. A. Lisa, and P. Sorensen, Phys. Rev. C 84, 044914 (2011).

[38] K. Adcox et al. (PHENIX Collaboration), Nucl. Instrum. Meth. A 499, 469 (2003).

[39] E. Richardson et al. (PHENIX Collaboration), Nucl. Instrum. Meth. A 636, 99 (2011).

[40] A. M. Poskanzer and S. A. Voloshin, Phys. Rev. C 58, 1671 (1998).

[41] M. Chiu (PHENIX Collaboration), AIP Conf. Proc. 915, 539 (2007).

[42] B. Bonner et al., Nucl. Instr. Meth. A 508, 181 (2003).

[43] W. J. Llope et al., Nucl. Instrum. Meth. A 596, 430 (2008).

[44] J. Adams et al. (STAR Collaboration), Phys. Rev. Lett.
92, 052302 (2004).

[45] J. Adams et al., (STAR Collaboration), nucl-ex/0601042 (2006).

[46] G. Agakishiev et al., (STAR Collaboration), arXiv:1110.0579 (2011).

[47] S. S. Adler et al. (PHENIX Collaboration), Phys. Rev. Lett. 96, 032302 (2006).

[48] S. Afanasiev et al. (PHENIX Collaboration), Phys. Rev. C 80, 024909 (2009).

[49] B. I. Abelev et al. (STAR Collaboration), Phys. Rev. Lett. 97, 152301 (2006).

[50] A. Adare et al. (PHENIX Collaboration), Phys. Rev. C 83, 064903 (2011).

[51] R. Belmont (PHENIX Collaboration), Nucl. Phys. A 830, 697c (2009).

[52] X. N. Wang, Phys. Rev. C 58, 2321 (1998).

[53] S. Albino, B. A. Kniehl, and G. Kramer, Nucl. Phys. B 725, 181 (2005).

[54] S. Albino, B. A. Kniehl, and G. Kramer, Nucl. Phys. B 803, 42 (2008).

[55] D. de Florian, R. Sassot, and M. Stratmann, Phys. Rev. D 76, 074033 (2007).

[56] S. Sapeta and U. A. Wiedemann, Eur. Phys. J. C55, 293 (2008).

[57] W. Liu and R. J. Fries, Phys. Rev. C 77, 054902 (2008). 
published in Phys. Rev. C 88, 024906 (2013)

\section{Spectra and ratios of identified particles in $\mathbf{A u}+\mathbf{A u}$ and $d+\mathbf{A u}$ collisions at $\sqrt{s_{N N}}=200 \mathrm{GeV}$}

A. Adare, ${ }^{13}$ S. Afanasiev, ${ }^{30}$ C. Aidala,${ }^{43,}{ }^{44}$ N.N. Ajitanand,${ }^{62}$ Y. Akiba,${ }^{56,57}$ H. Al-Bataineh, ${ }^{50}$ J. Alexander, ${ }^{62}$ A. Angerami, ${ }^{14}$ K. Aoki,${ }^{35,}{ }^{56}$ N. Apadula, ${ }^{63}$ Y. Aramaki, ${ }^{12,}{ }^{56}$ E.T. Atomssa, ${ }^{36}$ R. Averbeck, ${ }^{63}$ T.C. Awes, ${ }^{52}$ B. Azmoun,${ }^{7}$ V. Babintsev, ${ }^{24}$ M. Bai,${ }^{6}$ G. Baksay,${ }^{20}$ L. Baksay,${ }^{20}$ K.N. Barish,${ }^{8}$ B. Bassalleck ${ }^{49}$ A.T. Basye,${ }^{1}$ S. Bathe,${ }^{5,8,57}$ V. Baublis, ${ }^{55}$ C. Baumann ${ }^{45}$ A. Bazilevsky, ${ }^{7}$ S. Belikov, ${ }^{7},{ }^{6}$ R. Belmont,${ }^{44,67}$ R. Bennett, ${ }^{63}$

A. Berdnikov, ${ }^{59}$ Y. Berdnikov, ${ }^{59}$ J.H. Bhom, ${ }^{71}$ A.A. Bickley ${ }^{13}$ D.S. Blau, ${ }^{34}$ J.S. Bok,${ }^{71}$ K. Boyle, ${ }^{63}$ M.L. Brooks, ${ }^{39}$

H. Buesching, ${ }^{7}$ V. Bumazhnov, ${ }^{24}$ G. Bunce,,${ }^{7} 57$ S. Butsyk, ${ }^{39}$ C.M. Camacho, ${ }^{39}$ S. Campbell, ${ }^{63}$ A. Caringi, ${ }^{46}$ C.-H. Chen, ${ }^{63}$ C.Y. Chi,${ }^{14}$ M. Chiu,${ }^{7}$ I.J. Choi,${ }^{71}$ J.B. Choi,${ }^{10}$ R.K. Choudhury,${ }^{4}$ P. Christiansen, ${ }^{41}$

T. Chujo ${ }^{66}$ P. Chung, ${ }^{62}$ O. Chvala, ${ }^{8}$ V. Cianciolo, ${ }^{52}$ Z. Citron, ${ }^{63}$ B.A. Cole,${ }^{14}$ Z. Conesa del Valle, ${ }^{36}$ M. Connors, ${ }^{63}$ P. Constantin, ${ }^{39}$ M. Csanád, ${ }^{18}$ T. Csörgö,${ }^{70}$ T. Dahms, ${ }^{63}$ S. Dairaku ${ }^{35,56}$ I. Danchev, ${ }^{67}$ K. Das, ${ }^{21}$ A. Datta, ${ }^{43}$ G. David, ${ }^{7}$ M.K. Dayananda, ${ }^{22}$ A. Denisov, ${ }^{24}$ A. Deshpande, ${ }^{57,}{ }^{63}$ E.J. Desmond, ${ }^{7}$ K.V. Dharmawardane,${ }^{50}$ O. Dietzsch,${ }^{60}$ A. Dion,${ }^{28,63}$ M. Donadelli, ${ }^{60}$ O. Drapier, ${ }^{36}$ A. Drees, ${ }^{63}$ K.A. Drees,${ }^{6}$ J.M. Durham, ${ }^{39,63}$ A. Durum, ${ }^{24}$ D. Dutta,${ }^{4}$ L. D'Orazio, ${ }^{42}$ S. Edwards,${ }^{21}$ Y.V. Efremenko, ${ }^{52}$ F. Ellinghaus ${ }^{13}$

T. Engelmore ${ }^{14}$ A. Enokizono, ${ }^{38,52}$ H. En'yo, ${ }^{56,57}$ S. Esumi ${ }^{66}$ B. Fadem,${ }^{46}$ D.E. Fields, ${ }^{49}$ M. Finger, ${ }^{9}$ M. Finger, Jr. ${ }^{9}$ F. Fleuret, ${ }^{36}$ S.L. Fokin, ${ }^{34}$ Z. Fraenkel, ${ }^{69}$, J.E. Frantz, ${ }^{51,}{ }^{63}$ A. Franz,${ }^{7}$ A.D. Frawley, ${ }^{21}$ K. Fujiwara, ${ }^{56}$ Y. Fukao, ${ }^{56}$ T. Fusayasu, ${ }^{48}$ I. Garishvili, ${ }^{64}$ A. Glenn, ${ }^{13,}{ }^{38}$ H. Gong,,${ }^{63}$ M. Gonin, ${ }^{36}$ Y. Goto, ${ }^{56,57}$ R. Granier de Cassagnac, ${ }^{36}$ N. Grau ${ }^{2,}{ }^{14}$ S.V. Greene, ${ }^{67}$ G. Grim,${ }^{39}$ M. Grosse Perdekamp,${ }^{25,}{ }^{57}$ T. Gunji ${ }^{12}$

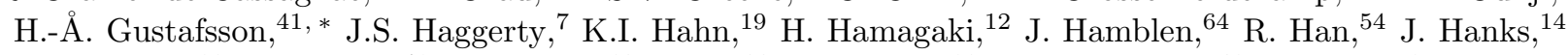
E.P. Hartouni, ${ }^{38}$ E. Haslum,${ }^{41}$ R. Hayano, ${ }^{12}$ X. He, ${ }^{22}$ M. Heffner, ${ }^{38}$ T.K. Hemmick, ${ }^{63}$ T. Hester, ${ }^{8}$ J.C. Hill, ${ }^{28}$ M. Hohlmann, ${ }^{20}$ W. Holzmann, ${ }^{14}$ K. Homma,${ }^{23}$ B. Hong, ${ }^{33}$ T. Horaguchi, ${ }^{23}$ D. Hornback, ${ }^{64}$ S. Huang, ${ }^{67}$ T. Ichihara, ${ }^{56,57}$ R. Ichimiya, ${ }^{56}$ J. Ide ${ }^{46}$ Y. Ikeda, ${ }^{66}$ K. Imai, ${ }^{29,}{ }^{35,}{ }^{56}$ M. Inaba, ${ }^{66}$ D. Isenhower, ${ }^{1}$ M. Ishihara, ${ }^{56}$ T. Isobe, ${ }^{12,56}$ M. Issah, ${ }^{67}$ A. Isupov, ${ }^{30}$ D. Ivanischev, ${ }^{55}$ Y. Iwanaga, ${ }^{23}$ B.V. Jacak,${ }^{63}$ J. Jia,${ }^{7,62}$ X. Jiang, ${ }^{39}$ J. Jin, ${ }^{14}$ B.M. Johnson, ${ }^{7}$ T. Jones, ${ }^{1}$ K.S. Joo, ${ }^{47}$ D. Jouan, ${ }^{53}$ D.S. Jumper,${ }^{1}$ F. Kajihara, ${ }^{12}$ S. Kametani, ${ }^{56}$ N. Kamihara, ${ }^{57}$ J. Kamin, ${ }^{63}$ J.H. Kang, ${ }^{71}$ J. Kapustinsky, ${ }^{39}$ K. Karatsu, ${ }^{35,}{ }^{56}$ M. Kasai, ${ }^{56,58}$ D. Kawall, ${ }^{43,57}$ M. Kawashima, ${ }^{56,58}$ A.V. Kazantsev, ${ }^{34}$ T. Kempel, ${ }^{28}$ A. Khanzadeev, ${ }^{55}$ K.M. Kijima, ${ }^{23}$ J. Kikuchi, ${ }^{68}$ A. Kim, ${ }^{19}$ B.I. Kim,${ }^{33}$ D.H. Kim,${ }^{47}$ D.J. Kim,${ }^{31}$ E. Kim ${ }^{61}$ E.-J. Kim,${ }^{10}$ S.H. Kim,${ }^{71}$ Y.-J. Kim,${ }^{25}$ Y.J. Kim,${ }^{25}$ E. Kinney, ${ }^{13}$ K. Kiriluk, ${ }^{13}$ Á. Kiss, ${ }^{18}$ E. Kistenev,${ }^{7}$ D. Kleinjan, ${ }^{8}$ L. Kochenda, ${ }^{55}$ B. Komkov, ${ }^{55}$ M. Konno, ${ }^{66}$ J. Koster, ${ }^{25}$ D. Kotchetkov ${ }^{49}$ A. Kozlov, ${ }^{69}$ A. Král, ${ }^{15}$ A. Kravitz, ${ }^{14}$ G.J. Kunde, ${ }^{39}$ K. Kurita, ${ }^{56,}{ }^{58}$ M. Kurosawa, ${ }^{56}$ Y. Kwon, ${ }^{71}$ G.S. Kyle ${ }^{50}$ R. Lacey, ${ }^{62}$ Y.S. Lai, ${ }^{14}$ J.G. Lajoie ${ }^{28}$ A. Lebedev ${ }^{28}$ D.M. Lee,${ }^{39}$ J. Lee, ${ }^{19}$ K. Lee ${ }^{61}$ K.B. Lee, ${ }^{33}$ K.S. Lee, ${ }^{33}$ M.J. Leitch, ${ }^{39}$ M.A.L. Leite, ${ }^{60}$ E. Leitner, ${ }^{67}$ B. Lenzi, ${ }^{60}$ X. Li,${ }^{11}$ P. Lichtenwalner, ${ }^{46}$ P. Liebing, ${ }^{57}$ L.A. Linden Levy, ${ }^{13}$ T. Liška, ${ }^{15}$ A. Litvinenko, ${ }^{30}$ H. Liu, ${ }^{39,}{ }^{50}$ M.X. Liu, ${ }^{39}$ B. Love,${ }^{67}$ R. Luechtenborg, ${ }^{45}$ D. Lynch, ${ }^{7}$

C.F. Maguire, ${ }^{67}$ Y.I. Makdisi, ${ }^{6}$ A. Malakhov,${ }^{30}$ M.D. Malik, ${ }^{49}$ V.I. Manko, ${ }^{34}$ E. Mannel, ${ }^{14}$ Y. Mao, ${ }^{54,56}$

H. Masui ${ }^{66}$ F. Matathias, ${ }^{14}$ M. McCumber ${ }^{63}$ P.L. McGaughey ${ }^{39}$ D. McGlinchey, ${ }^{13,}{ }^{21}$ N. Means,${ }^{63}$ B. Meredith, ${ }^{25}$

Y. Miake ${ }^{66}$ T. Mibe ${ }^{32}$ A.C. Mignerey, ${ }^{42}$ P. Mikeš,${ }^{9}{ }^{27}$ K. Miki, ${ }^{56,66}$ A. Milov, ${ }^{7}$ M. Mishra, ${ }^{3}$ J.T. Mitchell, ${ }^{7}$ A.K. Mohanty ${ }^{4}$ H.J. Moon, ${ }^{47}$ Y. Morino, ${ }^{12}$ A. Morreale, ${ }^{8}$ D.P. Morrison,, , , T.V. Moukhanova, ${ }^{34}$ T. Murakami, ${ }^{35}$ J. Murata, ${ }^{56,58}$ S. Nagamiya, ${ }^{32}$ J.L. Nagle, ${ }^{13}$, 团 M. Naglis, ${ }^{69}$ M.I. Nagy, ${ }^{18,70}$ I. Nakagawa, ${ }^{56,57}$ Y. Nakamiya, ${ }^{23}$ K.R. Nakamura, ${ }^{35,56}$ T. Nakamura, ${ }^{23,32,56}$ K. Nakano, ${ }^{56,65}$ S. Nam, ${ }^{19}$ J. Newby ${ }^{38}$ M. Nguyen, ${ }^{63}$ M. Nihashi, ${ }^{23}$

R. Nouicer, ${ }^{7}$ A.S. Nyanin, ${ }^{34}$ C. Oakley, ${ }^{22}$ E. O'Brien, ${ }^{7}$ S.X. Oda,${ }^{12}$ C.A. Ogilvie,${ }^{28}$ M. Oka, ${ }^{66}$ K. Okada, ${ }^{57}$

Y. Onuki, ${ }^{56}$ A. Oskarsson, ${ }^{41}$ M. Ouchida,${ }^{23,}{ }^{56}$ K. Ozawa, ${ }^{12}$ R. Pak,${ }^{7}$ V. Pantuev,${ }^{26,63}$ V. Papavassiliou, ${ }^{50}$ I.H. Park,${ }^{19}$ J. Park, ${ }^{61}$ S.K. Park,${ }^{33}$ W.J. Park,${ }^{33}$ S.F. Pate, ${ }^{50}$ H. Pei, ${ }^{28}$ J.-C. Peng, ${ }^{25}$ H. Pereira, ${ }^{16}$ V. Peresedov ${ }^{30}$ D.Yu. Peressounko, ${ }^{34}$ R. Petti, ${ }^{63}$ C. Pinkenburg, ${ }^{7}$ R.P. Pisani, ${ }^{7}$ M. Proissl,${ }^{63}$ M.L. Purschke,${ }^{7}$ A.K. Purwar, ${ }^{39}$ H. Qu ${ }^{22}$ J. Rak, ${ }^{31}$ A. Rakotozafindrabe, ${ }^{36}$ I. Ravinovich ${ }^{69}$ K.F. Read,${ }^{52,}{ }^{64}$ S. Rembeczki, ${ }^{20}$ K. Reygers, ${ }^{45}$ V. Riabov ${ }^{55}$ Y. Riabov, ${ }^{55}$ E. Richardson ${ }^{42}$ D. Roach ${ }^{67}$ G. Roche ${ }^{40}$ S.D. Rolnick, ${ }^{8}$ M. Rosati, ${ }^{28}$ C.A. Rosen,${ }^{13}$ S.S.E. Rosendahl ${ }^{41}$ P. Rosnet, ${ }^{40}$ P. Rukoyatkin, ${ }^{30}$ P. Ružička, ${ }^{27}$ B. Sahlmueller, ${ }^{45}{ }^{63}$ N. Saito, ${ }^{32}$ T. Sakaguchi, ${ }^{7}$ K. Sakashita, ${ }^{56,65}$ V. Samsonov, ${ }^{55}$ S. Sano, ${ }^{12,68}$ T. Sato ${ }^{66}$ S. Sawada, ${ }^{32}$ K. Sedgwick, ${ }^{8}$ J. Seele, ${ }^{13}$ R. Seidl,,${ }^{25,} 57$ A.Yu. Semenov, ${ }^{28}$ R. Seto, ${ }^{8}$ D. Sharma, ${ }^{69}$ I. Shein, ${ }^{24}$ T.-A. Shibata, ${ }^{56,65}$ K. Shigaki, ${ }^{23}$ M. Shimomura, ${ }^{66}$ K. Shoji ${ }^{35,56}$ P. Shukla, ${ }^{4}$ A. Sickles,${ }^{7}$ C.L. Silva, ${ }^{28,60}$ D. Silvermyr, ${ }^{52}$ C. Silvestre ${ }^{16}$ K.S. Sim, ${ }^{33}$ B.K. Singh, ${ }^{3}$

C.P. Singh, ${ }^{3}$ V. Singh,${ }^{3}$ M. Slunečka, ${ }^{9}$ R.A. Soltz,${ }^{38}$ W.E. Sondheim, ${ }^{39}$ S.P. Sorensen,${ }^{64}$ I.V. Sourikova,${ }^{7}$

N.A. Sparks, ${ }^{1}$ P.W. Stankus, ${ }^{52}$ E. Stenlund ${ }^{41}$ S.P. Stoll,${ }^{7}$ T. Sugitate,${ }^{23}$ A. Sukhanov, ${ }^{7}$ J. Sziklai, ${ }^{70}$ E.M. Takagui ${ }^{60}$

A. Taketani, ${ }^{56,57}$ R. Tanabe, ${ }^{66}$ Y. Tanaka, ${ }^{48}$ S. Taneja,${ }^{63}$ K. Tanida,${ }^{35,56,57}$ M.J. Tannenbaum, ${ }^{7}$ S. Tarafdar, ${ }^{3}$

A. Taranenko, ${ }^{62}$ P. Tarján, ${ }^{17}$ H. Themann, ${ }^{63}$ D. Thomas, ${ }^{1}$ T.L. Thomas, ${ }^{49}$ M. Togawa,${ }^{35,56,57}$ A. Toia,${ }^{63}$ 
L. Tomášek, ${ }^{27}$ H. Torii, ${ }^{23}$ R.S. Towell, ${ }^{1}$ I. Tserruya, ${ }^{69}$ Y. Tsuchimoto,${ }^{23}$ C. Vale,,${ }^{7}{ }^{28}$ H. Valle, ${ }^{67}$ H.W. van Hecke, ${ }^{39}$ E. Vazquez-Zambrano, ${ }^{14}$ A. Veicht,${ }^{25}$ J. Velkovska,${ }^{67}$ R. Vértesi, ${ }^{17,70}$ A.A. Vinogradov ${ }^{34}$ M. Virius, ${ }^{15}$ V. Vrba ${ }^{27}$ E. Vznuzdaev ${ }^{55}$ X.R. Wang, ${ }^{50}$ D. Watanabe, ${ }^{23}$ K. Watanabe,${ }^{66}$ Y. Watanabe,${ }^{56,57}$ F. Wei, ${ }^{28}$ R. Wei ${ }^{62}$ J. Wessels, ${ }^{45}$ S.N. White, ${ }^{7}$ D. Winter,${ }^{14}$ J.P. Wood,${ }^{1}$ C.L. Woody,${ }^{7}$ R.M. Wright,${ }^{1}$ M. Wysocki, ${ }^{13}$ W. Xie, ${ }^{57}$ Y.L. Yamaguchi, ${ }^{12}$ K. Yamaura, ${ }^{23}$ R. Yang, ${ }^{25}$ A. Yanovich, ${ }^{24}$ J. Ying, ${ }^{22}$ S. Yokkaichi, ${ }^{56,57}$ Z. You, ${ }^{54}$ G.R. Young, ${ }^{52}$ I. Younus, ${ }^{37}, 49$ I.E. Yushmanov ${ }^{34}$ W.A. Zajc,${ }^{14}$ C. Zhang, ${ }^{52}$ S. Zhou, ${ }^{11}$ and L. Zolin ${ }^{30}$

(PHENIX Collaboration)

${ }^{1}$ Abilene Christian University, Abilene, Texas 79699, USA

${ }^{2}$ Department of Physics, Augustana College, Sioux Falls, South Dakota 57197, USA

${ }^{3}$ Department of Physics, Banaras Hindu University, Varanasi 221005, India ${ }^{4}$ Bhabha Atomic Research Centre, Bombay 400 085, India

${ }^{5}$ Baruch College, City University of New York, New York, New York, 10010 USA

${ }^{6}$ Collider-Accelerator Department, Brookhaven National Laboratory, Upton, New York 11973-5000, USA

${ }^{7}$ Physics Department, Brookhaven National Laboratory, Upton, New York 11973-5000, USA

${ }^{8}$ University of California - Riverside, Riverside, California 92521, USA

${ }^{9}$ Charles University, Ovocný trh 5, Praha 1, 116 36, Prague, Czech Republic

${ }^{10}$ Chonbuk National University, Jeonju, 561-756, Korea

${ }^{11}$ Science and Technology on Nuclear Data Laboratory, China Institute of Atomic Energy, Beijing 102413, P. R. China

${ }^{12}$ Center for Nuclear Study, Graduate School of Science, University of Tokyo, 7-3-1 Hongo, Bunkyo, Tokyo 113-0033, Japan

${ }^{13}$ University of Colorado, Boulder, Colorado 80309, USA

${ }^{14}$ Columbia University, New York, New York 10027 and Nevis Laboratories, Irvington, New York 10533, USA

${ }^{15}$ Czech Technical University, Zikova 4, 16636 Prague 6, Czech Republic

${ }^{16}$ Dapnia, CEA Saclay, F-91191, Gif-sur-Yvette, France

${ }^{17}$ Debrecen University, H-4010 Debrecen, Egyetem tér 1, Hungary

${ }^{18}$ ELTE, Eötvös Loránd University, H - 1117 Budapest, Pázmány P. s. 1/A, Hungary

${ }^{19}$ Ewha Womans University, Seoul 120-750, Korea

${ }^{20}$ Florida Institute of Technology, Melbourne, Florida 32901, USA

${ }^{21}$ Florida State University, Tallahassee, Florida 32306, USA

${ }^{22}$ Georgia State University, Atlanta, Georgia 30303, USA

${ }^{23}$ Hiroshima University, Kagamiyama, Higashi-Hiroshima 739-8526, Japan

${ }^{24}$ IHEP Protvino, State Research Center of Russian Federation, Institute for High Energy Physics, Protvino, 142281, Russia

${ }^{25}$ University of Illinois at Urbana-Champaign, Urbana, Illinois 61801, USA

${ }^{26}$ Institute for Nuclear Research of the Russian Academy of Sciences, prospekt 60-letiya Oktyabrya 7a, Moscow 117312, Russia

${ }^{27}$ Institute of Physics, Academy of Sciences of the Czech Republic, Na Slovance 2, 18221 Prague 8, Czech Republic

${ }^{28}$ Iowa State University, Ames, Iowa 50011, USA

${ }^{29}$ Advanced Science Research Center, Japan Atomic Energy Agency, 2-4

Shirakata Shirane, Tokai-mura, Naka-gun, Ibaraki-ken 319-1195, Japan

${ }^{30}$ Joint Institute for Nuclear Research, 141980 Dubna, Moscow Region, Russia

${ }^{31}$ Helsinki Institute of Physics and University of Jyväskylä, P.O.Box 35, FI-40014 Jyväskylä, Finland

${ }^{32}$ KEK, High Energy Accelerator Research Organization, Tsukuba, Ibaraki 305-0801, Japan

${ }^{33}$ Korea University, Seoul, 136-701, Korea

${ }^{34}$ Russian Research Center "Kurchatov Institute", Moscow, 123098 Russia

${ }^{35}$ Kyoto University, Kyoto 606-8502, Japan

${ }^{36}$ Laboratoire Leprince-Ringuet, Ecole Polytechnique, CNRS-IN2P3, Route de Saclay, F-91128, Palaiseau, France

${ }^{37}$ Physics Department, Lahore University of Management Sciences, Lahore, Pakistan

${ }^{38}$ Lawrence Livermore National Laboratory, Livermore, California 94550, USA

${ }^{39}$ Los Alamos National Laboratory, Los Alamos, New Mexico 87545, USA

${ }^{40}$ LPC, Université Blaise Pascal, CNRS-IN2P3, Clermont-Fd, 63177 Aubiere Cedex, France

${ }^{41}$ Department of Physics, Lund University, Box 118, SE-221 00 Lund, Sweden

$4^{42}$ University of Maryland, College Park, Maryland 20742, USA

${ }^{43}$ Department of Physics, University of Massachusetts, Amherst, Massachusetts 01003-9337, USA

${ }^{44}$ Department of Physics, University of Michigan, Ann Arbor, Michigan 48109-1040, USA

${ }^{45}$ Institut fur Kernphysik, University of Muenster, D-48149 Muenster, Germany

${ }^{46}$ Muhlenberg College, Allentown, Pennsylvania 18104-5586, USA

${ }^{47}$ Myongji University, Yongin, Kyonggido 449-728, Korea

${ }^{48}$ Nagasaki Institute of Applied Science, Nagasaki-shi, Nagasaki 851-0193, Japan

${ }^{49}$ University of New Mexico, Albuquerque, New Mexico 87131, USA

${ }^{50}$ New Mexico State University, Las Cruces, New Mexico 88003, USA

${ }^{51}$ Department of Physics and Astronomy, Ohio University, Athens, Ohio 45701, USA

${ }^{52}$ Oak Ridge National Laboratory, Oak Ridge, Tennessee 37831, USA

${ }^{53}$ IPN-Orsay, Universite Paris Sud, CNRS-IN2P3, BP1, F-91406, Orsay, France

${ }^{54}$ Peking University, Beijing 100871, P. R. China 


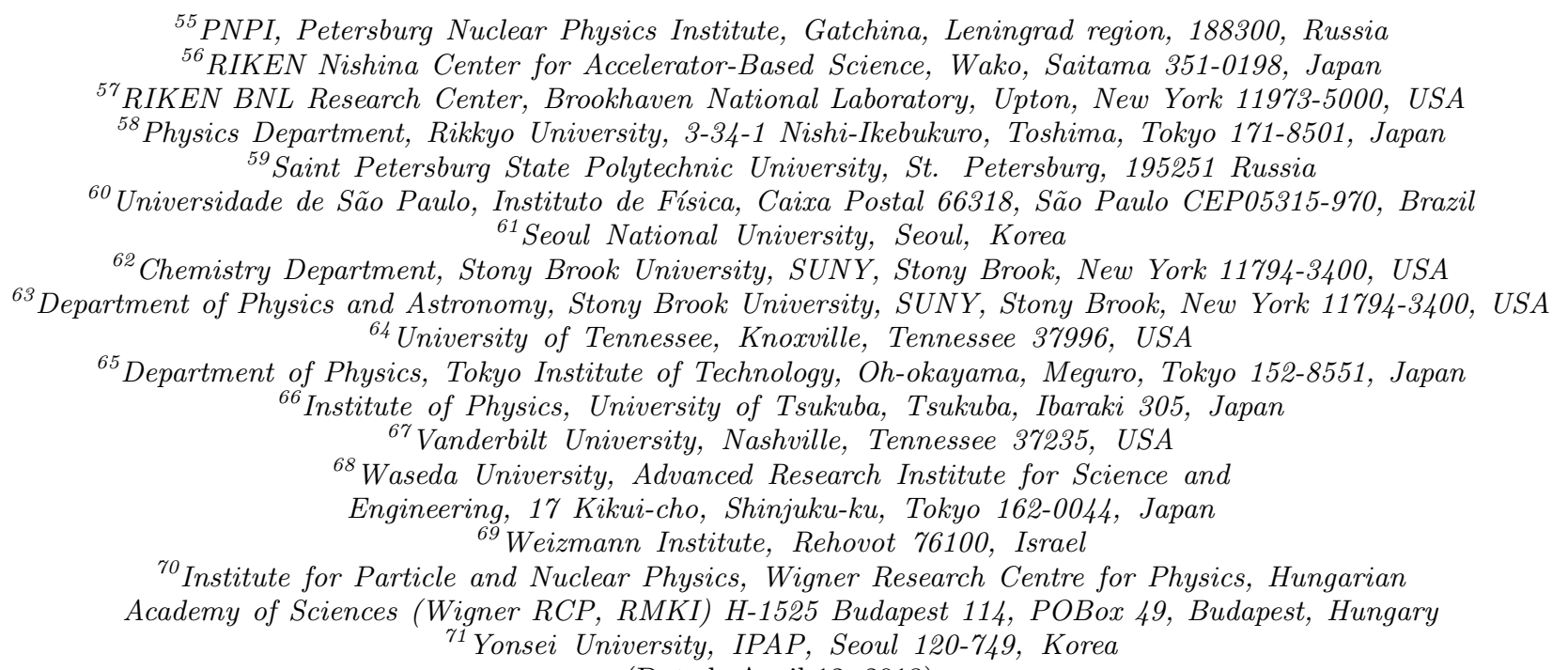

(Dated: April 12, 2013)

The transverse momentum $\left(p_{T}\right)$ spectra and ratios of identified charged hadrons $\left(\pi^{ \pm}, K^{ \pm}, p\right.$, $\bar{p})$ produced in $\sqrt{s_{N N}}=200 \mathrm{GeV} \mathrm{Au}+\mathrm{Au}$ and $\mathrm{d}+\mathrm{Au}$ collisions are reported in five different centrality classes for each collision species. The measurements of pions and protons are reported up to $p_{T}=6 \mathrm{GeV} / \mathrm{c}(5 \mathrm{GeV} / \mathrm{c})$, and the measurements of kaons are reported up to $p_{T}=4 \mathrm{GeV} / \mathrm{c}$ $(3.5 \mathrm{GeV} / \mathrm{c})$ in $\mathrm{Au}+\mathrm{Au}(d+\mathrm{Au})$ collisions. In the intermediate $p_{T}$ region, between $2-5 \mathrm{GeV} / \mathrm{c}, \mathrm{a}$ significant enhancement of baryon to meson ratios compared to those measured in $p+p$ collisions is observed. This enhancement is present in both $\mathrm{Au}+\mathrm{Au}$ and $\mathrm{d}+\mathrm{Au}$ collisions, and increases as the collisions become more central. We compare a class of peripheral $\mathrm{Au}+\mathrm{Au}$ collisions with a class central $\mathrm{d}+\mathrm{Au}$ collisions which have a comparable number of participating nucleons and binary nucleon-nucleon collisions. The $p_{T}$ dependent particle ratios for these classes display a remarkable similarity, which is then discussed.

PACS numbers: 25.75.Dw, 25.75.Ld

\section{INTRODUCTION}

Measurements of identified particles in $\mathrm{Au}+\mathrm{Au}$ collisions allow the study of particle production mechanisms in a hot and dense nuclear medium and probe the properties of the quark-gluon plasma (QGP) 1- $[4]$. In $d+\mathrm{Au}$ collisions, these measurements allow the study of cold nuclear matter effects on particle production, such as the Cronin enhancement [5, 6], nuclear shadowing [7], and gluon saturation [8]. These cold nuclear matter effects are present in $\mathrm{Au}+\mathrm{Au}$ collisions as well, and the study of $d+\mathrm{Au}$ collisions allows us to determine these effects directly and to disentangle them from the effects of the hot and dense nuclear medium.

One of the most intriguing discoveries in the early days of the research program at the Relativistic Heavy Ion Collider was the significantly enhanced baryon production relative to meson production at intermediate transverse momentum $2 \mathrm{GeV} / c<p_{T}<5 \mathrm{GeV} / c$, as evidenced in the large baryon to meson ratios and the significant dif-

\footnotetext{
*Deceased

${ }^{\dagger}$ PHENIX Co-Spokesperson: morrison@bnl.gov

‡PHENIX Co-Spokesperson: jamie.nagle@colorado.edu
}

ferences in the particle suppression patterns measured by the nuclear modification factors 9 11]. Several classes of models were introduced to explain these differences based on different physical phenomena, such as strong radial flow 12, 13, baryon junctions [14, 15], and hadronization through recombination [16 21].

Additionally, the recombination model has been employed to explain the baryon vs. meson difference in the Cronin enhancement observed in $\mathrm{p}+\mathrm{A}$ collisions [22, 23]. Traditional explanations of the Cronin enhancement involve the multiple soft scatterings in the initial state prior to the hard scattering and subsequent fragmentation of the hard scattered parton 24]. This process can naturally explain the deficit of particle production at low $p_{T}$ and enhancement at intermediate $p_{T}$, but does not account for the particle species dependence at Relativistic-Heavy-Ion-Collider energies 25]. Conversely, in the recombination model, the observed enhancement is attributed to final state effects, i.e. the recombination of soft partons from the nuclear medium with hard scattered partons in a jet. For this reason $d+\mathrm{Au}$ collisions represent an excellent testing ground for the recombination model, since hot nuclear matter effects, such as the collective expansion of the medium, are not expected. However, recent results in $d+\mathrm{Au}$ collisions at $200 \mathrm{GeV}$ [26] and $p+\mathrm{Pb}$ collisions at $5.02 \mathrm{TeV}$ 27 29] 
suggest some collective expansion effects may be present in the most central events.

Measurements of strange particles, such as charged kaons, have also been an interesting subject in heavy ion collisions. An enhancement of strangeness production relative to that in $p+p$ collisions has been observed at various colliding energies [30]. This strangeness enhancement is a possible signature of deconfinement, thermalization, and flavor equilibration [31, 32]. In this scenario, strangeness production is dominated by thermal gluon fusion. The measurement of charged kaons in a broad $p_{T}$ range and in different centrality classes is a significant tool to further understand the thermalization of the system and the mechanism of strangeness production.

To address the particle production in both hot and cold QCD matter, a systematic study of identified particles over a broad $p_{T}$ range with a wide selection of centralities in both $\mathrm{Au}+\mathrm{Au}$ and $d+\mathrm{Au}$ collisions is required. In this paper, the spectra, particle ratios, and nuclear modification factors previously reported by PHENIX in $\mathrm{Au}+\mathrm{Au}$ [10] and $d+\mathrm{Au}$ collisions [25] are revisited, extending the $p_{T}$ reach of previous measurements and significantly improving the statistical precision. In Section II we discuss the experimental apparatus and the detector subsystems used in this analysis; in Section III we discuss the analysis method, including event and track selection, and particle identification; in Section IV we discuss the results; and in Section [V] we summarize our findings.

\section{EXPERIMENTAL SETUP}

The PHENIX experiment is a large, general purpose detector with a wide variety of detector subsystems ideally suited to the study of nuclear matter in conditions of extreme temperature and density. PHENIX is composed of global event property detectors, forward and backward rapidity arms (North and South) dedicated to muon measurements, and two central arm spectrometers (East and West) at midrapidity covering pseudorapidity region of $|\eta|<0.35$ for measurements of photons, electrons, and charged hadrons. Detailed descriptions of the various detector subsystems can be found in [33].

Figure 1 gives a schematic diagram of the PHENIX detector for the 2007 configuration; the 2008 configuration is very similar. The upper panel shows the central spectrometer arms, viewed along the beam line facing North. The lower panel shows the two forward rapidity muon arms (North and South) and the global detectors.

The analysis presented herein makes use of the beambeam counters, the tracking system (drift chamber and two layers of pad chambers), the electromagnetic calorimeter, and the time-of-flight detector in the West arm.

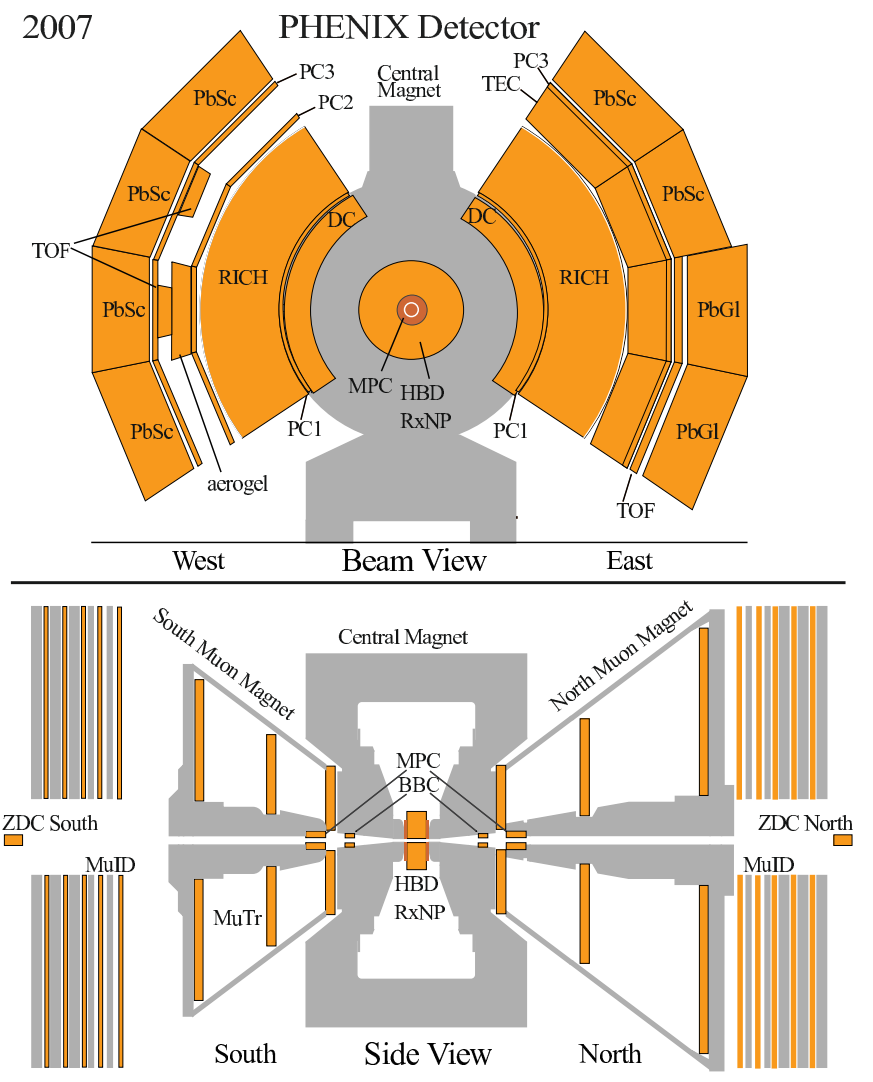

FIG. 1: (Color online) The PHENIX detector configuration for the 2007 data taking period. The 2008 configuration is very similar.

\section{A. Detector subsystems}

The beam-beam counters (BBC) are used for the minimum-bias trigger, the centrality definition, the determination of collision vertex along the beam axis (the $z$-vertex), and the event start time. The BBCs $[34,35$ are located at $\pm 144 \mathrm{~cm}$ from the nominal interaction point. They cover $2 \pi$ in azimuth and a pseudorapidity range of $3.0<|\eta|<3.9$. Each BBC is an array of 64 identical hexagonal detector elements, with the beam pipe passing through the center of the array. Each element is a quartz Cerenkov radiation counter, and the radiator and photomultiplier tube are constructed as a single piece.

The PHENIX tracking system is optimized for the high multiplicity environment of ultra-relativistic heavy ion collisions. It comprises drift chambers (DC) [36] and pad chambers (PC1, PC2, and PC3) [37]. This analysis makes use of the first layer of pad chambers (PC1) and the third layer (PC3). The DCs have an active volume in the radial range $2.02 \mathrm{~m}<r<2.46 \mathrm{~m}$. The $\mathrm{PC} 1$ is mated directly to the back of the DC frame in each arm at a radial distance of $2.49 \mathrm{~m}$. The PC3 is located in each arm at a radial distance of $4.98 \mathrm{~m}$.

The primary particle identification detector used in this analysis is the time-of-flight detector in the West 
half of the central arm spectrometer (TOFW). The TOFW 38, 39] is located at a radial distance of $4.81 \mathrm{~m}$ from the interaction point, having pseudorapidity coverage of $|\eta|<0.35$ and azimuthal coverage of $22^{\circ}$ in two separate sections. The individual elements are multigap resistive plate chambers (MRPCs). Each MRPC has six $230 \mu \mathrm{m}$ gas gaps separated by five $550 \mu \mathrm{m}$ thick glass plates. On each side of the outermost glass plates (1.1 mm thick) are carbon tape electrodes held at $+7 \mathrm{kV}$ on one side and $-7 \mathrm{kV}$ on the other side for a total bias voltage of $14 \mathrm{kV}$. When charged particles traverse the detector, the gas between the plates is ionized and the image charge is collected at each side of the chamber on four copper readout strips. Each strip has dimensions 37 $\times 2.8 \mathrm{~cm}^{2}$ with a separation of $0.3 \mathrm{~cm}$ between them. The strips are oriented lengthwise along the azimuthal direction. Each strip is read out from both top and bottom so that the time difference between them can be used to determine the hit position along the length of the strip with resolution of order $1 \mathrm{~cm}$. The TOFW system is composed of a total of 128 MRPCs, 512 strips, and 1024 readouts. The total timing resolution, which includes the uncertainty in the start time from the BBC, is 84 ps in $\mathrm{Au}+\mathrm{Au}$ collisions [39]. In $d+\mathrm{Au}$ collisions it is $95 \mathrm{ps}$, where the slightly poorer resolution is due to the lower resolution of the start time determination from the BBC. This is due to the lower multiplicity in $d+\mathrm{Au}$ collisions and, for the same reason, the $z$-vertex resolution is also poorer in $d+\mathrm{Au}$ collisions.

\section{ANALYSIS METHOD}

\section{A. Event and Track Selection}

This paper presents an analysis of $\mathrm{Au}+\mathrm{Au}$ collisions at $\sqrt{s_{N N}}=200 \mathrm{GeV}$, collected in 2007, and $d+\mathrm{Au}$ collisions at $\sqrt{s_{N N}}=200 \mathrm{GeV}$, collected in 2008. For each set we select events that pass the minimum-bias trigger, which is defined as a coincidence between the North and South BBCs. In $\mathrm{Au}+\mathrm{Au}$ collisions, this trigger requires two or more photomultiplier tubes firing in each BBC and measures $92 \pm 3 \%$ of the total inelastic cross section; in $d+\mathrm{Au}$ collisions, it requires one or more photomultiplier tubes firing in each BBC and measures $88 \pm 4 \%$ of the total inelastic cross section. We have an additional requirement that the collision vertex is within $|z|<30 \mathrm{~cm}$ of the nominal origin of the coordinate system.

Centrality selection is performed with the BBCs using the Glauber Monte Carlo procedure described in [40], in which the charge in each BBC detector is assumed to be proportional to the number of participating nucleons $N_{\text {part }}$ traveling towards it. For the $\mathrm{Au}+\mathrm{Au}$ system the north and south BBC distributions are summed, but for the $d+\mathrm{Au}$ system, only the south (Au-going) side is used. The BBC charge is assumed to follow a negative binomial distribution (NBD) with a mean of $N_{\text {part }}$ and the remaining NBD parameters determined from a $\chi^{2}$ minimization of the combined Glauber+NBD calculation with respect to the data. The BBC distributions are divided into equal probability bins, and the corresponding Glauber distributions are used to calculate $N_{\text {part }}$ as well as the number of binary nucleon-nucleon collisions $N_{\text {coll }}$, as shown in Table [

TABLE I: Values of $\left\langle N_{\text {coll }}\right\rangle$ and $\left\langle N_{\text {part }}\right\rangle$ for $\mathrm{Au}+\mathrm{Au}$ and $d+\mathrm{Au}$ collisions from Glauber model simulations.

\begin{tabular}{rrr}
\hline \hline Centrality & $\left\langle N_{\text {coll }}\right\rangle$ & $\left\langle N_{\text {part }}\right\rangle$ \\
\hline Au+Au & & \\
$0-10 \%$ & $960.2 \pm 96.1$ & $325.8 \pm 3.8$ \\
$10-20 \%$ & $609.5 \pm 59.8$ & $236.1 \pm 5.5$ \\
$20-40 \%$ & $300.8 \pm 29.6$ & $141.5 \pm 5.8$ \\
$40-60 \%$ & $94.2 \pm 12.0$ & $61.6 \pm 5.1$ \\
$60-92 \%$ & $14.8 \pm 3.0$ & $14.7 \pm 2.9$ \\
\hline$d+\mathrm{Au}$ & & \\
$0-20 \%$ & $15.1 \pm 1.0$ & $15.3 \pm 0.8$ \\
$20-40 \%$ & $6.6 \pm 0.4$ & $11.1 \pm 0.6$ \\
$40-60 \%$ & $3.1 \pm 0.2$ & $7.8 \pm 0.4$ \\
$60-88 \%$ & $7.6 \pm 0.4$ & $4.3 \pm 0.2$ \\
$0-100 \%$ & & $8.5 \pm 0.4$ \\
\hline \hline
\end{tabular}

Charged track reconstruction in the DC is based on a combinatorial Hough transform, which gives the angle in the main bend plane $(r-\phi)$ and thus $p_{T}$. The PC1 is used to determine the hit position in the longitudinal $(z)$ direction. Only tracks with valid information in both the DC and PC1 are used in this analysis. Tracks in $\mathrm{DC} / \mathrm{PC} 1$ are projected to the outer detectors, such as PC3 and TOFW, and matched to hits in those detectors with the minimum distance between the projection and the hit position. The distribution of differences between hits and projections is approximately Gaussian, with an additional underlying background caused by random associations. Only tracks with a difference of less than two standard deviations in both the azimuthal and longitudinal directions in both the PC3 and the TOFW are selected, so as to minimize background contamination. In the $\mathrm{Au}+\mathrm{Au}$ data for $p_{T}>5.0 \mathrm{GeV} / c$, an additional background isolation cut is applied. For these tracks we require $E / p_{T}>0.2$, where $E$ is the energy deposited in the electromagnetic calorimeter. This cut removes low $p_{T}$ particles that are falsely reconstructed as high $p_{T}$ tracks.

\section{B. Particle Identification}

Charged particle identification (PID) is performed by simultaneous measurement of the momentum, time-offlight, and path-length. These quantities are used to determine the mass of the candidate track based on the following relationship: 


$$
m^{2}=\frac{p^{2}}{c^{2}}\left(\frac{t^{2} c^{2}}{L^{2}}-1\right)
$$

where $m$ is the mass, $p$ is the momentum, $c$ is the speed of light, $t$ is the time of flight, and $L$ is the pathlength. The $m^{2}$ distributions are approximately Gaussian. The standard deviation of the distribution $\sigma_{m^{2}}$ can be parametrized as a function of momentum as follows:

$$
\begin{aligned}
\sigma_{m^{2}}^{2} & =\frac{\sigma_{\alpha}^{2}}{K_{1}^{2}}\left(4 m^{4} p^{2}\right)+\frac{\sigma_{m s}^{2}}{K_{1}^{2}}\left(4 m^{4}\left(1+\frac{m^{2}}{p^{2}}\right)\right) \\
& +\frac{\sigma_{t}^{2} c^{2}}{L^{2}}\left(4 p^{2}\left(m^{2}+p^{2}\right)\right),
\end{aligned}
$$

where $\sigma_{m^{2}}$ is the standard deviation of the $m^{2}$ distribution, $m$ denotes the physical mass of the particle and thus the square root of the centroid of the $m^{2}$ distribution, $\sigma_{\alpha}$ is the angular resolution of the drift chamber, $\sigma_{m s}$ is the multiple scattering term, $\sigma_{t}$ is the total timing resolution, and $K_{1}$ is the magnetic field integral constant. The magnetic field integral constant depends on the magnetic field configuration. The PHENIX magnet system for the central arms comprises two coils. The two coils can be run together, opposed, or with the inner coil off. During the $2007 \mathrm{Au}+\mathrm{Au}$ data taking, the coils were run opposed, while for the $2008 d+$ Au data taking, the coils were run together. Running the coils opposed produces zero magnetic field in the region between the beam-pipe and the inner coil of the magnet. This is needed for the analysis of the dielectron continuum using the hadron blind detector, which is the innermost detector during this operational period and can be seen (labeled as HBD) in Fig. 1. The PID parameters are presented in Table

TABLE II: Parameters for the PID function defined in Equation 2 .

\begin{tabular}{llrr}
\hline \hline Parameter & & $2007 \mathrm{Au}+\mathrm{Au}$ & $2008 d+\mathrm{Au}$ \\
\hline$\sigma_{\alpha}$ & $(\mathrm{mrad})$ & 0.896 & 1.050 \\
$\sigma_{m s}$ & $(\mathrm{mrad} \cdot \mathrm{GeV} / c)$ & 0.992 & 1.000 \\
$\sigma_{t}$ & $(\mathrm{ps})$ & 0.084 & 0.095 \\
$K_{1}$ & $(\mathrm{mrad} \cdot \mathrm{GeV} / \mathrm{c})$ & 75.0 & 104.0 \\
\hline \hline
\end{tabular}

To select candidate tracks of a particular particle species, the $m^{2}$ is required to be within two standard deviations of the mean for the selected particles species and outside two standard deviations of the mean for the other particle species. Below the regions where the PID bands intersect, the PID contamination is negligible. Above it, contamination is from one side of the tail of the distribution beyond 2 standard deviations. For a Gaussian distribution this is $2.25 \%$. Therefore the contamination can be estimated based on the ratio of the respective yields multiplied by this value. The $m^{2}$ distributions may have slightly nonGaussian tails and therefore the PID contamination may be slightly higher. In any event, we estimate the PID purity to be better than $90 \%$ for all particle species at all $p_{T}$, in all centrality classes, and in both collision systems. Figure 2 shows the $m^{2}$ vs the $p_{T}$ multiplied by the charge for the $2007 \mathrm{Au}+\mathrm{Au}$ data; the $2008 d+\mathrm{Au}$ data are very similar. The $2 \sigma$ PID bands are superimposed as solid black lines. The upper panel shows the entire $m^{2}$ distribution with all the track selection cuts applied but none of the PID cuts. The lower panel shows the same distribution but with the PID cuts also applied.

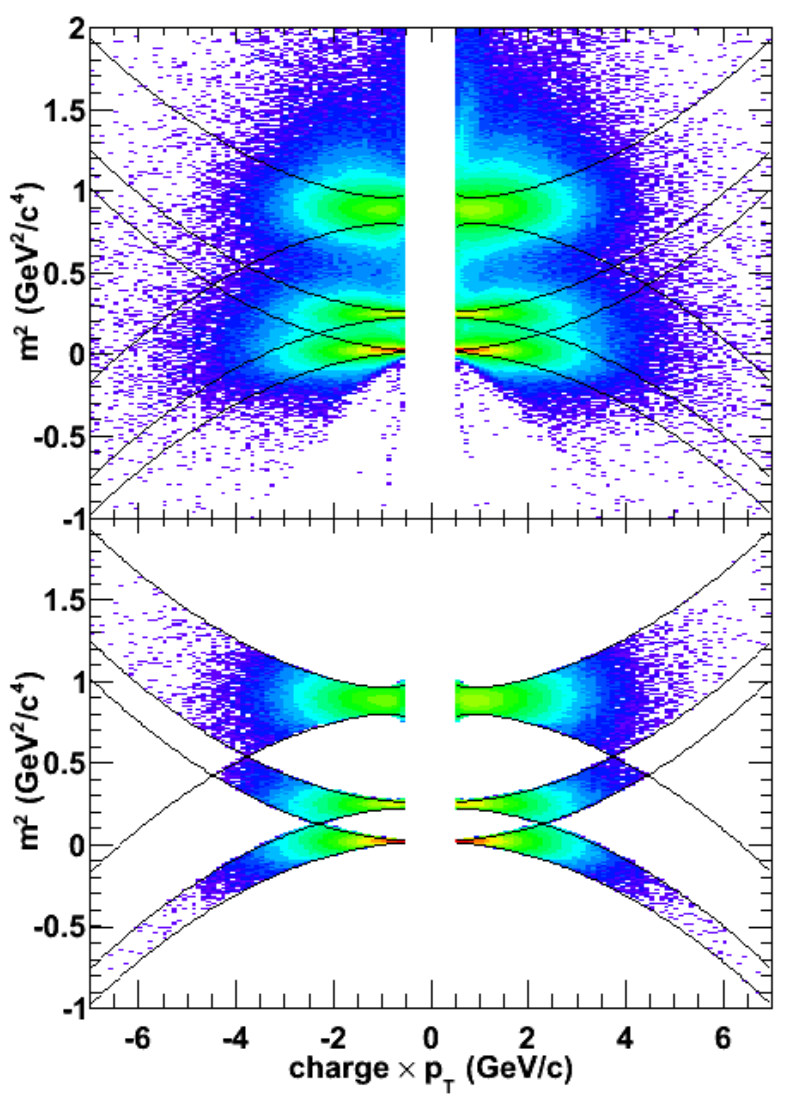

FIG. 2: (Color online) The particle identification method, $m^{2}$ vs charge $\times p_{T}$ for $2007 \mathrm{Au}+\mathrm{Au}$ data; the $2008 d+\mathrm{Au}$ data are very similar. The solid black lines indicate the two standard deviation PID bands used for the cuts. The upper panel shows the bands superimposed on the entire $\mathrm{m}^{2}$ distribution, the lower panel shows the distribution after the cuts have been applied.

\section{Corrections to the Raw Data}

In order to obtain the true invariant yield, the raw spectrum needs to be corrected for a variety of factors. Various types of simulations are performed to determine these corrections. To correct for geometrical acceptance, 
analysis cuts, particle interactions with detector materials, and in-flight decays (for pions and kaons), we use single particle Monte Carlo (MC) simulations. For these simulations, single particles are generated using a random generator, with flat distributions in rapidity, azimuth, and $p_{T}$. The random particles are then run through a GEANT simulation of PHENIX to determine the interactions of the single particles with the detector subsystems and support structures. Next, all the detector response information is fed through the usual PHENIX reconstruction software to produce simulated tracks. Finally, these simulated tracks are analyzed in the exact same way as tracks from the real data in order to determine the corrections. The total correction factor, $F_{C}\left(p_{T}\right)$, is given by the following relation:

$$
F_{C}\left(p_{T}\right)=\frac{d N_{\text {output }} / d p_{T}}{d N_{\text {input }} / d p_{T}}=\epsilon_{\text {acceptance }} \epsilon_{\text {efficiency }} \epsilon_{\text {cuts }}
$$

To correct for the detector occupancy effect, which is most significant in the TOFW, we run embedding simulations, where a track from single particle MC simulations is embedded into a real event, and the occupancy correction is determined from the relative efficiencies of reconstructing the single track in isolation and in the event. This correction is the largest in the most central $\mathrm{Au}+\mathrm{Au}$ collisions where the multiplicity is the highest and therefore the occupancy effect is the strongest. In the most peripheral $\mathrm{Au}+\mathrm{Au}$ collisions the multiplicity is low enough that there is essentially no effect. The same is true in $d+\mathrm{Au}$ collisions, where no correction is applied.

For the $d+$ Au system we apply a correction for the underlying event correlation that exists between produced particles measured in the central arms and particles at forward angles that satisfy the $\mathrm{BBC}$ interaction trigger [41]. This correlation produces both a trigger bias, in which events satisfying the trigger are biased towards higher multiplicities, and a bin shift, in which nominally peripheral events are shifted to higher centrality bins, thereby depleting the more peripheral bins. We correct for these effects using the Glauber Monte Carlo combined with central particle yields measured in $p+p$ collisions. Using this same framework, we also generate a correction factor to convert the minimum-bias sample (0-88\%) into one with zero-bias (0-100\%).

Table [II] shows the centrality dependent corrections for each collision species. The occupancy is represented as an efficiency, while the bias factor is represented as a multiplicative correction.

The proton and antiproton spectra are additionally corrected for the feed-down from weak decays of hyperons into protons. We use single particle MC simulations of the $\Lambda$ baryon and apply the analysis cuts used for the protons to determine the percentage of $\Lambda$ baryons that decay into protons that pass our proton selection cuts. This is used to determine the percentage of the total proton sample that likely comes from hyperon decays, which is called the feed-down fraction.
TABLE III: Summary of centrality dependent corrections.

\begin{tabular}{rr}
\hline \hline $\mathrm{Au}+\mathrm{Au}$ & Occupancy Dependent Efficiency \\
\hline $0-10 \%$ & 0.542 \\
$10-20 \%$ & 0.653 \\
$20-40 \%$ & 0.783 \\
$40-60 \%$ & 0.904 \\
$60-92 \%$ & 0.964 \\
\hline$d+\mathrm{Au}$ & Bias Factor Correction \\
\hline $0-20 \%$ & 0.94 \\
$20-40 \%$ & 1.00 \\
$40-60 \%$ & 1.03 \\
$60-88 \%$ & 1.03 \\
$0-100 \%$ & 0.89 \\
\hline \hline
\end{tabular}

The feed-down fraction is only dependent on the $\Lambda / p$ ratio and not explicitly on the $\Lambda$ spectrum itself. For the $\mathrm{Au}+\mathrm{Au}$ data we take the $\Lambda / p$ ratio to be 0.89 and the $\bar{\Lambda} / \bar{p}$ ratio to be 0.95 , as measured in $\mathrm{Au}+\mathrm{Au}$ collisions at $\sqrt{s_{N N}}=130 \mathrm{GeV}$ [42]. These are very similar to (and well within the systematic uncertainties of) the values of 0.91 for $\Lambda / p$ and 0.94 for $\bar{\Lambda} / \bar{p}$ obtained for $\mathrm{Au}+\mathrm{Au}$ collisions at $\sqrt{s_{N N}}=200 \mathrm{GeV}$ by examining the $d N / d y$ values for $\Lambda$ and $\bar{\Lambda}$ [43] and $p$ and $\bar{p}[10]$. We note however that the $\Lambda$ data reported in Ref. [42] are inclusive while the data $\Lambda$ reported in Ref. [43] are corrected for feeddown from other hyperons.

For the $d+\mathrm{Au}$ data, we take the $\Lambda / p$ ratio to be 0.85 and the $\bar{\Lambda} / \bar{p}$ ratio to be 0.99 . There are no published data on $\Lambda / p$ and $\bar{\Lambda} / \bar{p}$ ratios nor $\Lambda$ and $\bar{\Lambda}$ spectra or yields in $d+\mathrm{Au}$ collisions that could be used to estimate these ratios. Instead, we use the $(\Lambda+\bar{\Lambda}) /(p+\bar{p})$ ratio measured in $p+\bar{p}$ collisions [44], the $\bar{p} / p$ ratio measured in $d+\mathrm{Au}$ collisions [25], and an estimate of the $\bar{\Lambda} / \Lambda$ ratio in $d+\mathrm{Au}$ collisions based on measurements in $\mathrm{Au}+\mathrm{Au}$ [45] and $p+p$ collisions [46].

The spectral shape of the $\Lambda(\bar{\Lambda})$ is assumed to follow the $p(\bar{p})$ spectrum with $m_{T}$ scaling. We also take the ratio to be independent of centrality. In fact, a small centrality dependence can be seen when examining the integrated yields in Refs. [10] and [43], although the different centralities are consistent within the systematic uncertainties.

All measurements of $\Lambda$ implicitly include the $\Sigma^{0}$, which decays electromagnetically with $100 \%$ branching ratio to the $\Lambda$ and a photon. We do not correct for feeddown from the charged $\Sigma$ states, nor for the $\Xi$ and $\Omega$ multistrange baryon states. Because these corrections are smaller and have large uncertainties, they are included in the overall systematic uncertainty estimates. For the charged $\Sigma$ states we are concerned only with the $\Sigma^{+}$, which decays to a proton with a 0.5 branching ratio. The data from $200 \mathrm{GeV} p+\bar{p}$ collisions show a ra- 
tio $\left(\Sigma^{+}+\Sigma^{-}+\bar{\Sigma}^{+}+\bar{\Sigma}^{-}\right) /(\Lambda+\bar{\Lambda})$ of $0.50 \pm 0.18$ (syst) Ref. [44]. Assuming $\bar{\Sigma} / \Sigma \approx \bar{\Lambda} / \Lambda$ and $\Sigma^{-} / \Sigma^{+} \approx 1$, we can estimate $\Sigma^{+} / \Lambda$ to be 0.25 . The ratios for $\left(\Xi^{0}+\bar{\Xi}^{0}\right) /(\Lambda+\bar{\Lambda})$ and $\left(\Xi^{-}+\bar{\Xi}^{+}\right) /(\Lambda+\bar{\Lambda})$ in $p+\bar{p}$ collisions both have a value of 0.065 with very large $(\approx 100 \%)$ systematic uncertainties. Assuming $\Xi / \Xi \approx \bar{\Lambda} / \Lambda$ one can estimate the $\left(\Xi^{0}+\Xi^{-}\right) / \Lambda$ ratio to be roughly 0.13 , which is consistent with the total feed-down correction of $15 \%$ reported for $\Lambda$ in Ref. [43]. The latter also includes feed-down from $\Omega$, though the contribution is small.

The systematic uncertainty on the feed-down fraction is estimated to be $25 \%$, which is due primarily to the uncertainty in the $\Lambda / p$ ratio. We also note that, in the $p_{T}$ region of interest to this paper, $2 \mathrm{GeV} / c<p_{T}<5 \mathrm{GeV} / c$, the feed-down fraction is of order $10 \%$, so a $25 \%$ change in the $\Lambda / p$ ratio produces only a roughly $2.5 \%$ change in the proton spectrum.

Figure 3 shows the feed-down fraction as a function of $p_{T}$ for protons as solid black lines and antiprotons as dotted red lines for the $2007 \mathrm{Au}+\mathrm{Au}$ data (left panel) and $2008 d+\mathrm{Au}$ data (right panel). As mentioned above, the magnetic field configuration is different for 2007 and 2008 , which is the reason for the different feed-down fractions between the two data sets.

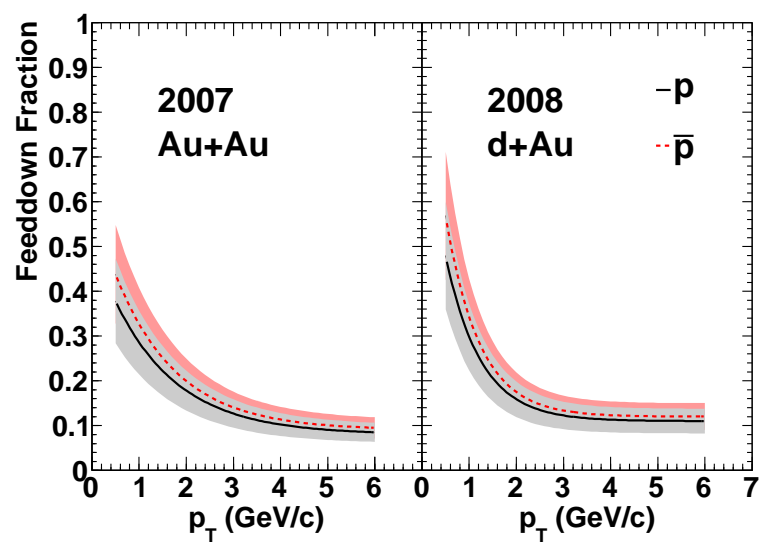

FIG. 3: (Color online) Feed-down fraction as a function of $p_{T}$ for protons (sold black lines) and antiprotons (dotted red lines) for the $2007 \mathrm{Au}+\mathrm{Au}$ data (left panel) and the 2008 $d+\mathrm{Au}$ data (right panel). The shaded bands indicate the systematic uncertainty of $25 \%$.

\section{Estimation of Systematic Uncertainties}

The different types of systematic uncertainty are categorized as follows: type A, point-to-point uncorrelated in $p_{T}$, where the points can move up or down independently of each other; type B, point-to-point correlated in $p_{T}$, where the points can move together changing the shape of the curve; and type $\mathrm{C}$, an overall normalization uncertainty in which all points move up or down by the same factor. The corrections for analysis cuts, including acceptance, track selection, and PID, are predominantly type B. The normalization corrections for effects such as detector occupancy and efficiency are type C. The uncertainties are assessed for each cut by redoing the analysis with the cut varied and then determining the difference. The cuts are varied in exactly the same way for the analysis of both the experimental and the simulated data. This process is repeated for all the analysis cuts and the differences are summed in quadrature to determine the final uncertainty. This is done for both the spectra and for the ratios. The uncertainties are examined in each centrality class for both $\mathrm{Au}+\mathrm{Au}$ and $d+\mathrm{Au}$ collisions. In $\mathrm{Au}+\mathrm{Au}$ collisions, they are found to be quite similar in all centrality classes. In $d+\mathrm{Au}$ collisions they are found to be negligibly different for all centrality classes. When taking ratios of the various particles, all of the type $\mathrm{C}$ uncertainties and some of the type B uncertainties cancel. For antiparticle to particle ratios, the uncertainty from acceptance does not cancel at all, and the uncertainty from track selection and PID mostly cancel. For other particle ratios, $K / \pi$ and $p / \pi$, the uncertainty from acceptance and track selection mostly cancel, and the uncertainty from PID does not cancel at all. In this analysis, we find the remaining systematic uncertainty on each of the particle ratios is roughly $5 \%$ for all $p_{T}$. For the nuclear modification factor $R_{C P}$, which compares two different centrality bins of the same particle species in the same collision system, all the type B systematic uncertainties cancel almost completely. There is an uncertainty of about $2 \%$ based on small variations of the track matching and PID distributions as a function of centrality. The type $\mathrm{C}$ uncertainties are completely uncorrelated and added in quadrature. For the nuclear modification factors $R_{\mathrm{AA}}$ and $R_{d A}$, the $p+p$ reference data were collected during a different operational period and using different detector subsystems, therefore none of the systematic uncertainties cancel. A summary of the type B systematic uncertainties for the spectra is given in Table IV

TABLE IV: Summary of systematic uncertainties from acceptance, track selection, and PID of invariant yield of each particle species.

\begin{tabular}{lrrrrrr}
\hline \hline & $\pi^{+}$ & $\pi^{-}$ & $K^{+}$ & $K^{-}$ & $p$ & $\bar{p}$ \\
\hline $2007 \mathrm{Au}+\mathrm{Au}$ & & & & & & \\
$p_{T}<3 \mathrm{GeV} / c$ & $9 \%$ & $9 \%$ & $11 \%$ & $11 \%$ & $10 \%$ & $10 \%$ \\
$p_{T}>3 \mathrm{GeV} / c$ & $10 \%$ & $10 \%$ & $11 \%$ & $11 \%$ & $11 \%$ & $11 \%$ \\
$p_{T}>5 \mathrm{GeV} / c$ & $14 \%$ & $14 \%$ & - & - & $14 \%$ & $14 \%$ \\
\hline $2008 d+\mathrm{Au}$ & & & & & & \\
$p_{T}<3 \mathrm{GeV} / c$ & $8 \%$ & $8 \%$ & $13 \%$ & $13 \%$ & $9 \%$ & $9 \%$ \\
$p_{T}>3 \mathrm{GeV} / c$ & $9 \%$ & $9 \%$ & $13 \%$ & $13 \%$ & $11 \%$ & $11 \%$ \\
\hline \hline
\end{tabular}

The type $\mathrm{C}$ uncertainties are from the centrality dependent corrections for the spectra and from the uncertainty on the Glauber model calculations for the nuclear modification factors. The uncertainty on the occupancy correc- 
tion for $\mathrm{Au}+\mathrm{Au}$ collisions is roughly $10 \%$, and therefore the uncertainty on the yield varies from $5 \%$ for the most central to less than $1 \%$ for the most peripheral. The uncertainties for the Glauber values for $N_{\text {coll }}$ are much larger and therefore dominate the uncertainty in the nuclear modification factors. The uncertainty for the bias factors for $d+\mathrm{Au}$ collisions varies from about $1 \%$ for the most central bin to about $5 \%$ for the most peripheral bin. The bias factors are determined in the same Glauber model analysis as the $N_{\text {coll }}$ and $N_{\text {part }}$ values, and therefore the uncertainties are correlated. The uncertainty on the ratio of bias factors and $N_{\text {coll }}$ values used to determine the nuclear modification factors varies from about $3 \%$ for the most central to about $8 \%$ for the most peripheral.

\section{RESULTS AND DISCUSSION}

\section{A. Invariant Yield as a Function of Transverse Momentum $p_{T}$}

The main result of this study is the measurement of the invariant yield of pions, kaons, and protons as a function of $p_{T}$ in different centrality classes. The centrality classes studied in the $\mathrm{Au}+\mathrm{Au}$ measurement are 0$10 \%$ (the most central), 10-20\%, 20-40\%, 40-60\%, and 60-92\% (the most peripheral). For the $d+\mathrm{Au}$ measurement, the centrality classes are 0-20\% (the most central), 20-40\%, 40-60\%, 60-88\% (the most peripheral), and 0$100 \%$. From these quantities, all other observables are derived, such as particle ratios and nuclear modification factors. Figure 4 shows the invariant yields of positive pions, positive kaons, and protons (upper left, middle, and right, respectively), and negative pions, negative kaons, and antiprotons (lower left, middle, and right, respectively). The yields are scaled by arbitrary factors indicated in the legend for the sake of clarity and to keep the collision species grouped together.

\section{B. Particle Ratios as a Function of Transverse Momentum}

One of the simpler classes of derived quantities is the antiparticle to particle ratio. In the present analysis those ratios are $\pi^{-} / \pi^{+}, K^{-} / K^{+}$, and $\bar{p} / p$, which are plotted as a function of $p_{T}$ in the upper, middle, and lower panels of Fig. 5, respectively. In each panel, the $\mathrm{Au}+\mathrm{Au}$ data are on the left and the $d+\mathrm{Au}$ data are on the right. Drawn as a visual aid are dashed black lines with value 1.0 for the pions, 0.93 for the kaons, and 0.73 for the protons; the values for kaons and protons are chosen from the reported $p_{T}$ integrated values from Ref. [10]. Shown as a reference are data from $p+p$ collisions from Ref. [47] for $p_{T}<3 \mathrm{GeV} / c$ and from Ref. [48] for $p_{T}>3 \mathrm{GeV} / c$. Remarkably, all the ratios are essentially independent of both $p_{T}$ and centrality. Based on simple arguments about isospin conservation and the basics of the parton distribution functions and fragmentation functions, one would expect each of the antiparticle to particle ratios to vary as a function of $p_{T}$ as discussed in Ref. [49]. Indeed, these ratios have a significant $p_{T}$ dependence in $p+p$ collisions at midrapidity, and the agreement with theory depends significantly on the fragmentation functions used [48]. However, the $p_{T}$ range needed to observe the decrease in these ratios in $p+p$ collisions is quite large. As seen in Fig. [5 the $p_{T}$ dependence of the ratios in $p+p$ is small and may be consistent with the ratios in $d+\mathrm{Au}$ and $\mathrm{Au}+\mathrm{Au}$.

Figures 6 and 7 show the kaon to pion ratios as a function of $p_{T}\left(K^{+} / \pi^{+}\right.$on the top, $K^{-} / \pi^{-}$on the bottom) in $\mathrm{Au}+\mathrm{Au}$ and $d+\mathrm{Au}$ collisions, respectively. The ratios in $\mathrm{Au}+\mathrm{Au}$ collisions show a significant increase with increasing $p_{T}$ and a small increase as the collisions become more central. The enhancement of the integrated $K / \pi$ ratio in more central collisions is attributed to strangeness equilibration in various thermal models [50, 51], which is reflected in the differential ratio. However, the differential ratio may include additional information about the differences in the fragmentation functions and/or the phase space distribution functions used in the recombination models. As discussed in a previous PHENIX publication [52], the strangeness enhancement present in the hot and dense nuclear medium has an effect on certain recombination models [53]. These recombination models involve the recombination of partons in dissimilar momentum space, meaning that a shower parton from a jet can recombine with a thermal parton in the medium. The thermal component of thermal+shower recombination is more dominant at higher $p_{T}$ for strange hadrons (like kaons) than it is for nonstrange hadrons (like pions), leading to an enhancement of the ratio that increases with $p_{T}$. This increasing enhancement manifests as the ratio rising more quickly in $\mathrm{Au}+\mathrm{Au}$ collisions compared with $p+p$ collisions, which is seen in Figs. 6 and 7. At sufficiently high $p_{T}$ where the shower component begins to dominate for both strange and nonstrange particles, this ratio is expected to turn over and begin to decrease. However, this turnover point, if it exists, is beyond the $p_{T}$ reach available for kaons in this study.

The $K / \pi$ ratios in $d+$ Au collisions are essentially identical for all centrality classes, which may indicate that the mechanism for strangeness production in $d+$ Au collisions is the same for all centrality classes. However, we also note that the various $d+$ Au centrality classes span a relatively small range of $N_{\text {part }}$. Therefore, if the strangeness enhancement is only weakly dependent on $N_{\text {part }}$, the variation of $N_{\text {part }}$ in the $d+\mathrm{Au}$ centrality classes may not be large enough for an effect to be observed.

Figures 8 and 9 show the proton to pion ratios as a function of $p_{T}\left(p / \pi^{+}\right.$on the top, $\bar{p} / \pi^{-}$on the bottom) in $\mathrm{Au}+\mathrm{Au}$ and $d+\mathrm{Au}$ collisions, respectively. Note that for Fig. 9 the vertical scale is different. The ratios in central $\mathrm{Au}+\mathrm{Au}$ collisions show a strong enhancement over the values in $p+p$ collisions. This is conjectured to 

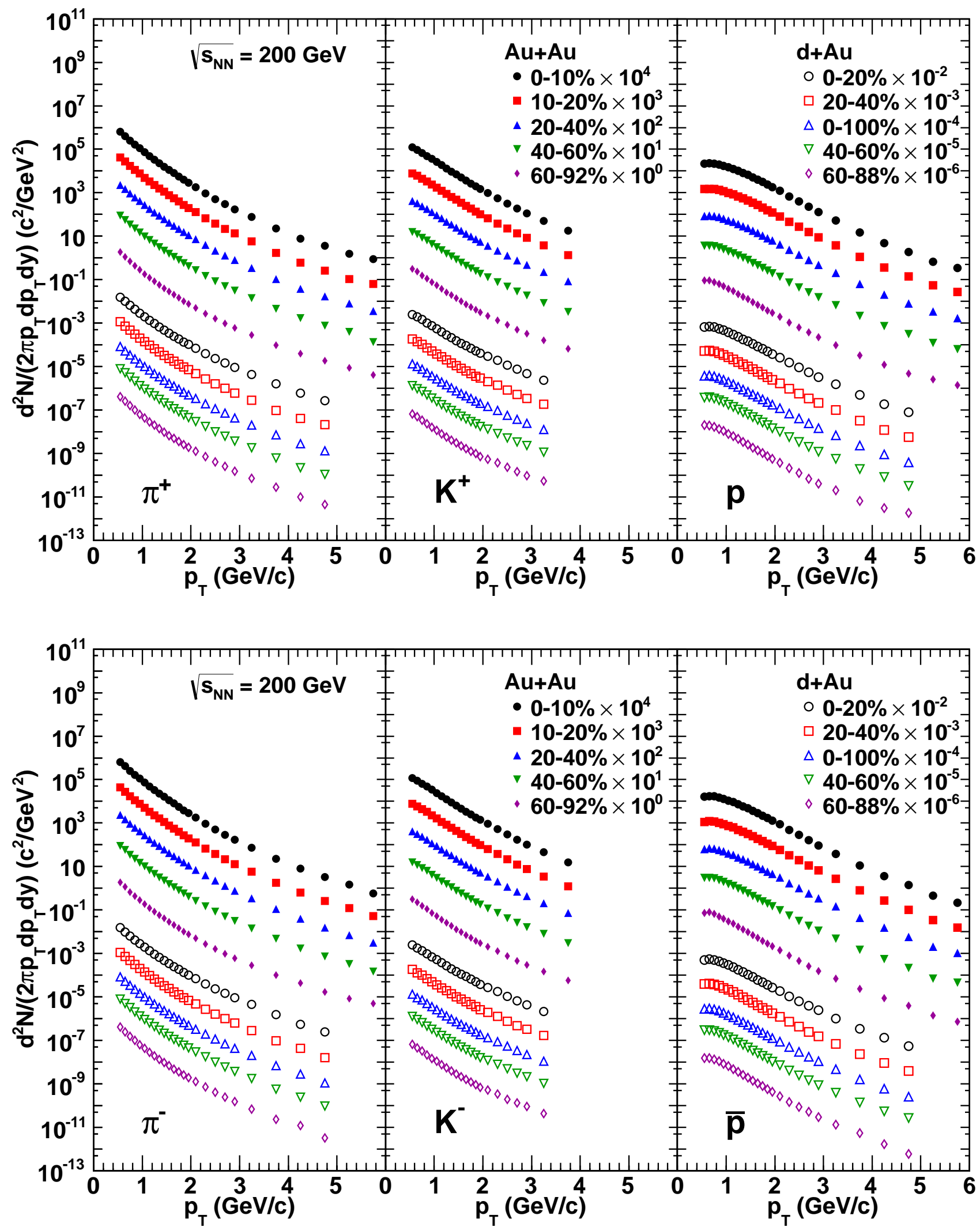

FIG. 4: (Color online) Invariant yield of $\pi^{ \pm}, K^{ \pm}$, and $p$ and $\bar{p}$ as a function of $p_{T}$ in Au+Au and $d+$ Au collisions. The yields are scaled by the arbitrary factors indicated in the legend, keeping collisions species grouped together. 

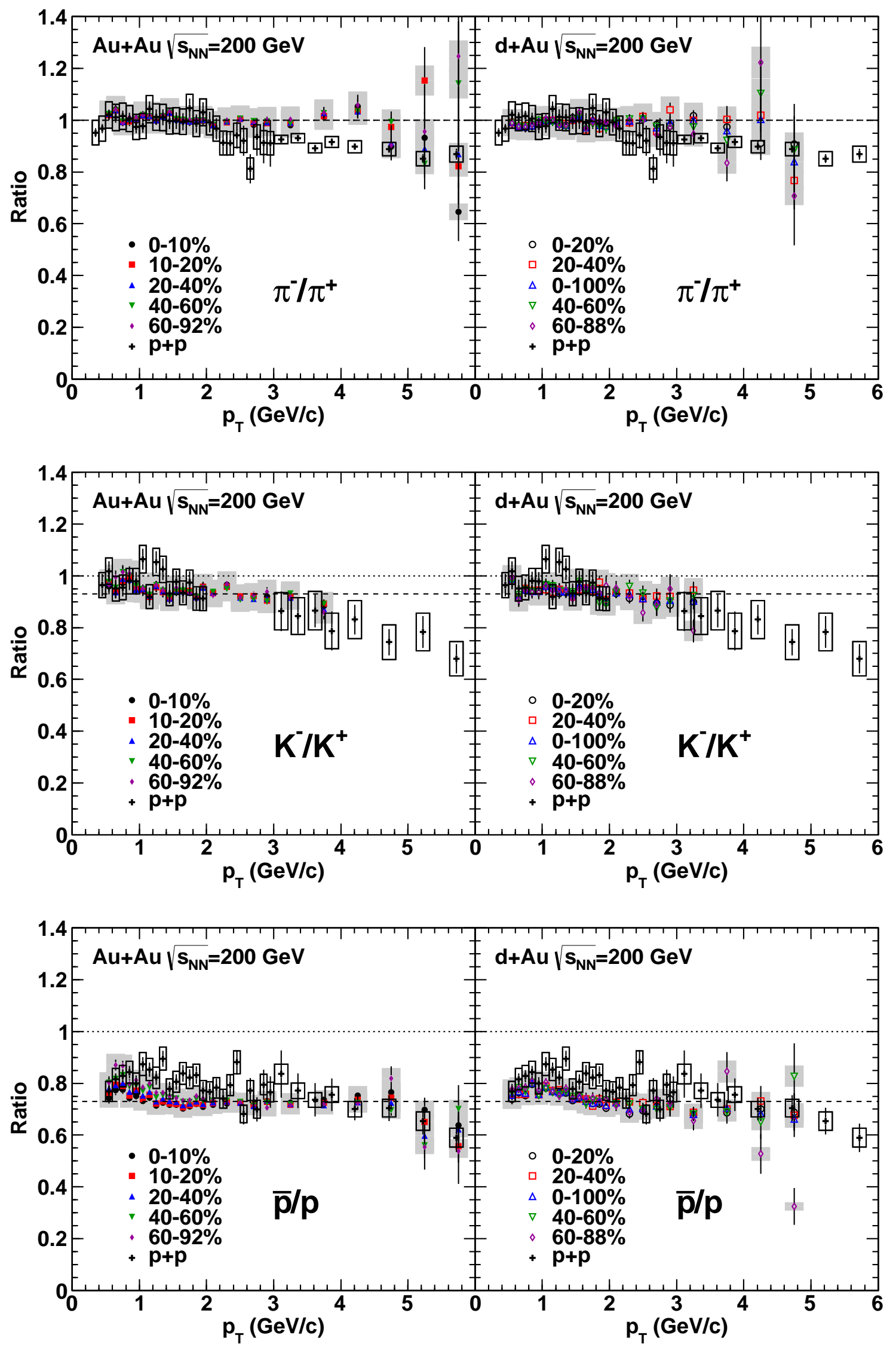

FIG. 5: (Color online) Ratio of invariant yield of $\pi^{-} / \pi^{+}$(top), $K^{-} / K^{+}$(middle), and $\bar{p} / p$ (bottom) as a function of $p_{T}$ in $\mathrm{Au}+\mathrm{Au}$ (left panels) and $d+\mathrm{Au}$ collisions (right panels) in each centrality bin. Dashed lines are drawn as a visual aid with values of 1.0 for $\pi^{-} / \pi^{+}, 0.93$ for $K^{-} / K^{+}$, and 0.73 for $\bar{p} / p$. These values are taken from Ref. [10]. Shown as a reference are data from $p+p$ collisions from Ref. [47] for $p_{T}<3 \mathrm{GeV} / c$ and from Ref. [48] for $p_{T}>3 \mathrm{GeV} / c$. 


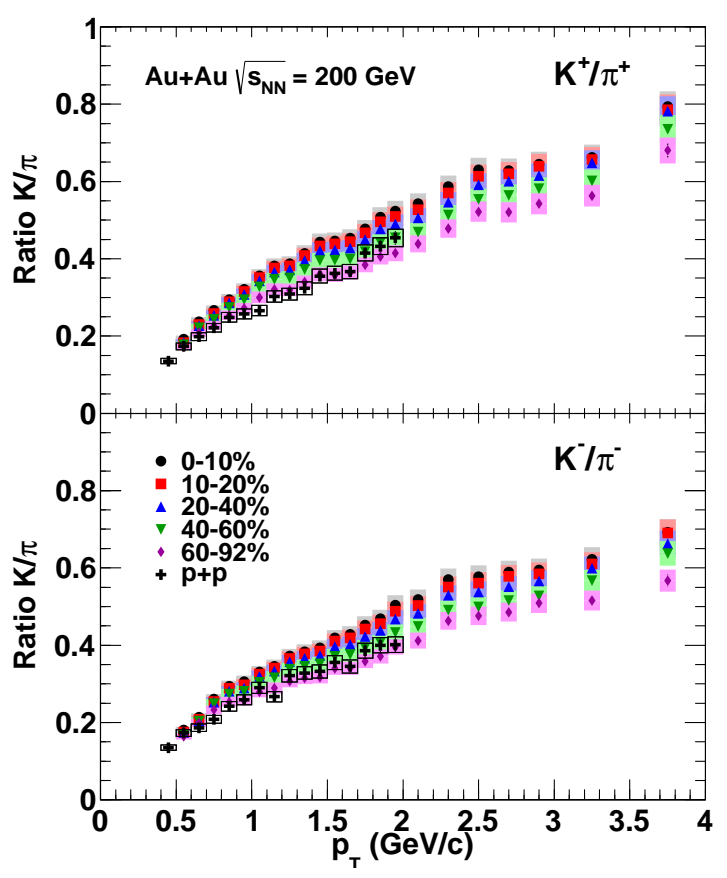

FIG. 6: (Color online) Ratio of invariant yield of positive kaons to positive pions (upper panel) and negative kaons to negative pions (lower panel) as a function of $p_{T}$ in $\mathrm{Au}+\mathrm{Au}$ collisions in the centrality bins marked in the legend. Data for $p+p$ collisions [47] are shown as a reference.

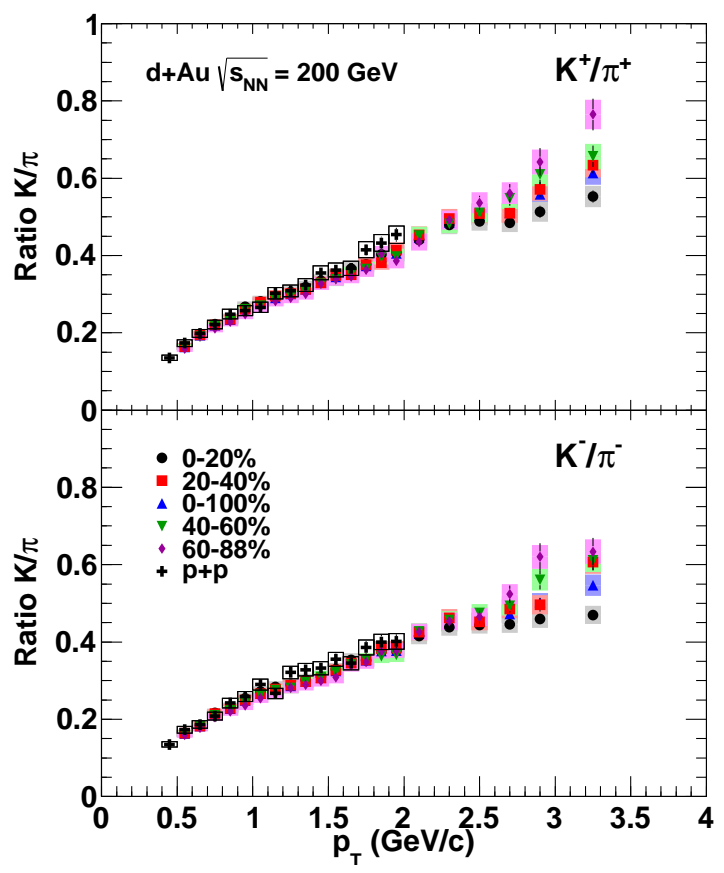

FIG. 7: (Color online) Ratio of invariant yield of positive kaons to positive pions (upper panel) and negative kaons to negative pions (lower panel) as a function of $p_{T}$ in $d+\mathrm{Au}$ collisions in the centrality bins marked in the legend. Data for $p+p$ collisions [47] are shown as a reference.

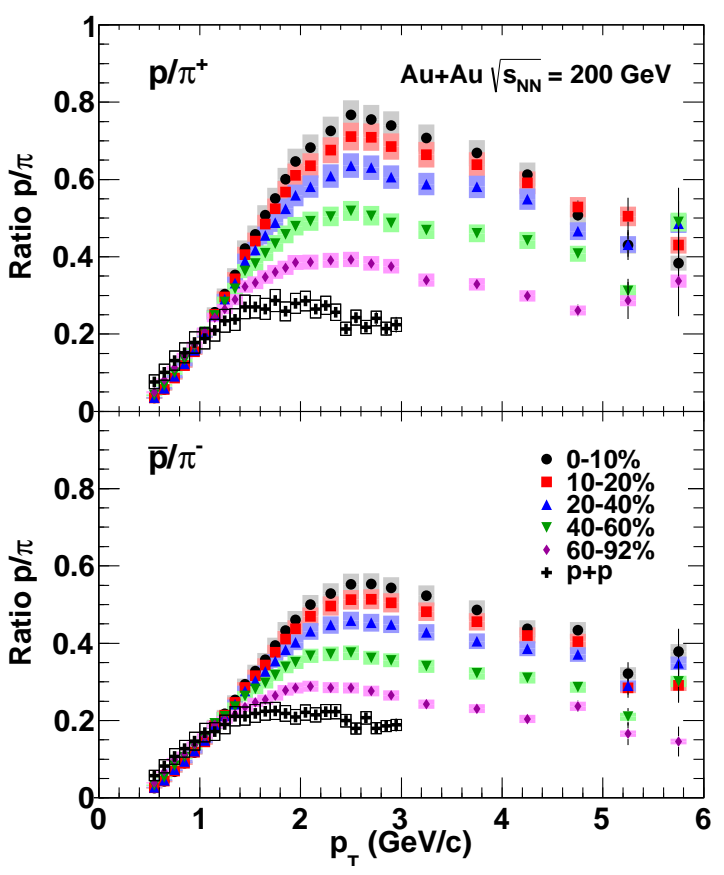

FIG. 8: (Color online) Ratio of invariant yield of protons to positive pions (upper panel) and antiprotons to negative pions (lower panel) as a function of $p_{T}$ in $\mathrm{Au}+\mathrm{Au}$ collisions in the centrality bins marked in the legend. Data for $p+p$ collisions [47] are shown as a reference.

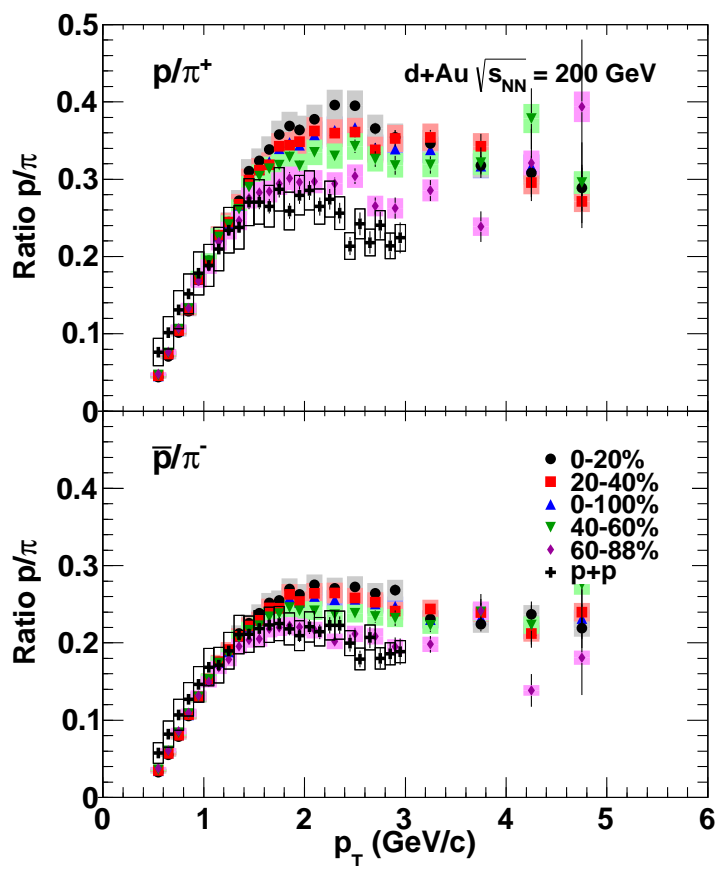

FIG. 9: (Color online) Ratio of invariant yield of protons to positive pions (upper panel) and antiprotons to negative pions (lower panel) as a function of $p_{T}$ in $d+\mathrm{Au}$ collisions in the centrality bins marked in the legend. Data for $p+p$ collisions [47] are shown as a reference. 


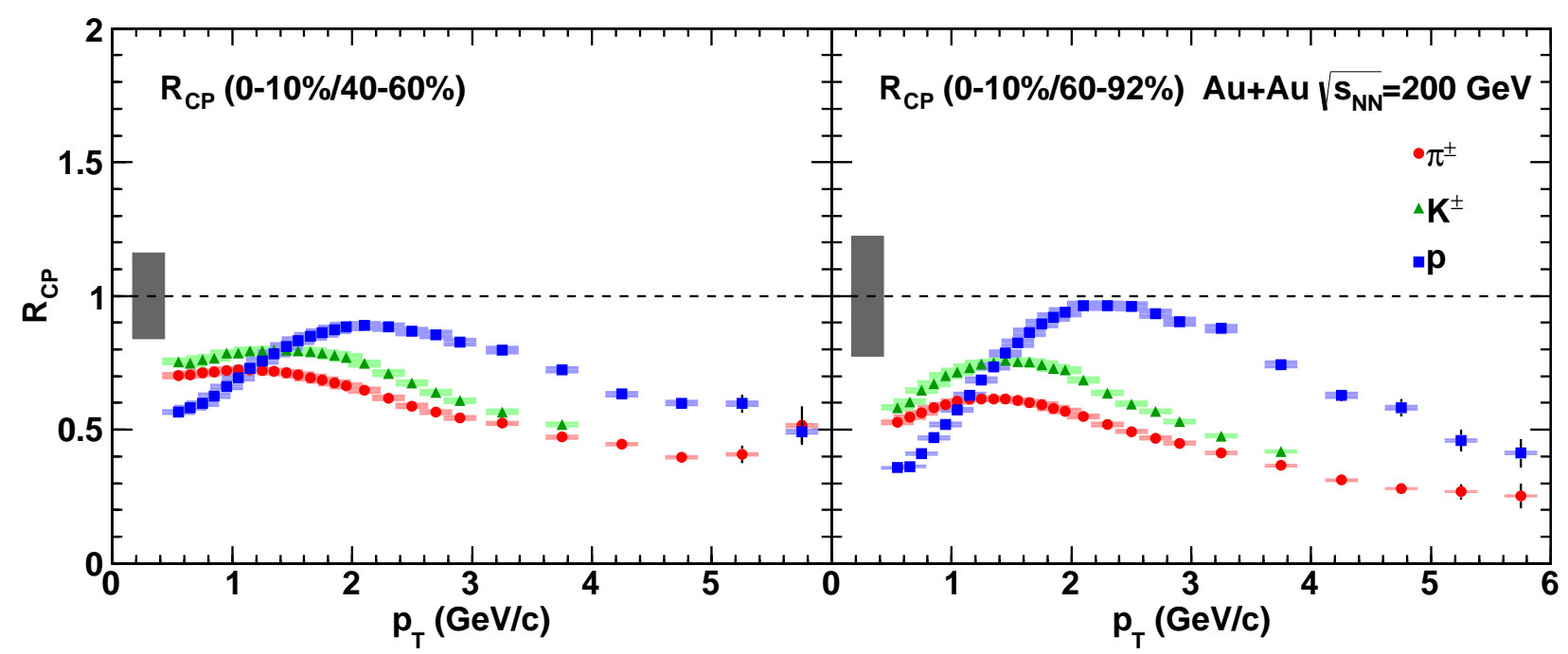

FIG. 10: (Color online) $R_{C P}$ for 0-10\%/40-60\% (left panel) and 0-10\%/60-92\% (right panel) as a function of $p_{T}$ for charge averaged pions, kaons, and protons. A dashed black line is drawn at unity as a visual aid, indicating nonmodification. The shaded gray boxes indicate the associated uncertainty on $N_{\text {coll }}$ from the Glauber model calculations.

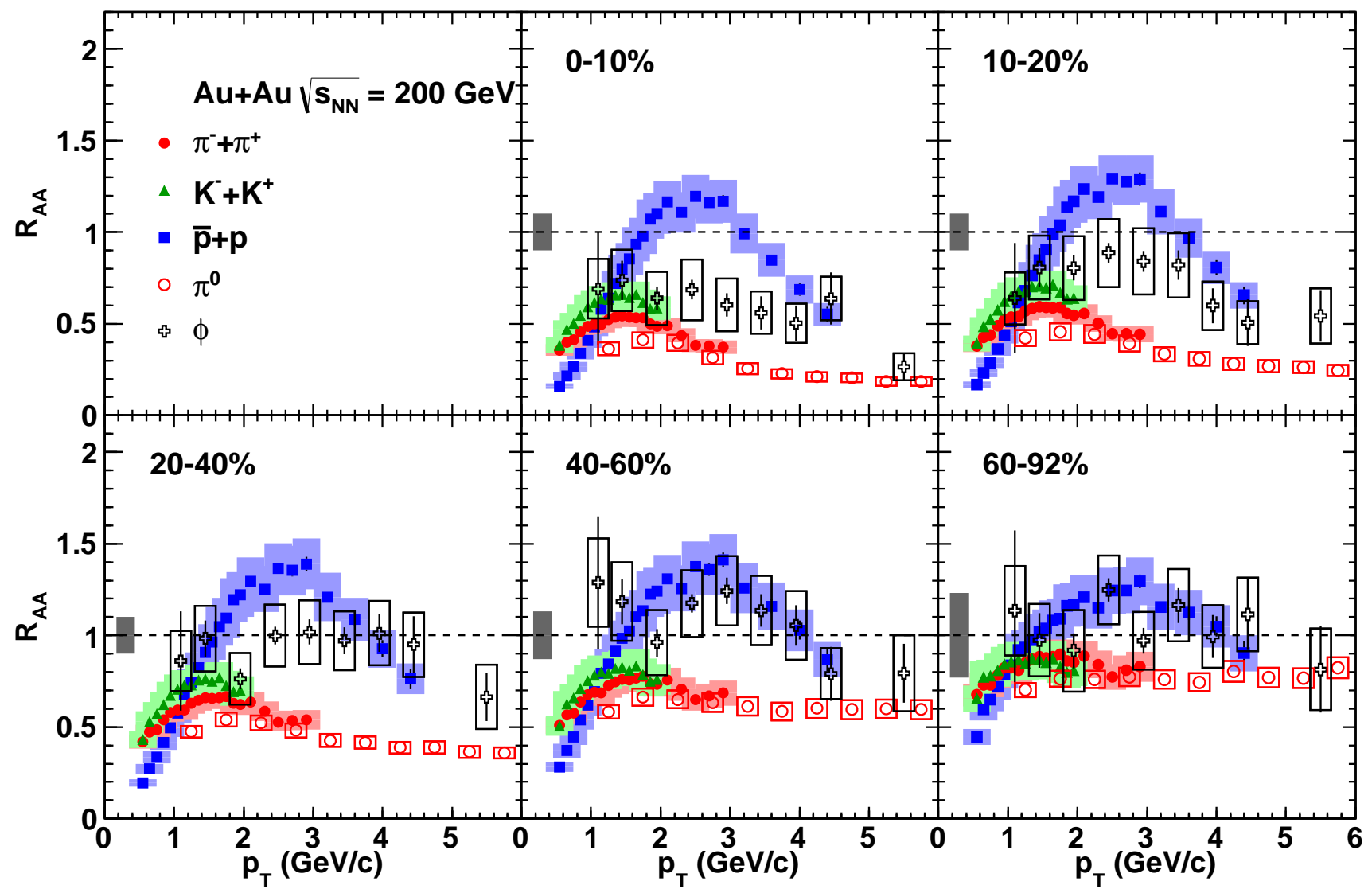

FIG. 11: (Color online) Nuclear modification factor $R_{\mathrm{AA}}$ as a function of $p_{T}$ in different centrality classes of charge averaged pions, kaons, and protons, $\pi^{0}$ [54], and $\phi$ [52]. A dashed black line is drawn at unity as a visual aid, indicating nonmodification. The shaded gray boxes indicate the associated uncertainty on $N_{\text {coll }}$ from the Glauber model calculations. 
be attributed to the parton recombination mechanism of hadronization, which gives rise to a significant enhancement of baryon yields relative to meson yields in heavy ion collisions $16,18,20]$. The $p / \pi$ ratios in the other centralities in $\mathrm{Au}+\mathrm{Au}$ collisions show a clear and consistent trend with decreasing enhancement as the collisions become more peripheral. In $d+\mathrm{Au}$ collisions there is a similar trend. The $p / \pi$ ratio in the most central $d+\mathrm{Au}$ collisions appears consistent with the ratio in the most peripheral $\mathrm{Au}+\mathrm{Au}$ collisions. Additionally, the $p / \pi$ ratio is enhanced over $p+p$ collisions for each centrality class in $d+\mathrm{Au}$ collisions except for the most peripheral.

\section{Nuclear Modification Factors as a Function of Transverse Momentum}

To measure the modification of the spectrum of produced particles in heavy ion collisions relative to the spectrum in $p+p$ collisions, nuclear modification factors are employed. The nuclear modification factor $R_{\mathrm{AA}}$ is defined as the yield in $\mathrm{Au}+\mathrm{Au}$ collisions divided by the yield in $p+p$ collisions, normalized by the number of binary nucleon+nucleon collisions $N_{\text {coll }}$ as determined from the Glauber model. The nuclear modification factor $R_{C P}$ is defined as the yield in central $\mathrm{Au}+\mathrm{Au}$ collisions divided by the yield in peripheral $\mathrm{Au}+\mathrm{Au}$ collisions, normalized to the respective numbers of binary nucleon+nucleon collisions. These can be expressed mathematically as:

$$
\begin{gathered}
R_{\mathrm{AA}}=\frac{\left(d N / d p_{T}\right)^{\mathrm{Au}+\mathrm{Au}}}{N_{\mathrm{coll}}^{\mathrm{Au}+\mathrm{Au}}\left(d N / d p_{T}\right)^{p+p}}, \\
R_{C P}=\frac{\left(d N / d p_{T}\right)^{\text {central }}}{\left(d N / d p_{T}\right)^{\text {peripheral }}} \frac{N_{\text {coll }}^{\text {peripheral }}}{N_{\text {coll }}^{\text {central }}} .
\end{gathered}
$$

Figure 10 shows $R_{C P}$ for $0-10 \% / 40-60 \%$ (left panel) and $0-10 \% / 60-92 \%$ (right panel) as a function of $p_{T}$ for charge averaged pions, kaons, and protons. Both pions and kaons exhibit a suppression pattern at all values of $p_{T}$. The kaons exhibit less suppression than the pions, indicating the additional role of strangeness enhancement in the particle production mechanism. The observed enhancement of kaons relative to pions appears to be lower for the $0-10 \% / 40-60 \%$ as compared to the 0 $10 \% / 60-92 \%$, suggesting a centrality dependence of the strangeness enhancement, as seen in the $K / \pi$ ratios discussed above. The protons on the other hand exhibit quite different behavior, rising to a value very close to unity, indicating nonsuppression, around $2-3 \mathrm{GeV} / c$ in $p_{T}$. At higher values of $p_{T}$ the proton $R_{C P}$ falls off slowly, beginning to approach the pion $R_{C P}$ at the highest values of $p_{T}$ available. The proton $R_{C P}$ shown here is consistent within the systematic uncertainties with the proton $R_{C P}$ reported by STAR [56].

Figure 11 shows $R_{\mathrm{AA}}$ as a function of $p_{T}$ in different centrality classes for charge averaged pions, kaons, and protons, as well for $\pi^{0}$ [54] and $\phi[52]$. We use previously published PHENIX data on identified hadrons in $p+p$ collisions [47] to evaluate the $R_{\mathrm{AA}}$. The $R_{\mathrm{AA}}$ data are limited in $p_{T}$ reach by the $p+p$ data. As with the $R_{C P}$, the pions and kaons exhibit a suppression pattern in the $R_{\mathrm{AA}}$. Additionally, a significant and monotonic centrality dependence is observed, with the suppression decreasing as the collisions become more peripheral. This is consistent with what is seen for neutral pions [54, 57]. The proton $R_{\mathrm{AA}}$ shows no suppression in the intermediate $p_{T}$ region and in fact reaches a maximum value above unity between $2-3 \mathrm{GeV} / c$. For $p_{T}>3 \mathrm{GeV} / c$, the proton $R_{\mathrm{AA}}$ values decrease and a suppression pattern emerges. The proton $R_{C P}$ decreases more slowly than the central proton $R_{\mathrm{AA}}$, which is simply because of the still considerable modification in the peripheral bins. The trend appears to be that the proton $R_{C P}$ and $R_{\mathrm{AA}}$ decrease steadily while the pion $R_{C P}$ and $R_{\mathrm{AA}}$ hold steady, suggesting that these values for pions and protons may eventually merge. The proton $R_{\mathrm{AA}}$ for the $0-10 \%$ centrality bin shown here exhibits reasonable qualitative agreement with the $K+p R_{\mathrm{AA}}$ for $0-12 \%$ centrality reported by STAR [8].

While the centrality dependence of the $R_{\mathrm{AA}}$ for the pions and kaons is strong, it is quite weak for the protons and the different centralities are consistent within the systematic uncertainties. This is consistent with the strong centrality dependence in the $p / \pi$ ratios discussed above. The $\phi$ meson $R_{\mathrm{AA}}$ values are close to the values for kaons, and significantly lower than the values for the protons, even though the $\phi$ is much heavier than the kaon and it has roughly the same mass as the proton. This strongly suggests a baryon vs. meson dynamic, as opposed to a simple mass dependence, as would be the case for radial flow developed during the hadronic phase.

The nuclear modification factor for $d+\mathrm{Au}$ collisions, $R_{d A}$, is defined in a similar way as $R_{\mathrm{AA}}$ by

$$
R_{d A}=\frac{\left(d N / d p_{T}\right)^{d+\mathrm{Au}+\mathrm{Au}}}{N_{\mathrm{coll}}^{d+\mathrm{Au}}\left(d N / d p_{T}\right)^{p+p}}
$$

Figure 12 shows $R_{d A}$ as a function of $p_{T}$ in different centrality classes for charged averaged particles. As with the $R_{\mathrm{AA}}$, we use previously published PHENIX data on identified hadrons in $p+p$ collisions [47] to evaluate the $R_{d A}$. The $p+p$ data limit the $p_{T}$ reach of the $R_{d A}$. The charged pion exhibits a small modification above $p_{T}$ of $1.0 \mathrm{GeV} / c$ and is consistent with nonmodification within the systematic uncertainties. This is consistent with previous measurements of neutral pions [55, 58]. The charged kaon agrees with the charged pion within the systematic uncertainties. The $\phi$ meson exhibits no apparent modification.

On the other hand, the protons show a very large and strongly centrality dependent Cronin enhancement, reaching a factor of 2 in the most central collisions at intermediate $p_{T}$. Even in the $40-60 \%$ centrality class the enhancement is a factor of 1.5. For the most periph- 


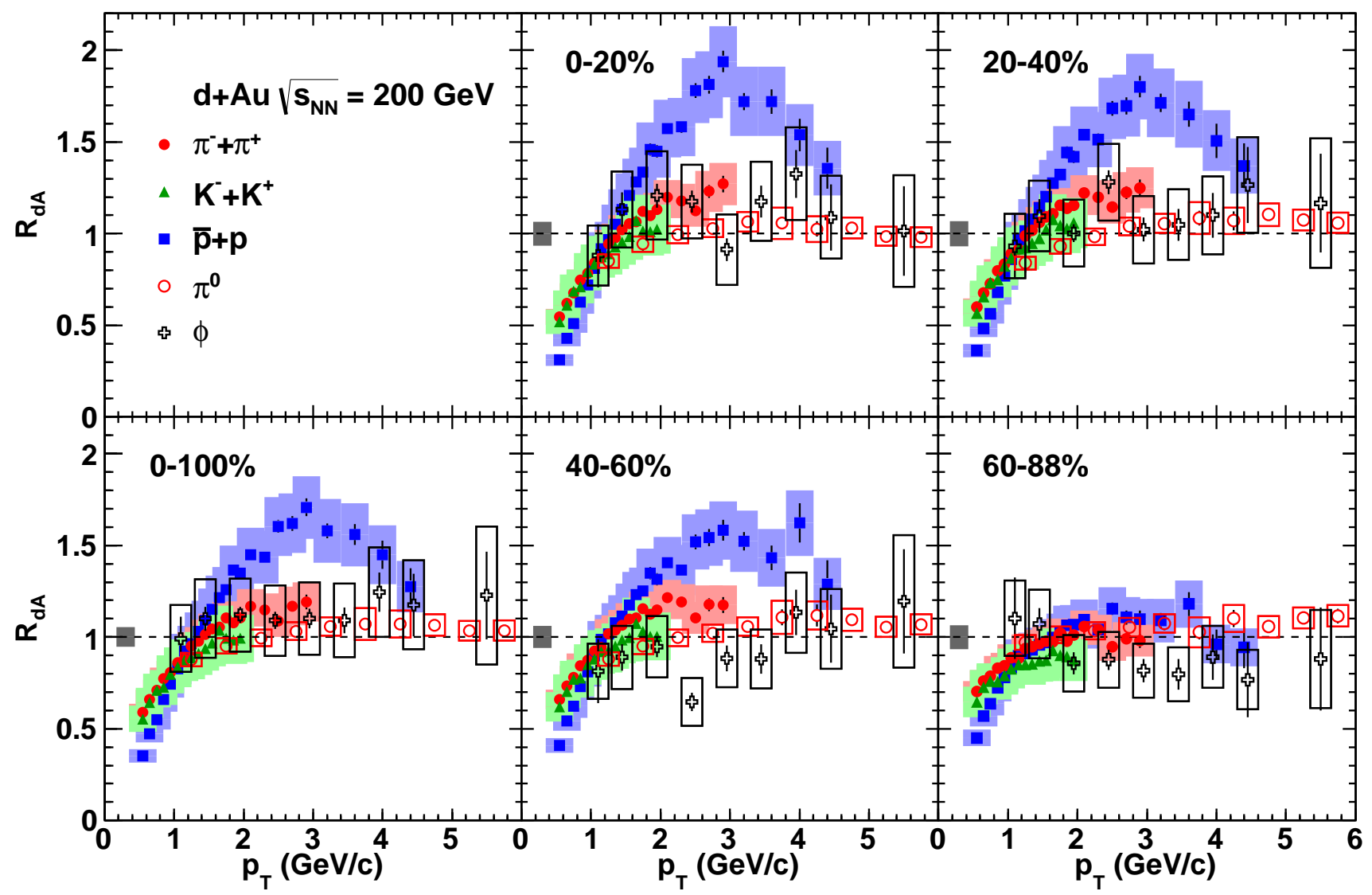

FIG. 12: (Color online) Nuclear modification factor $R_{d A}$ as a function of $p_{T}$ in different centrality classes of charge averaged pions, kaons, and protons, $\pi^{0}$ [55], and $\phi[52]$. A dashed black line is drawn at unity as a visual aid, indicating nonmodification. The shaded gray boxes indicate the associated uncertainty on $N_{\text {coll }}$ from the Glauber model calculations.

eral bin the enhancement is much smaller, at a factor of about 1.1-1.2, and is close to unmodified, similar to the other particle species. This strong centrality dependence

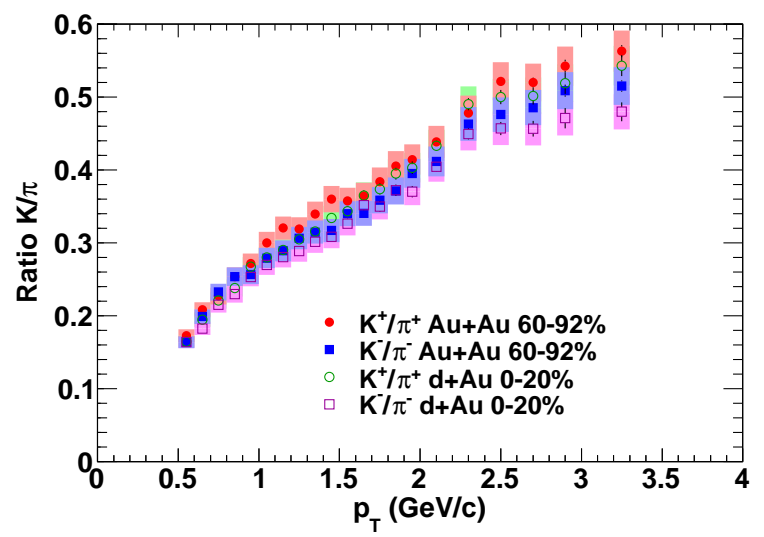

FIG. 13: (Color online) Ratio of $K^{+} / \pi^{+}$and $K^{-} / \pi^{-}$as a function of $p_{T}$ in peripheral $\mathrm{Au}+\mathrm{Au}$ and central $d+\mathrm{Au}$ collisions plotted together. of the proton $R_{d A}$ is in fact very similar to the significant centrality dependence of the $p / \pi$ ratio, and these two observables are likely driven by the same mechanism. Also

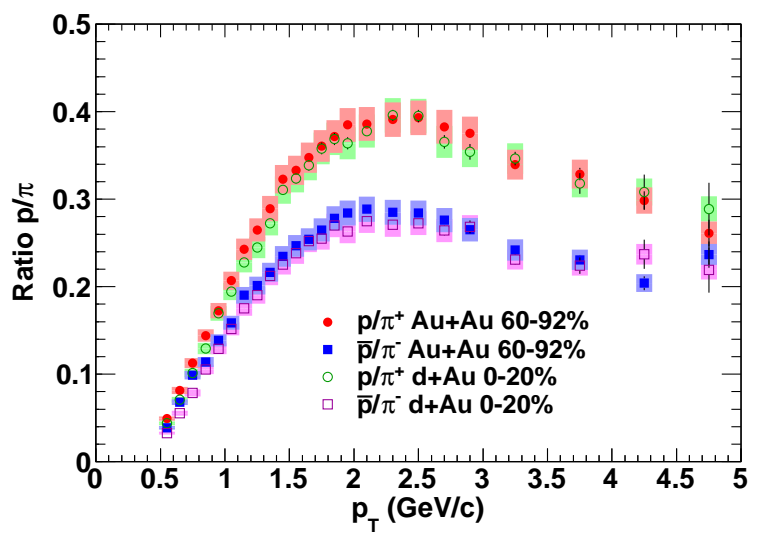

FIG. 14: (Color online) Ratio of $p / \pi^{+}$and $\bar{p} / \pi^{-}$as a function of $p_{T}$ in peripheral $\mathrm{Au}+\mathrm{Au}$ and central $d+\mathrm{Au}$ collisions plotted together. 
apparent in the $R_{d A}$ is that the enhancement for protons begins to fall off at $3.0 \mathrm{GeV} / c$ and steadily drops with increasing $p_{T}$, appearing nearly unmodified at the highest $p_{T}$ points.

The $R_{d A}$ of $\pi, K, \phi$, and $p$ show significant dependence on the number of valence quarks, and no dependence on particle mass. That the baryon $R_{d A}$ is quite different from that of the mesons suggests that recombination plays a role in particle production in $d+\mathrm{Au}$ collisions as well as $\mathrm{Au}+\mathrm{Au}$. The kaon $R_{d A}$ is consistent with the pion $R_{d A}$, in contrast to $R_{\mathrm{AA}}$ where the kaons are consistently above the pions. This is consistent with the $K / \pi$ ratio discussed above and indicates that there is no discernible strangeness enhancement within uncertainties in $d+\mathrm{Au}$ collisions.

\section{Comparison of Peripheral $\mathrm{Au}+\mathrm{Au}$ to Central $d+\mathbf{A u}$}

Motivated by the remarkable similarities between peripheral $\mathrm{Au}+\mathrm{Au}$ and central $d+\mathrm{Au}$ collisions, we now compare the two directly. Figure 13 shows the $K / \pi$ ratio and Fig. 14 shows the $p / \pi$ ratio in peripheral $\mathrm{Au}+\mathrm{Au}$ and central $d+\mathrm{Au}$ collisions plotted together. In both cases the ratios are completely consistent with each other between the different collision species, suggesting that the particle production mechanisms in peripheral $\mathrm{Au}+\mathrm{Au}$ and central $d+\mathrm{Au}$ collisions are quite similar.

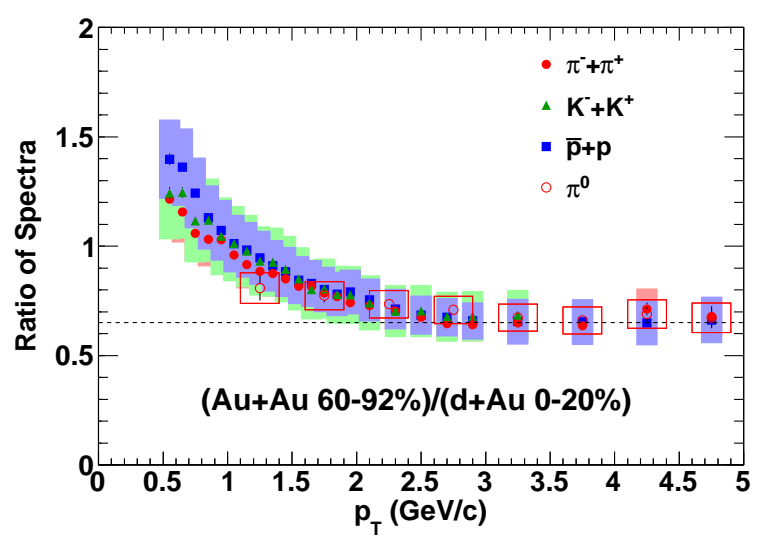

FIG. 15: (Color online) Ratio of invariant yield of particles in peripheral $\mathrm{Au}+\mathrm{Au}(60-92 \%)$ to central $d+\mathrm{Au}(0-20 \%)$ collisions as a function of $p_{T}$.

Figure 15 shows the ratio of the spectra in peripheral $\mathrm{Au}+\mathrm{Au}$ to central $d+\mathrm{Au}$ collisions for pions, kaons, and protons. Also plotted is the ratio for neutral pions, as determined from the data in Refs. [54, 55]. The ratio for $\pi^{0}$ shows excellent agreement with the $\pi^{ \pm}$ratios.

We note that $N_{\text {coll }}$ values are within $2 \%$ of each other and that the $N_{\text {part }}$ values are within $4 \%$ of each other. Both of these differences are well within the associated systematic uncertainties of the Glauber MC values. No scaling is applied, but a scaling by the respective $N_{\text {coll }}$ or
$N_{\text {part }}$ values would change the data imperceptibly. The ratios tend to the same value of roughly 0.65 for each particle species at and above $2.5-3 \mathrm{GeV} / c$. This universal scaling is strongly suggestive of a common particle production mechanism between peripheral $\mathrm{Au}+\mathrm{Au}$ and central $d+\mathrm{Au}$ collisions. It is also interesting to observe that at the lower $p_{T}$, where this ratio rises strongly, there is minimal mass or particle type dependence.

Given the fact that both $N_{\text {coll }}$ and $N_{\text {part }}$ are essentially the same in these two systems, any quantity or physical effect that scales with either of these should be the same in each system, and thus should cancel almost exactly in this ratio. If we make the simple assumption that most or all of the cold nuclear matter effects scale with $N_{\text {coll }}$ or $N_{\text {part }}$, then those effects are completely canceled in this ratio, leaving only the hot nuclear matter effects present in the peripheral $\mathrm{Au}+\mathrm{Au}$ collisions. This could mean that this ratio being less than unity is attributable to the parton energy loss in peripheral $\mathrm{Au}+\mathrm{Au}$. This picture is consistent with the findings in this paper and elsewhere that the $R_{\mathrm{AA}}$ of mesons indicates parton energy loss in the medium even in peripheral $\mathrm{Au}+\mathrm{Au}$ collisions. It is striking, then, that this ratio is independent of particle species, which is suggestive of similar energy loss effects even for protons. This indicates that the baryon enhancement mechanism is the same in both systems.

However, although $N_{\text {coll }}$ and $N_{\text {part }}$ are consistent for the two systems, there is an inherent participant asymmetry that needs to be taken into account. In the case of peripheral $\mathrm{Au}+\mathrm{Au}$ collisions, one has a scenario in which 7 or 8 nucleons on the edge of one $\mathrm{Au}$ nucleus collide against 7 or 8 nucleons on the edge of the other $\mathrm{Au} n u-$ cleus. On the other hand, in the case of central $d+\mathrm{Au}$ collisions, one has a scenario in which the 2 nucleons of the deuteron collide against 13 nucleons in the center of the $\mathrm{Au}$ nucleus. This introduces several additional factors that need to be considered. For example, the participant asymmetry produces a rapidity shift in the particle production [59]. This may explain a deficit of soft particles at low $p_{T}$ in $d+\mathrm{Au}$ collisions at midrapidity, which in turn would explain why the ratio trends up at low $p_{T}$. We also note this low $p_{T}$ region where the ratio rises is where hydrodynamics effects are known to be important in $\mathrm{Au}+\mathrm{Au}$ collisions. It is possible that there are collective flow effects in $d+\mathrm{Au}$ collisions as well, as suggested by the recent results reported in Refs. [26 29]. A full viscous hydrodynamics model comparison is warranted.

Another issue to consider is the modification of parton distribution functions (PDFs) in nuclei. These nuclear PDFs (nPDFs) are known to be modified from the PDFs of single nucleons [7, 60]. The experimentally measured nPDFs are averaged over the entire nucleus and are typically compared to the deuteron PDF to determine the nuclear modification. The binary collisions in peripheral $\mathrm{Au}+\mathrm{Au}$ involve two nucleons which have modified nPDFs. On the other hand, the binary collisions in central $d+\mathrm{Au}$ involve an approximately unmodified nucleon from the deuteron and a modified nucleon from the 
$\mathrm{Au}$ nucleus. Physical observables sensitive to the nPDFs would then be expected to be different for the two systems. However, it is possible that the nucleons in the more diffuse outer region of the nucleus have a different modification from those in the denser center. Therefore, it is not possible to make any model-independent quantitative statements about the differences between the nPDFs in these two systems.

\section{SUMMARY}

In summary we present a highly detailed and systematic study of identified charged hadron spectra and ratios as a function of $p_{T}$ and centrality for $\mathrm{Au}+\mathrm{Au}$ and $d+\mathrm{Au}$ collisions at $\sqrt{s_{N N}} 200 \mathrm{GeV}$. As has been reported previously, we find a baryon enhancement present in both systems. In $d+\mathrm{Au}$ collisions, the Cronin enhancement has long been known to be stronger for baryons than for mesons. However, for the first time a study with enough statistical and systematic precision presents clear evidence for a strong centrality dependence of this effect. In $\mathrm{Au}+\mathrm{Au}$ collisions the baryon enhancement has been attributed to parton recombination as the mode of hadronization. A version of the recombination model has been applied to $d+\mathrm{Au}$ collisions as well, which reproduces the baryon vs. meson differences. The present data strongly suggest that further theoretical investigation is warranted. Given the excellent statistical precision of the present data set, a direct comparison between the two is made for the first time. Specifically, a ratio of the spectra in the most peripheral $\mathrm{Au}+\mathrm{Au}$ and most central $d+\mathrm{Au}$ collisions is measured. These two systems have nearly identical values of both $N_{\text {coll }}$ and $N_{\text {part }}$. Therefore, a direct comparison between the two cancels out a large number of physical effects. We conclude that the baryon enhancement present in both systems is likely driven by a common hadronization mechanism.

\section{Acknowledgments}

We thank the staff of the Collider-Accelerator and Physics Departments at Brookhaven National Laboratory and the staff of the other PHENIX participating institutions for their vital contributions. We acknowledge support from the Office of Nuclear Physics in the Office of Science of the Department of Energy, the National Science Foundation, Abilene Christian University Research Council, Research Foundation of SUNY, and Dean of the College of Arts and Sciences, Vanderbilt University (U.S.A), Ministry of Education, Culture, Sports, Science, and Technology and the Japan Society for the Promotion of Science (Japan), Conselho Nacional de Desenvolvimento Científico e Tecnológico and Fundação de Amparo à Pesquisa do Estado de São Paulo (Brazil), Natural Science Foundation of China (P. R. China), Ministry of Education, Youth and Sports (Czech Republic), Centre National de la Recherche Scientifique, Commissariat à l'Énergie Atomique, and Institut National de Physique Nucléaire et de Physique des Particules (France), Bundesministerium für Bildung und Forschung, Deutscher Akademischer Austausch Dienst, and Alexander von Humboldt Stiftung (Germany), Hungarian National Science Fund, OTKA (Hungary), Department of Atomic Energy and Department of Science and Technology (India), Israel Science Foundation (Israel), National Research Foundation and WCU program of the Ministry Education Science and Technology (Korea), Ministry of Education and Science, Russian Academy of Sciences, Federal Agency of Atomic Energy (Russia), VR and Wallenberg Foundation (Sweden), the U.S. Civilian Research and Development Foundation for the Independent States of the Former Soviet Union, the US-Hungarian Fulbright Foundation for Educational Exchange, and the US-Israel Binational Science Foundation.
[1] K. Adcox et al. (PHENIX Collaboration), Nucl. Phys. A 757, 184 (2005).

[2] J. Adams et al. (STAR Collaboration), Nucl. Phys. A 757, 102 (2005).

[3] B. B. Back et al. (PHOBOS Collaboration), Nucl. Phys. A 757, 28 (2005).

[4] I. Arsene et al. (BRAHMS Collaboration), Nucl. Phys. A 757, 1 (2005).

[5] J. Cronin, H. J. Frisch, M. Shochet, J. Boymond, R. Mermod, et al., Phys. Rev. D 11, 3105 (1975).

[6] D. Antreasyan, J. Cronin, H. J. Frisch, M. Shochet, L. Kluberg, et al., Phys. Rev. D 19, 764 (1979).

[7] M. Arneodo, Phys. Rept. 240, 301 (1994).

[8] E. Iancu and R. Venugopalan, arXiv:0303204 (2003).

[9] S. S. Adler et al. (PHENIX), Phys. Rev. Lett. 91, 172301 (2003).

[10] S. Adler et al. (PHENIX Collaboration), Phys. Rev. C 69, 034909 (2004).
[11] J. Adams et al. (STAR Collaboration), Phys. Rev. Lett. 92, 052302 (2004).

[12] U. Heinz et al., Nucl. Phys. A 702, 269 (2002).

[13] T. Hirano and Y. Nara, Phys. Rev. C 69, 034908 (2004).

[14] I. Vitev and M. Gyulassy, Phys. Rev. C 65, 041902 (2002).

[15] I. Vitev and M. Gyulassy, Nucl. Phys. A 715, 779 (2003).

[16] R. C. Hwa and C. B. Yang, Phys. Rev. C 67, 034902 (2003).

[17] R. J. Fries, B. Muller, C. Nonaka, and S. A. Bass, Phys. Rev. Lett. 90, 202303 (2003).

[18] R. J. Fries, B. Muller, C. Nonaka, and S. A. Bass, Phys. Rev. C 68, 044902 (2003).

[19] V. Greco, C. M. Ko, and P. Levai, Phys. Rev. Lett. 90, $202302(2003)$.

[20] V. Greco, C. M. Ko, and P. Levai, Phys. Rev. C 68, 034904 (2003).

[21] D. Molnar and S. A. Voloshin, Phys. Rev. Lett. 91, 
092301 (2003).

[22] R. C. Hwa and C. B. Yang, Phys. Rev. Lett. 93, 082302 (2004).

[23] R. C. Hwa and C. B. Yang, Phys. Rev. C 70, 037901 (2004).

[24] A. Accardi, arXiv:0212148 (2002).

[25] S. Adler et al. (PHENIX Collaboration), Phys. Rev. C 74, 024904 (2006).

[26] A. Adare et al., (PHENIX Collaboration, arXiv: 1303.1794 (2013).

[27] S. Chatrchyan et al. (CMS Collaboration), Phys. Lett. B 718, 795 (2013).

[28] B. Abelev et al. (ALICE Collaboration), Phys. Lett. B 719, 29 (2013).

[29] G. Aad et al., (ATLAS Collaboration), arXiv: 1212.5198 (2012).

[30] S. A. Bass et al., Nucl. Phys. A 661, 205 (1999).

[31] J. Rafelski and B. Muller, Phys. Rev. Lett. 48, 1066 (1982).

[32] P. Koch and B. Muller, Phys. Rep. 142, 167 (1986).

[33] K. Adcox et al. (PHENIX Collaboration), Nucl. Instrum. Methods A 499, 469 (2003).

[34] K. Ikematsu et al., Nucl. Instrum. Methods A 411, 238 (1998).

[35] M. Allen et al. (PHENIX), Nucl. Instrum. Methods A 499, 549 (2003).

[36] V. G. Ryabov, Nucl. Instrum. Methods A 419, 363 (1998).

[37] J. Barrette et al., Nucl. Phys. A 661, 665 (1999).

[38] B. Love, Masters thesis, Vanderbilt University (2009), URL http://www.phenix.bnl.gov/WWW/talk/archive/theses/

[39] A. Adare et al., (PHENIX Collaboration), arXiv:1203.2644 (2012).

[40] M. L. Miller, K. Reygers, S. J. Sanders, and P. Steinberg, Ann. Rev. Nucl. Part. Sci. 57, 205 (2007).

[41] S. Adler et al. (PHENIX Collaboration), Phys. Rev. Lett. 94, 082302 (2005).
[42] K. Adcox et al. (PHENIX Collaboration), Phys. Rev. Lett. 89, 092302 (2002).

[43] J. Adams et al. (STAR Collaboration), Phys. Rev. Lett. 98, 062301 (2007).

[44] R. Ansorge et al. (UA5 Collaboration), Nucl. Phys. B 328, 36 (1989).

[45] B. Hippolyte, (STAR Collaboration), nucl-ex/0306017 (2003).

[46] A. Billmeier (STAR Collaboration), J. Phys. G 30, S363 (2004)

[47] A. Adare et al. (PHENIX Collaboration), Phys. Rev. C 83, 064903 (2011).

[48] G. Agakishiev et al. (STAR Collaboration), Phys. Rev. Lett. 108, 072302 (2012).

[49] X.-N. Wang, Phys. Rev. C 58, 2321 (1998).

[50] M. Kaneta and N. Xu, nucl-th/0405068 (2004).

[51] J. Cleymans, B. Kampfer, M. Kaneta, S. Wheaton, and N. Xu, Phys. Rev. C 71, 054901 (2005).

[52] A. Adare et al. (PHENIX Collaboration), Phys. Rev. C 83, 024909 (2011).

[53] R. C. Hwa and C. B. Yang, Phys. Rev. C 75, 054904 (2007).

[54] A. Adare et al. (PHENIX Collaboration), Phys. Rev. Lett. 101, 232301 (2008).

[55] S. Adler et al. (PHENIX Collaboration), Phys. Rev. Lett. 98, 172302 (2007).

[56] B. Abelev et al. (STAR Collaboration), Phys. Rev. Lett. 97, 152301 (2006).

[57] S. Adler et al. (PHENIX Collaboration), Phys. Rev. C 76, 034904 (2007).

[58] S. Adler et al. (PHENIX Collaboration), Phys. Rev Lett.

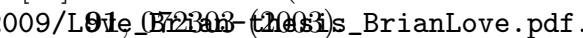

[59] B. Back et al. (PHOBOS Collaboration), Phys. Rev. Lett. 93, 082301 (2004).

[60] K. Eskola, H. Paukkunen, and C. Salgado, JHEP 0904, 065 (2009). 